University of Rhode Island

DigitalCommons@URI

Open Access Dissertations

2012

\title{
Quantifying the Effects of Tidal Restrictions and Plant Invasions on Resident Fish in Atlantic Coast Salt Marshes
}

Kimberly Lellis Dibble

University of Rhode Island, klellisdibble@gmail.com

Follow this and additional works at: https://digitalcommons.uri.edu/oa_diss

\section{Recommended Citation}

Dibble, Kimberly Lellis, "Quantifying the Effects of Tidal Restrictions and Plant Invasions on Resident Fish in Atlantic Coast Salt Marshes" (2012). Open Access Dissertations. Paper 103.

https://digitalcommons.uri.edu/oa_diss/103

This Dissertation is brought to you for free and open access by DigitalCommons@URI. It has been accepted for inclusion in Open Access Dissertations by an authorized administrator of DigitalCommons@URI. For more information, please contact digitalcommons-group@uri.edu. 
QUANTIFYING THE EFFECTS OF TIDAL RESTRICTIONS AND

PLANT INVASIONS ON RESIDENT FISH IN

ATLANTIC COAST SALT MARSHES

BY

KIMBERLY LELLIS DIBBLE

A DISSERTATION SUBMITTED IN PARTIAL FULFILLMENT OF THE

REQUIREMENTS FOR THE DEGREE OF

DOCTOR OF PHILOSOPHY

IN

BIOLOGICAL AND ENVIRONMENTAL SCIENCES

UNIVERSITY OF RHODE ISLAND

2012 


\title{
DOCTOR OF PHILOSOPHY DISSERTATION
}

OF

\author{
KIMBERLY LELLIS DIBBLE
}

\section{APPROVED:}

Dissertation Committee:

$\begin{array}{ll}\text { Major Professor } & \text { Laura A. Meyerson } \\ & \text { Scott R. McWilliams } \\ & \text { Marta Gomez-Chiarri } \\ & \text { Nasser H. Zawia } \\ & \text { DEAN OF THE GRADUATE SCHOOL }\end{array}$




\begin{abstract}
This research investigates the combined effects of tidal restriction and Phragmites australis invasion on habitat quality for marsh fauna across a large geographic area while evaluating the reversal of those effects through ecological restoration. We used meta-analytic tools and published literature to examine differences in the quantity, condition, and diversity of fauna in invaded and restored marshes relative to uninvaded (reference) marshes in the mid-Atlantic and in New England. In addition, we collected data for two projects designed to move beyond the collection of community data (e.g., density, richness, as included in the meta-analysis) to assess the functional response of nekton to tidal restrictions and habitat restoration.

We examined community data from 43 published studies that compared faunal patterns in $P$. australis vs. native Spartina alterniflora marshes. Using the log response ratio, we found a decrease in the quantity and condition of fauna in invaded marshes relative to reference marshes. We detected differences by region, habitat type, taxonomic group, and life history stage, with adverse impacts to fauna residing in the mid-Atlantic, to those utilizing the marsh surface, for nekton, and particularly for the larval/juvenile life history stage. We compared data from restored and reference marshes and found no significant differences across all categorical variables, suggesting that impacts of the $P$. australis invasion were reversed through restoration. We examined impacts to the functional role of salt marshes by assessing how the change in dominant primary producer from native $S$. alterniflora to introduced $P$. australis affects energy flow through salt marsh food webs. We found that palatable dietary items such as suspended particulate matter (SPM, a proxy for phytoplankton)
\end{abstract}


and benthic microalgae (BMA) are important primary producers at the food web base in reference marshes for Fundulus heteroclitus, a resident secondary consumer. In restricted marshes primary consumers rely on SPM and less on BMA, resulting in a shift in diet toward invasive plant consumption. This is likely due to increased shading of the marsh surface that decreases BMA biomass, which has also been noted in midAtlantic marshes. Restoration increased the importance of BMA, indicating a shift in ecological recovery toward the uninvaded state.

Using physiological and morphological indicators of fish condition, we found that $F$. heteroclitus in restricted marshes exhibit significant reductions in energy reserves, lower proportions of gravid females, and higher incidences of parasitism relative to fish in unrestricted salt marshes. Parasitized fish exhibit significant reductions in lipid reserves; however, when parasitized individuals were removed from the analysis the significant difference between the restricted and reference marsh fish remained. Fish in tidally restored marshes were equivalent to those in unrestricted marshes, with similar energy reserves, gravidity, and parasite load. Fish in all marshes (regardless of restriction status) exhibited similar growth rates and morphology.

Overall, results indicate that tidal restrictions and subsequent $P$. australis invasion has reduced the quality of habitat for estuarine communities including the dominant salt marsh resident, $F$. heteroclitus. However, our analyses indicate that ecological restoration can mitigate these effects over relatively short time scales. These findings should be of great interest to restoration practitioners, particularly those that are currently making habitat management decisions regarding the restoration of coastal salt marshes colonized by common reed. 


\section{ACKNOWLEDGEMENTS}

I would like to thank my major professor, Dr. Laura Meyerson, who has helped shape me into the scientist I am today. I appreciate the many long hours you spent reviewing manuscripts and grant proposals, your thoughtful guidance on research methods, for use of your facilities and research equipment, and for freely letting me aromatize your laboratory with the scent of dried, pulverized fish material. I assure you the smell will diminish...some day. I would also like to thank my dissertation committee members, Drs. Scott McWilliams, Marta Gomez-Chiarri, Graham Forrester, and Charley Roman. I appreciate the many hours you invested to craft my research, your input during committee meetings, review of manuscripts and proposals, and for access to your laboratories and equipment that was essential to my research. Many thanks to Deb Bourassa and Judy Palmer, whose work is often overlooked but is essential to the functioning of our research programs and department.

This research would not have been possible without the support of the following agencies and organizations: Environmental Protection Agency Science To Achieve Results Graduate Fellowship, National Oceanic and Atmospheric Administration National Estuarine Research Reserve Graduate Fellowship, National Science Foundation Integrative Graduate Education and Research Traineeship Grant to the Coastal Institute at the University of Rhode Island, Philanthropic Educational Organization, Northeast Aquatic Plant Management Society, Rhode Island Natural History Survey, The Nature Conservancy of Rhode Island, and the URI Coastal Fellows Program, Research Office, Graduate School, Vice Provost's Office, and the Natural Resources Science Department. I thank the federal and state permitting 
agencies that allowed me to do research in their salt marshes. I appreciate housing assistance from the Wells NERR and NPS during our field visits.

I am grateful for the hard work and dedication of the small army of undergraduate and post-graduate students who assisted me with collecting and processing samples, specifically Tiffany Lewis, Alissa Becker, Keri Dyer, and Stephanie Batters. I know that you will never look at a coffee grinder in quite the same way again, but the many hours you spent grinding fish, searching for otoliths the size of a pin head, packing thimbles, and picking small parasites out of body cavities with tweezers has paid off with really amazing data. I hope that I have been able to inspire you as much as you have inspired me. Thank you for keeping me organized and for dealing with my crankiness at 4am as we rushed to sites to catch the incoming tide.

I thank my current and former colleagues, including Penelope Pooler, Megan Tyrrell, Michele Dionne, Rick McKinney, and Tom Bigford. Your guidance and support has greatly influenced my dissertation direction and my research interests as I move forward on this career path. I especially thank my IGERT mentors, including Peter August, Judith Swift, Q Kellogg, Cheryl Foster, Art Gold, Candace Oviatt, Stan Cobb, and Jim Opaluch. You have enriched the lives of all CIIP trainees and you have helped me to find my 'voice' as a future leader in my field. I thank my colleagues from the CIIP cohorts for their advice, friendship, passion, and inspiration, specifically Carrie Byron, Nicole Rohr, and Nate Vinhateiro. "Its About a Feeling" resonates with me still and I know we will continue to be each other's soundboards for new ideas and perhaps future collaborations. 
Many thanks to my immediate and extended families who have supported me throughout this journey. I thank my sister for lending her ear and for the wonderful care packages. My parents have encouraged me throughout life to work hard and always go for the things once thought unobtainable. I thank you for your support and for not telling me I was crazy to give up my policy career to pursue this goal. I thank you immensely for being my fall field assistants. Not many people have parents that will take weeks off to slog through salt marshes, get attacked by swarms of mosquitoes, work from sunup to sundown, decapitate fish, pick parasites, and race against the clock to get everything done in time. These are the moments I treasure the most—seeing Mom in her 'chugging gear, paddling across the marsh with her prized trap full of fish in a Maine salt marsh. And Dad, long and lanky bravely sent into the depths of the Hatches Harbor incoming current to extract the last bit of water quality data. Thank you for always reminding me to have fun with life.

I thank my dog Bailey, who has slept patiently by my side while writing this dissertation. We can now resume our daily walks. Thank you for busting through Phragmites to clear a path for me in the field, and for not eating my samples even though they looked tasty. And last, but certainly not least, I thank my husband Nathan, who inspired and encouraged me to get out of my cubicle in concrete world and back into the field that I am most passionate about. You have been my biggest cheerleader, my supporter, my in-house graphic designer, my most animated field assistant, and my anchor throughout this whole process. Thank you for encouraging me to push on even after that first awful day in the field. I could not have finished this degree without you. Life is truly an adventure, and I look forward to taking the next big leaps with you. 


\section{PREFACE}

This dissertation is written in manuscript format with four main chapters each comprising a separate manuscript. The first chapter, Impacts of plant invasions can be reversed through restoration: A regional meta-analysis of faunal communities, follows the manuscript formatting requirements of the journal Biological Invasions and is currently in revision. The second chapter, The importance of an invasive plant in salt marsh food webs, follows the formatting guidelines of the Journal of Ecology and was submitted in August 2012. The third chapter, Tidal flushing restores the physiological condition of fish residing in degraded salt marshes, follows the manuscript formatting requirements of the journal PLoS ONE and was published in September 2012. The

fourth chapter, Tidal restriction may reduce female fish gravidity in salt marshes, follows the formatting guidelines of the journal Marine Ecology Progress Series and is currently in revision. 


\section{TABLE OF CONTENTS}

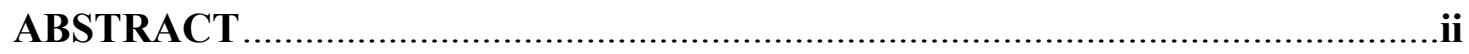

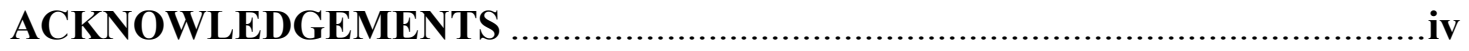

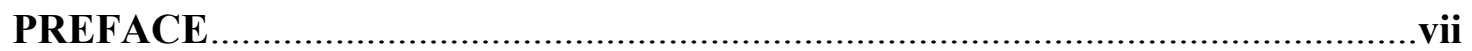

TABLE OF CONTENTS .......................................................................... viii

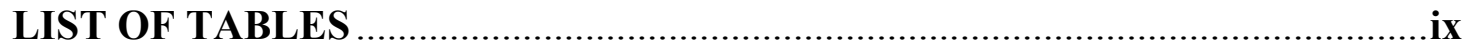

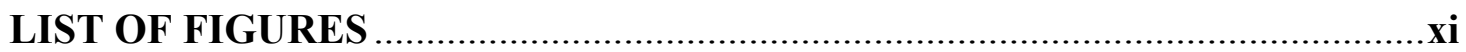

CHAPTER 1: IMPACTS OF PLANT INVASIONS CAN BE REVERSED THROUGH RESTORATION: A REGIONAL META-ANALYSIS OF

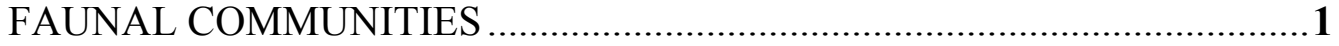

CHAPTER 2: THE IMPORTANCE OF AN INVASIVE PLANT IN SALT MARSH FOOD WEBS .56

CHAPTER 3: TIDAL FLUSHING RESTORES THE PHYSIOLOGICAL CONDITION OF FISH RESIDING IN DEGRADED SALT MARSHES.

CHAPTER 4: TIDAL RESTRICTION MAY REDUCE FEMALE FISH GRAVIDITY IN SALT MARSHES 


\section{LIST OF TABLES}

TABLE

PAGE

\section{CHAPTER 1}

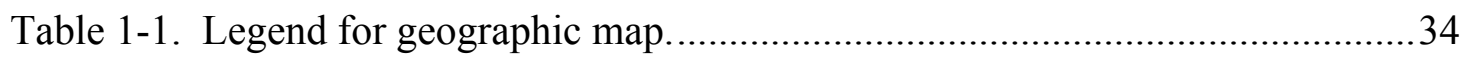

Table 1-2. Response variables included in the invaded and restored meta-analyses...35

Table 1-3. Number of studies in which the invertebrate prey exhibited higher raw abundances in Spartina spp. or P. australis habitat....................................36

Table 1-A1. Studies included in the meta-analysis..........................................45

Table 1-A2. Stable isotope studies reviewed, with their primary conclusion. ............50

Table 1-A3. Summary of data collection approach for meta-analysis. ......................53

Table 1-A4. Criteria used to minimize bias caused by non-independence of data and to group data by response variable.

\section{CHAPTER 2}

Table 2-1. Summary of data for primary producers, fish, and eggs by marsh type.....90

Table 2-2. Discrimination factors used in sensitivity analysis with model output.....91

\section{CHAPTER 3}

Table 3-1. Characteristics of our New England study sites.

Table 3-2. Mean water quality 2010-2011, by marsh type. 136

Table 3-3. Parasites infecting Fundulus heteroclitus by marsh type, 2010-2011.....137

Table 3-4. Mean proximate body composition of fish in study, 2010-2011. 138 
Table 3-5. Results of repeated measures ANOVA for the restricted vs. reference systems.

Table 3-6. Mean otolith measurements for fish in study, 2010-2011. .140

\section{CHAPTER 4}

Table 4-1. Mean water quality measurements.

Table 4-2. Mean fish length, range (in parentheses), and sample size of female $F$.

heteroclitus. 168

Table 4-3. Logistic regression using fish length as a covariate.

Table 4-4. Mean fish wet mass, range (in parentheses), and sample size of female $F$.

$$
\text { heteroclitus...... }
$$

Table 4-5. Logistic regression using fish wet mass as a covariate. 


\section{LIST OF FIGURES}

FIGURE

PAGE

\section{CHAPTER 1}

Figure 1-1. Geographic locations of authors' study sites. 37

Figure 1-2. Number of studies in the literature concluding that introduced P. australis has negative, positive, or no effect (neutral) on habitat quality for salt marsh biota at invaded and restored sites.

Figure 1-3. Results of meta-analysis by the 'metric' categorical variable.

Figure 1-4. Regional differences in effect size on fauna residing in restored and invaded systems.

Figure 1-5. Regional differences by response metric in invaded and restored wetlands.

Figure 1-6. Meta-analysis results by taxon and life history stage.

Figure 1-7. Meta-analysis results by marsh habitat and system type.

Figure 1-8. Differences in faunal patterns in hydrologically vs. conventionally restored sites.

\section{CHAPTER 2}

Figure 2-1. Map of study site locations.

Figure 2-2. Carbon stable isotope composition of suspended particulate matter (SPM) and dissolved inorganic carbon (DI) vs. water column salinity for all study sites. 
Figure 2-3. Relationship between the carbon isotopic compositions of suspended particulate matter (SPM) and dissolved inorganic carbon (DI) in the water column for all study sites.

Figure 2-4. Stable isotope biplots for (a) $\delta^{13} \mathrm{C}$ vs. $\delta^{15} \mathrm{~N}$ and (b) $\delta^{13} \mathrm{C}$ vs. $\delta^{34} \mathrm{~S}$ for the restricted vs. reference (control) marsh sites.

Figure 2-5. Stable isotope biplots for (a) $\delta^{13} \mathrm{C}$ vs. $\delta^{15} \mathrm{~N}$ and (b) $\delta^{13} \mathrm{C}$ vs. $\delta^{34} \mathrm{~S}$ for the restored vs. reference (control) marsh sites.

\section{CHAPTER 3}

Figure 3-1. Map of study site locations in New England. 141

Figure 3-2. Proportion of fish parasitized (circles; females and males) or gravid

(triangles; females only) by marsh type.

Figure 3-3. Proximate body composition of fish. 143

Figure 3-4. Number of fish captured by age group and marsh type.

Figure 3-5. Fish length vs. otolith radius for healthy fish. 145

Figure 3-6. Fish length vs. wet weight for healthy fish. 146

\section{CHAPTER 4}

Figure 4-1. Study site locations in New England.

Figure 4-2. Proportion of gravid female fish in each marsh type, July 2011. 173 


\section{CHAPTER 1}

\section{Impacts of plant invasions can be reversed through restoration:}

\section{A regional meta-analysis of faunal communities}

by

Kimberly L. Dibble ${ }^{1}$; Penelope S. Pooler ${ }^{2}$; Laura A. Meyerson ${ }^{1,3}$

is in revision at Biological Invasions

${ }^{1}$ Department of Natural Resources Science, University of Rhode Island, 1 Greenhouse Road, Kingston, RI 02881.

${ }^{2}$ National Park Service, Northeast Coastal and Barrier Network, University of Rhode Island, 1 Greenhouse Road, Kingston, RI 02881.

${ }^{3}$ Institute of Botany, Department of Invasion Ecology, Academy of Sciences of the Czech Republic, Průhonice, CZ 252 43, Czech Republic. 


\begin{abstract}
We conducted a meta-analysis of empirical evidence on the effects of a widespread invasive macrophyte (Phragmites australis) on faunal communities by comparing invaded to uninvaded marshes and by evaluating the reversal of those effects through ecological restoration. Relative to uninvaded Spartina alterniflora marshes, the quantity and condition of fauna residing in invaded marshes was significantly poorer. We detected adverse impacts to fauna residing in the mid-Atlantic but not in the New England region and to fauna utilizing the marsh surface but not to those inhabiting tidal creeks. By taxonomic group, we found that the invasion negatively affected nekton but not invertebrates. Both adult and sub-adult organisms were adversely affected, although the magnitude of the effect on the sub-adult life history stage was four times larger than that for adults. When restored marshes were compared to reference marshes, there were no significant differences across all categorical variables suggesting that the negative impacts of the invasion were reversed through restoration. A separate qualitative review of trophic data indicated that benthic microalgae and the dominant macrophyte were important primary producers at the base of the food web in reference, restored, and invaded salt marshes. The relative importance to diet and overall quantity of microalgae decreases in highly invaded systems due to decreased light, which could affect energy flow through the food web. Both analyses revealed that while estuarine communities are adversely affected by $P$. australis, long-term effects may be mitigated by restoration over relatively short time scales.
\end{abstract}


Keywords: non-native; salt marsh; Phragmites; Spartina; log response ratio; bootstrapping.

\section{Introduction}

Several regional and globally focused qualitative reviews and meta-analyses have synthesized published data on invasive plant traits, the effectiveness of control measures, and impacts to native plant biodiversity (Davidson et al. 2011; Kettenring and Reinhardt Adams 2011; Powell et al. 2011). However, few meta-analyses have synthesized the impacts of invasive plants on fauna, and those that have provide only a global "snapshot" of multiple effects of several invasive plant species on resident animal communities (Vilà et al. 2011; Pyšek et al. 2012). We conducted a regional meta-analysis using data mined from the literature to quantify the ecological effects of a globally common plant, Phragmites australis, on the quantity, condition, and diversity of faunal communities residing in invaded habitats along the Atlantic coast of North America. We then conducted a second meta-analysis to test whether manipulations to remove the invasive plant successfully restored the faunal community. Finally, we reviewed available food web data to determine how a change in the dominant plant community (from Spartina alterniflora to $P$. australis) affects energy flow to fauna at higher trophic levels.

Phragmites australis is a globally distributed macrophyte that colonizes a wide range of environmental conditions, successfully invades new habitats, and has been targeted for eradication in North America (Roman et al. 1984; Chambers et al. 1999; 
Farnsworth and Meyerson 2003). The non-native strain was likely introduced multiple times over the last two centuries and has since spread throughout New England, the mid-Atlantic, and elsewhere in North America (Saltonstall 2002; Kulmatiski et al. 2010; Hauber et al. 2011; Lambertini et al. 2012). Introduced P. australis colonizes disturbed marshes and is particularly competitive under eutrophic conditions (Roman et al. 1984; Meyerson et al. 2000; Mozder and Zieman 2010).

This invader transforms the estuarine landscape by replacing short-statured grasses with a dense monoculture of tall reed grass that reduces native plant diversity, light, temperature, and nutrient cycling (Meyerson et al. 1999, 2000; Windham and Meyerson 2003). Introduced $P$. australis facilitates marsh accretion via a thick aboveground biomass mat of living and slowly decomposing organic matter that traps mineral and organic sediment (Rooth et al. 2003). Marsh accretion and structural impediments caused by $P$. australis colonization can reduce bird nesting habitat, limit nekton access to intertidal habitats used for feeding, refuge, and reproduction, and alter energy flow from herbivores to higher trophic levels in the invaded range (Benoit and Askins 1999; Able et al. 2003; Gratton and Denno 2006).

Introduced $P$. australis continues to expand its range in North America, which lends importance to gaining a better understanding of the effects of this invasion. Because evidence in the literature is equivocal on the impact to fauna resulting from this invasion (Weinstein et al. 2003; Weis and Butler 2009), we asked the following questions: a) does the replacement of native plant communities by an invasive plant 
alter the quantity, condition, or diversity of faunal communities?; b) does the magnitude and direction of faunal effects vary geographically, spatially, taxonomically, or by life history stage?; and c) can restoration of the native plant community reverse the negative effects of invasive plants on fauna?

\section{Methods}

In this paper, we conducted two reviews. First, we used meta-analysis to quantitatively synthesize data from 43 publications on faunal quantity, condition, and diversity (Online Resource 1, Table 1-A1). Second, to examine the contribution of macrophytes to the food web, we qualitatively reviewed 11 published stable isotope studies (Online Resource 1, Table 1-A2) that could not be compared quantitatively because naturally occurring isotopes of nitrogen and sulfur fluctuate due to local environmental conditions and sample methodology (species trophic level, tissue sampled; Fry 2006), which differed among studies.

\section{Terminology}

We restricted our meta-analysis to studies that compared faunal impacts within a treatment marsh to a control marsh within the same publication. The treatment represented effects on fauna due to habitat invasion (by $P$. australis) or habitat restoration (removal of $P$. australis by tidal/physical/chemical restoration), whereas the control was a non-invaded, unrestricted reference system adjacent to or near the invaded or restored marshes. Reference marshes were those that would be present if the tidal restriction and invasion had not occurred (i.e., Spartina alterniflora, $S$. 
patens, Distichlis spicata, Juncus gerardii, etc.). We used data from publications with two foci: 1) invasion studies: those that compared fauna in invaded marshes to those in reference marshes; and 2) restoration studies: those that compared fauna in restored marshes to those in reference marshes. We classified a publication as both an invasion and a restoration study if the author presented both pre- and post-restoration data.

\section{Data Selection}

From February to August 2011, we searched electronic databases for germane literature using combinations of selection terms (Online Resource 1, Table 1-A3) and identified $600+$ publications, reports, theses, and dissertations relevant to our topic. We screened each by reading the abstract and narrowed the list to 105 publications that specifically focused on P. australis invaded marshes in North America. We then further narrowed the list to 54 publications (43 meta-analysis, 11 isotope) using the following a priori criteria: 1) the study was conducted along the Atlantic coast of North America, spanning southern Florida to Nova Scotia (Fig. 1-1, Table 1-1); 2) research sites were located in tidally influenced salt marshes (freshwater excluded); 3) the study included both a control and treatment marsh located adjacent to or near each other that exhibited similar environmental characteristics; and 4) faunal assemblages were compared between the control and treatment marshes.

\section{Calculation of Effect Sizes}

We calculated effect sizes using standard meta-analytic procedures (Hedges and Olkin 1985; Hedges et al. 1999; Online Resource 1, Table 1-A4). We primarily used the 
unweighted $\log$ response ratio, $L$, which is the natural log of the ratio of the mean outcome in the treatment group to that in the control group (Hedges et al. 1999). This index provides flexibility in effect size calculation because it does not require knowledge of sample sizes and standard deviations, which are not always reported in empirical studies (Adams et al. 1997). The following equation was used to calculate the natural log of the response ratio:

$$
L=\ln \left(\mathrm{X}_{\mathrm{T}}\right)-\ln \left(\mathrm{X}_{\mathrm{C}}\right)
$$

Where $X_{T}$ is the mean of the within-study treatment variable and $X_{C}$ is the mean of the within-study control variable (i.e., the natural log of the mean quantity of fauna in the invaded marsh minus the natural log of the mean quantity of fauna in the reference marsh). The natural log linearizes the metric and normalizes its sampling distribution. Our meta-analysis primarily contains data using this unweighted metric. Unweighted metrics are appropriate to use in ecological studies because they quantify the proportional change resulting from experimental manipulation and have been used to quantify the effects of contaminants, climate change, and other habitat alterations on native species and ecological systems (McKinley and Johnston 2010; Sorte et al. 2010; Whiteway et al. 2010). Unweighted metrics have also been used to assess the response of biological communities to restoration and invasive plant control (Kettenring and Reinhardt Adams 2011; Moreno-Mateos et al. 2012).

Of the 43 meta-analysis studies, we extracted unweighted data from 35 invasion studies and 23 restoration studies (15 studies overlapped, containing data from invaded, restored, and reference sites, i.e., pre- and post-restoration data comparisons). 
However, since a small subset of the 43 studies contained data on standard deviations and sample sizes ( $\mathrm{n}=17$ invasion studies, $\mathrm{n}=11$ restoration studies), we parametrically weighted the smaller subset of studies using the Hedges' $d$ effect size metric and report the mean overall results from the unweighted $(L)$ and weighted $(d)$ response ratios separately for comparison purposes. We calculated Hedges' $d$ by using the means of the treatment and control groups, the pooled standard deviation within each study, and a correction factor (Hedges and Olkin 1985):

$$
\begin{aligned}
& \text { Hedges' } d=\left[\left(\mathrm{X}_{\mathrm{T}}-\mathrm{X}_{\mathrm{C}}\right) / \mathrm{s}_{\mathrm{p}}\right] * J \\
& \left.J=1-\left[3 / 4\left(\mathrm{n}_{\mathrm{T}}+\mathrm{n}_{\mathrm{C}}-2\right)-1\right)\right] \\
& \mathrm{s}_{\mathrm{p}}=\sqrt{ }\left[\left(\left(\mathrm{n}_{\mathrm{T}}-1\right) \mathrm{s}_{\mathrm{T}}{ }^{2}+\left(\mathrm{n}_{\mathrm{C}}-1\right) \mathrm{s}_{\mathrm{C}}{ }^{2}\right) /\left(\mathrm{n}_{\mathrm{T}}+\mathrm{n}_{\mathrm{C}}-2\right)\right]
\end{aligned}
$$

When calculated, ' $d$ ' is equal to the within-study mean of the treatment group $\left(\mathrm{X}_{\mathrm{T}}\right)$ minus the within-study mean of the control group $\left(\mathrm{X}_{\mathrm{C}}\right)$, divided by the pooled standard deviation $\left(\mathrm{s}_{\mathrm{p}}\right)$, multiplied by a correction factor $(J)$. Sample sizes within each treatment and control group are $\mathrm{n}_{\mathrm{T}}$ and $\mathrm{n}_{\mathrm{C}}$, respectively, and within-study standard deviations for the treatment and control groups are $\mathrm{s}_{\mathrm{T}}$ and $\mathrm{s}_{\mathrm{C}}$.

\section{Data Analysis}

We conducted separate meta-analyses of unweighted effect size data from the invasion and restoration studies to discern overall trends of the invasive plant as well as outcomes post-restoration. We analyzed effect sizes with the boot package in the $\mathrm{R}$ statistical software environment (v. 2.14.1; Davison and Hinkley 1997; Canty and Ripley 2011). Using the boot and boot.ci functions in the boot package we calculated 95\% bias-corrected bootstrap confidence intervals by resampling the mean effect size 
datasets. Each confidence interval estimate was based on 10,000 bootstrap samples (Adams et al. 1997). Bias-corrected bootstrap techniques were used to correct for small sample sizes (Efron 1987; Hesterberg et al. 2005), which occurred in some response variable categories. Effect sizes are reported as 95\% confidence intervals and are graphically represented in forest plots that also show each estimated mean. Mean effect sizes within each category were considered significantly different from zero if the confidence intervals (CI) did not contain zero. We analyzed data from each of the response variables separately (Table 1-2). Descriptions of data used within these categories can be found in Online Resource 1, Tables 1-A3 and 1-A4.

\section{Non-Independence of Data and Publication Bias}

Several effect sizes can be calculated from one publication if the author gathered data on multiple species, over several years, using multiple gear types, etc., but this can result in an overrepresentation of a specific study within a meta-analysis (Gurevitch and Hedges 1999). To ensure the data points that we included were independent, we calculated the mean effect size within each study for each categorical variable (see criteria, Online Resource 1, Table 1-A4) and performed a separate analysis across studies on each variable (Gurevitch and Hedges 1999). In addition, we attempted to minimize potential bias caused by the tendency of authors to only publish significant results by including publications from a range of journals having both low and high impact factors and by including publications that reported data on multiple species with a range of effects (negative, positive, and neutral; Arnqvist and Wooster 1995; Gaertner et al. 2009; Kalies et al. 2010). 


\section{Results}

Data from all papers included in this analysis were collected from 31 research sites spanning Maryland to Maine (Fig. 1-1; Table 1-1). Within the studies conducted in the mid-Atlantic region, researchers collected data at 15 sites, but because many study sites were shared among authors research was conducted 52 times in these marshes. Approximately $50 \%$ of the sampling in the mid-Atlantic region $(n=26)$ was conducted in Delaware Bay. In New England, 16 sites were visited, with less overlap in sampling effort ( $\mathrm{n}=31)$. In the invasion studies, $41 \%$ of authors indicated that $P$. australisinvaded marshes were equivalent to uninvaded (reference) habitats for biota, $12 \%$ suggested the invaded marshes provide better habitat, and $46 \%$ reported negative effects of the plant on fauna (Fig. 1-2). In the restoration studies, nearly all of the authors $(83 \%)$ concluded that restored marshes were equivalent habitats to reference marshes for faunal communities (Fig. 1-2).

\section{Meta-Analysis of Faunal Data}

We extracted 281 effect sizes (unweighted log response ratio, $L$ ) from the invasion studies and 123 from the restoration studies. However, to avoid the issue of nonindependence of data, we took the means within studies, resulting in 89 invaded effect sizes and 74 restored effect sizes in our analysis. Across all invasion studies, the grand mean $L$ was -0.624 , while the mean for the restoration studies was -0.017 . Using Hedges' $d$, we extracted 27 invaded effect sizes and 31 restored effect sizes, with a mean $d$ of -0.654 for the invasion studies and -0.099 for the restoration studies. Therefore, the weighted effect size using study-level sample sizes and standard 
deviations indicates an even greater negative effect of the invasive plant on fauna in both invaded and restored habitats. Since we would have had to exclude $>50 \%$ of our data using Hedges' $d$, we conducted the meta-analysis using only the unweighted log response ratio, which is a conservative yet rigorous approach to analyzing data and drawing conclusions (Adams et al. 1997). We refer to the unweighted $L$ as the 'effect size' for the rest of this paper.

Effects by Faunal Condition, Diversity, and Quantity

We report results for the biotic response metric (condition, diversity, and quantity of organisms) in Figure 1-3. Post-invasion changes in habitat structure negatively affected the condition (CI: $-1.176,-0.312 ; n=20)$ and quantity of fauna (CI: -1.364 , $-0.352 ; n=46)$, but not species diversity (CI: $-0.365,0.035 ; n=23)$. Faunal patterns within restored marshes were equivalent to reference conditions, as measured by animal condition (CI: -0.303, 0.009; $\mathrm{n}=16$ ), species diversity (CI: -0.271, 0.134; $\mathrm{n}=21$ ), and total quantity of fauna (CI: $-0.215,0.344 ; n=37$; Fig. $1-3)$.

\section{Effects by Geographic Region}

We analyzed effect size by region and found significant negative effects on fauna in invaded marshes in the mid-Atlantic (CI: $-1.225,-0.502 ; n=51)$, but not in New England (CI: -0.817, 0.109; n=38; Fig. 1-4). We found no significant difference between restored and reference marsh systems in both mid-Atlantic (CI: -0.117, 0.679; $\mathrm{n}=20$ ) and New England (CI: -0.257, 0.069; n=54; Fig. 1-4) salt marshes. Since we saw significant effects in the mid-Atlantic region and for multiple metrics, we 
analyzed the response data by metric and geographic region sampled. Within the midAtlantic, we found significant negative effects of the altered habitat on animal condition (CI: $-1.500,-0.319 ; n=14)$ and faunal quantity (CI: $-1.787,-0.598 ; n=27)$, but not on species diversity (CI: $-0.446,0.005 ; n=10 ;$ Fig. $1-5$ ). In New England, we found no effects on species diversity (CI: $-0.458,0.151 ; n=13$ ) or faunal quantity (CI: $-1.309,0.420 ; \mathrm{n}=19)$. Due to small sample size we could not reliably calculate confidence intervals for faunal condition in New England, but the mean effect size was $-0.422(\mathrm{n}=6)$. For the restored and reference marsh comparisons, we found no significant effect for any breakdown, mirroring the larger analysis by region and metric (CI: condition/New England: -0.284, 0.023; n=12; diversity/New England: $-0.321,0.179 ; n=17$; quantity/New England: $-0.425,0.172 ; n=25 ;$ quantity/midAtlantic: $-0.097,1.097 ; \mathrm{n}=12$ ). Due to small sample sizes, we could not calculate confidence intervals for the condition/mid-Atlantic or diversity/mid-Atlantic categories, but mean effect sizes were $-0.153(n=4)$ and $-0.058(n=4)$, respectively (Fig. 1-5).

\section{Effects by Taxon and Life History Stage}

We quantified effects by taxonomic group and by life history stage and found significant negative effects of the invaded habitat on nekton (fish and swimming crustaceans; CI: $-1.017,-0.305 ; \mathrm{n}=62$ ) but not invertebrates (marsh surface fauna, benthic infauna; CI: $-0.758,0.072 ; n=22$; Fig. 1-6). We did not have sufficient data to statistically analyze birds in invaded wetlands, but the mean effect size $(-1.870 ; \mathrm{n}=5)$ was relatively large and in the negative direction. Both adult (CI: -0.652, -0.132 ; 
$\mathrm{n}=75)$ and sub-adult (CI: $-2.840,-1.054 ; \mathrm{n}=14)$ life history stages were negatively affected in the invaded habitat (Fig. 1-6). When we compared restored and reference marsh biota, we again found no significant difference between systems for nekton (CI: $-0.136,0.201 ; n=65)$ or for the adult life history stage $(\mathrm{CI}:-0.202,0.104 ; n=67)$. Due to small sample size in the restored marshes, we could not calculate confidence intervals for invertebrates, birds, or for the sub-adult life history stage, but mean effect sizes $(0.050 ; n=3),(-0.375 ; n=6)$, and $(0.335 ; n=7)$, respectively, are clustered around the neutral/low effect zone (Fig. 1-6).

\section{Effects by Marsh Habitat and System Type}

We analyzed data by marsh habitat (marsh surface, tidal creek) and system type (i.e., invasion of an open system or behind a tidal restriction) and found that fauna using the marsh surface in invaded marshes were negatively affected (CI: $-1.119,-0.464 ; n=66$ ), whereas those sampled in tidal creeks were unaffected (CI: -0.805, 0.393; n=23; Fig. 1-7). Fauna in invaded marshes open to tidal flow were negatively affected (CI: $-1.008,-0.436 ; n=71)$, whereas those residing in tidally restricted marshes were comparable to those in reference areas (CI: $-1.202,0.489 ; \mathrm{n}=18)$. We found no significant difference between restored and reference marsh fauna using the marsh surface (CI: $-0.165,0.235 ; n=40)$, tidal creeks (CI: $-0.272,0.213 ; n=34)$, sites open to tidal flow (CI: $-0.171,0.537 ; \mathrm{n}=23$ ), and sites where tidal flow was re-established under a road/culvert restriction (CI: -0.244, 0.091; n=51; Fig. 1-7). 


\section{Effects by Restoration Type}

Last, we analyzed effect size for the restoration studies to determine whether the type of restoration (i.e., increased tidal flushing or chemical/physical/mechanical removal) was important in the determination of restoration success. We found no significant difference in faunal abundance patterns between restored and reference systems that were subjected to hydrologic $(\mathrm{CI}:-0.255,0.078 ; \mathrm{n}=52)$ or conventional $(\mathrm{CI}:-0.131$, $0.624 ; \mathrm{n}=22$ ) restoration techniques (Fig. $1-8$ ).

\section{Discussion}

\section{Linkages within Salt Marshes: A Landscape Level Perspective}

Prey Base and Trophic Support

This study is the first to comprehensively assess of the impact of the P. australis plant invasion on salt marsh fauna across a wide geographic range in North America. The Atlantic coast salt marsh landscape is intricately linked, with energy flowing from primary producers via invertebrates to nekton and birds (Peterson 1999; Brittain et al. 2012). Benthic microalgae represent an important food source for invertebrate prey at the base of the food web, likely due to its high palatability in comparison to vascular plants (Deegan and Garritt 1997; Currin et al. 2003). The quantity of benthic microalgae available for trophic support can be diminished in highly invaded $P$. australis marshes due to enhanced shading of the marsh surface, which could affect energy flow from the prey base to upper trophic levels (see review in Online Resource 1, Table 1-A2). 
Consumption of invertebrate prey dominates the diets of higher trophic level organisms in salt marsh ecosystems (James-Pirri et al. 2001; Brittain et al. 2012). Gratton and Denno (2005) investigated post-invasion changes in invertebrate prey base and found evidence that arthropod community assemblages shifted from those dominated by external free-living specialists (e.g., spiders, predators, free-living chewers) in natural marshes to concealed detritivorous chewers in introduced $P$. australis. Stable isotope data confirmed a shift in trophic support, as arthropods in reference marshes consumed Spartina spp. and those in P. australis relied on detrital or algal food sources and not the dominant macrophyte (Gratton and Denno 2006). This represents both a loss of nutrient export off the marsh surface and a shift toward concealed invertebrate communities that may be less accessible to consumers.

Our meta-analysis did not detect overall negative effects of the plant invader on invertebrates but this may be due to methods used to ensure the non-independence of our data. By taking the mean abundance of species within each taxonomic group in the invaded vs. reference marshes, we lost information on shifts in diversity patterns at the species/class level. We compared invertebrate class data within each meta-analysis study and found higher overall abundances of invertebrate prey in reference marshes for seven of the twelve prey groups (Table 1-3). In contrast, only two prey groups had higher overall abundances in $P$. australis, while the remaining three groups were neutral. The diet of the dominant salt marsh resident, Fundulus heteroclitus, consists primarily of nematoda, ostracoda, tanaidacea, insecta, amphipoda, copepoda, gastropoda, and polychaeta (reviewed in James-Pirri et al. 2001), the majority of 
which are likely to be found in higher abundances in Spartina spp. than P. australis marshes (Table 1-3). Therefore, fish are more likely to find larger quantities of preferred prey items in reference marshes, which could affect the condition and quantity of nekton and higher trophic levels (birds).

\section{Animal Condition and Quantity}

Nekton assemblages largely drove our analysis of animal condition and quantity due to the disproportionate number of studies conducted on nekton rather than invertebrate or bird taxa (125 total data points on nekton, 25 for invertebrates, 11 for birds; Fig. 16). For nekton, the marsh surface is an essential habitat needed for foraging, refuge, and reproduction (Weisberg and Lotrich 1982; Able and Hagan 2000, 2003), with densities on the marsh surface increasing with flood duration (Minello et al. 2012). Marsh surface changes associated with introduced $P$. australis (i.e., high above/belowground biomass and increased sedimentation rates resulting in marsh surface accretion) can significantly reduce flooding by $52 \%$ as well as decrease the depth and duration of water coverage, in turn restricting nekton access to the marsh surface (Osgood et al. 2003). In late invasion stages, accretion can raise the marsh surface above the elevation of the highest high tides eventually transforming the marsh into terrestrial habitat (Weis and Butler 2009). Our analysis of effect sizes revealed negative impacts of the invasive plant on the quantity, condition, and diversity of fauna using the marsh surface but not on those residing in tidal creeks, indicating that access to or the quality of the habitat has been compromised. In addition, our finding that fauna are negatively affected in open systems rather than tidally restricted 
marshes (Fig. 1-7) suggests that it is more likely that the invasive plant (rather than altered hydrologic flow) is the cause for negative effects seen in this particular analysis.

We detected disproportionate negative effects on larval and juvenile nekton. Phragmites australis fills in small marsh pools and water-filled depressions on the marsh surface (Windham and Lathrop 1999), thereby reducing nursery habitat and flushing larval fish into the main tidal creek where they may be exposed to predators (Able and Hagan 2003; Raichel et al. 2003). Reductions in flooding frequency and depth have been correlated with decreases in catch per unit effort for juvenile fish as the invasion progresses, from 51.6 fish in reference marshes to 2.4 in the late invasion stage (Hunter et al. 2006). Not only are juvenile fish more abundant in reference marshes (1,440 vs. 29), but the abundance of available invertebrate prey is higher (Raichel et al. 2003), suggesting that introduced $P$. australis may affect both the survival and recruitment of juvenile fish (Able and Hagan 2003; Osgood et al. 2003). Furthermore, the proportion of gravid $F$. heteroclitus residing in $P$. australis marshes is significantly reduced relative to non-invaded marshes (K.L. Dibble, unpublished data). This suggests that the combined long-term effects of reduced spawning, reduced recruitment, and reduced larval/juvenile survival in tidal creeks on fish populations may yet be realized.

Nekton often move onto the marsh surface on flood tide with empty guts and leave on ebb tide with full guts (Kneib 1986; Fell et al. 1998). Reduced access to prey 
inhabiting the marsh surface can decrease weight gain and the growth rate of resident fish in comparison to those only having access to the creek bed (Weisberg and Lotrich 1982), which can result in trade-offs to energy storage, growth, and reproduction that ultimately influences survival (Post and Parkinson 2001; Jorgensen et al. 2006). We detected differences in the overall condition of fauna inhabiting invaded marshes, which supports our marsh surface results. Reduced access can decrease foraging and expose fish to predators in the tidal creek system, in turn increasing energy expenditure, decreasing growth rate, and suppressing reproduction (Weisberg and Lotrich 1982; Fraser and Gilliam 1992; Brown et al. 2005).

Our sample size for the bird taxa was low due to lack of published studies that specifically compared $P$. australis to Spartina spp. However, even with a small sample size, our results generally indicate negative impacts on the quantity and diversity of birds utilizing the marsh surface in $P$. australis. This is likely due to the relationship between bird species richness and presence and extent of open marsh pools used for foraging (Benoit and Askins 1999; Trocki and Patton 2006), which are reduced in $P$. australis and less accessible due to the tall stature of the invasive plant (Windham and Lathrop 1999; Able et al. 2003). In addition, free-living herbivores and plant hoppers are reduced in $P$. australis in favor of concealed detritivores (Gratton and Denno 2005), which could affect the distribution and abundance of birds that consume insect prey. Further, marsh specialists adapted to nesting in short-statured graminoids, such as the seaside sparrow, saltmarsh sharp-tailed sparrow, and willet, can lose nesting habitat post-invasion (Benoit and Askins 1999). However, P. australis does not 
eliminate habitat for all bird species. Marsh generalists such as the red-winged blackbird are abundant in P. australis and the glossy ibis has been reported to nest in P. australis in comparison to upland habitats (Parsons 2003; Kiviat 2006; Barrett and Mcbrien 2007; Wells et al. 2008).

\section{Geographic Expansion and Future Effects on Biota}

We detected overall negative effects of the invasive plant on animal communities within the mid-Atlantic, but not in the New England region (Fig. 1-4), which is possibly related to plant cover and invasion stage. Kneib (2003) studied nekton production (via bioenergetic linkages) in relation to the availability of marsh/creek edge and found significant decreases at sites with little creek edge (as would be the case in highly invaded systems), with the highest production at sites where fish reside within 200 meters of marsh fringe. Although regional estimates of introduced $P$. australis cover and existing marsh fringe are not available for the mid-Atlantic and New England, several lines of evidence suggest that sites studied in the mid-Atlantic were in the later invasion stages, which as discussed earlier, correlates with increased faunal impact. The exact origin and timing of the plants introduction has not been definitively established; however, Burk (1877) documented a 2-3 year old stand of morphologically distinct $P$. australis growing on ballast in Philadelphia, PA while molecular analysis of an herbarium accession (J.C. Martindale, US-908070) revealed that a stand growing in Camden, NJ in 1877 was of non-native origin (Saltonstall 2002; Meadows and Saltonstall 2007). The authors in our meta-analysis intensely studied highly invaded sites located in Delaware Bay (Fig. 1-1, Table 1-1), a water 
body that culminates upstream at the cities of Philadelphia and neighboring Camden, NJ. Therefore, the negative effects seen in our meta-analysis for the mid-Atlantic region could possibly be due to invasion longevity, since stands of introduced $P$. australis date back to at least the 1870's in that watershed.

Reduced salinity $(<18 \mathrm{ppt})$ is one factor that contributes to the spread of $P$. australis (Chambers et al. 1999). A greater proportion of authors from the mid-Atlantic reported salinities at study sites in the oligohaline to mesohaline zone (0.3-16.0 ppt), while those in New England largely fell in the higher mesohaline to polyhaline zone. The expansion rate of introduced $P$. australis in oligohaline marshes is twofold (2.73-

$\left.2.92 \% \mathrm{yr}^{-1}\right)$ than that in higher salinity marshes (1.07-1.10\% $\mathrm{yr}^{-1}$; Warren et al. 2001), so conditions in the mid-Atlantic may have contributed to P. australis expansion. Although the plant is highly plastic and can grow in polyhaline conditions (Vasquez et al. 2005), higher salinity at sites in New England could have maintained fringing salt marsh edge and hence nekton support (Kneib 2003), potentially explaining the nonsignificance seen in the northern region.

\section{Restoration Potential}

Salt marsh restoration dating back to at least the 1980's has shown rapid recovery of faunal communities on a decadal time scale (Roman et al. 1984; Warren et al. 2002). Across all categorical variables, this analysis has shown that restoration efforts have successfully converted physically and biologically altered habitat to that seen in uninvaded reference marshes. Restoration success (as measured by the quantity, 
diversity, and condition of fauna) has been realized regardless of the type of restoration used (tidal hydrology or manual/physical/chemical removal). In some cases, restored habitats even conferred slightly better conditions for invertebrates and nekton on the marsh surface as indicated by positive, although non-significant, effect sizes not seen in any of the invaded comparisons (Figs. 1-3 through 1-7).

Our results agree with recent global meta-analyses on the effectiveness of restoration actions to increase biodiversity and ecosystem function in degraded systems (Rey Benayas et al. 2009; Moreno-Mateos et al. 2012). Using a range of ecosystem types, Rey Benayas et al. (2009) found that biodiversity (as measured by species abundance, growth, biomass, richness, and diversity) increased by $44 \%$ post-restoration. Although the restored systems were not equivalent to their reference state, log response ratios for the restored sites reached $86 \%$ of those in reference areas. Moreno-Mateos et al. (2012) specifically evaluated global wetland restoration success for fauna within salt marshes, depressional/lacustrine wetlands, peatlands, floodplains, and mangroves. They found that wetland vertebrate and macroinvertebrate communities were restored within 5-10 years and that, overall, wetlands that are hydrologically connected to others recovered more rapidly due to exchange of animals with reference marshes. Hydrologic restoration has been shown to increase the abundance of shorebirds by $1400 \%$ in the first year of restoration and by $1000 \%$ in the second year (Raposa 2008), as well as increase the abundance and diversity of marsh specialists, shorebirds, waterfowl, and long-legged wading birds over time (Brawley et al. 1998; Rochlin et al. 2012). Our collective results show that impacts in altered wetlands are reversible 
and ecological function can be restored quickly for fauna using our current set of restoration tools. These findings should be of great interest to restoration practitioners, particularly those that are currently making habitat management decisions regarding the control of this plant invader.

\section{Concluding Remarks}

Our results indicate that introduced $P$. australis has negatively affected faunal communities in the North American invaded range and that those effects vary geographically, taxonomically, by life history stage, and by marsh habitat type. However, adverse effects can be reversed relatively quickly for sites that maintain a hydrologic connection to reference marshes. Phragmites australis continues to spread into the south and Gulf coasts, with impacts to salt marshes and wetlands likely to be similar to those invaded in northern regions. The south Atlantic and Gulf of Mexico both have large expanses of brackish and salt marsh potentially available for colonization (362,000 and 1,000,000 ha, respectively; Fields et al. 1991 in Chambers et al. 1999). The south Atlantic is on the leading edge of the invasion, with the plant colonizing approximately $14.6 \%$ of the Maryland shoreline but only $2.0 \%$ of the Virginia shoreline in the Chesapeake Bay (Chambers et al. 2008). The Gulf coast represents a confluence or 'hot spot' of five distinct $P$. australis lineages co-existing, expanding, and (in some cases) hybridizing, with the potential creation of an even more aggressive strain of $P$. australis that could affect fauna as it colonizes new habitats and expands westward (Meyerson et al. 2010, in press; Lambertini et al. 2012). This is an ongoing invasion and therefore our results from the mid and northern 
regions of the Atlantic may have salience elsewhere. Although $P$. australis may ultimately provide benefits to fauna via marsh accretion that keeps pace with sea level rise, current reductions in habitat value as evidenced by this study combined with the loss of plant biodiversity warrant action to manage the invasion before fauna and trophic support functions are diminished.

\section{Acknowledgements}

We thank Carla Lambertini for her review of this manuscript. Many thanks to Peter August for drafting the map of research sites for studies used in our analysis. We also thank the following agencies and organizations for their support: Environmental Protection Agency Science To Achieve Results Graduate Fellowship Program (FP91710001-0), National Oceanic and Atmospheric Administration National Estuarine Research Reserve Graduate Fellowship Program (NA09NOS4200041), National Science Foundation Integrative Graduate Education and Research Traineeship Grant to the Coastal Institute at the University of Rhode Island (0504103), Philanthropic Educational Organization (Lellis-Dib3158688), Northeast Aquatic Plant Management

Society, Rhode Island Natural History Survey and The Nature Conservancy of Rhode Island (Lellis-Dibble 05-30-09), University of Rhode Island Agricultural Experiment Station (RI00H-332, 311000-6044), University of Rhode Island Coastal Fellows Program, and the U.S. and Czech Fulbright Commissions. 


\section{References}

Able KW, Hagan SM (2000) Effects of common reed (Phragmites australis) invasion on marsh surface macrofauna: response of fishes and decapod crustaceans. Estuaries 23(5): 633-646.

Able KW, Hagan SM (2003) Impact of common reed, Phragmites australis, on Essential Fish Habitat: influence on reproduction, embryological development, and larval abundance of mummichog (Fundulus heteroclitus). Estuaries 26(1): 40-50.

Able KW, Hagan SM, Brown SA (2003) Mechanisms of marsh habitat alteration due to Phragmites: response of young-of-the-year mummichog (Fundulus heteroclitus) to treatment for Phragmites removal. Estuaries 26(2B): 484-494.

Adams DC, Gurevitch J, Rosenberg MS (1997) Resampling tests for meta-analysis of ecological data. Ecology 78(5): 1277-1283.

Allen EA, Fell PE, Peck MA, Gieg JA, Gutiike CR, Newkirk MD (1994) Gut contents of common mummichogs, Fundulus heteroclitus L., in a restored impounded marsh and in natural reference marshes. Estuaries 17(2): 462-471.

Angradi TR, Hagan SM, Able KW (2001) Vegetation type and the intertidal macroinvertebrate fauna of a brackish marsh: Phragmites vs. Spartina. Wetlands 21(1): 75-92.

Arnqvist G, Wooster D (1995) Meta-analysis: synthesizing research findings in ecology and evolution. Trends Ecol Evol 10(6): 236-240.

Barrett KR, Mcbrien MA (2007) Chemical and biological assessment of an urban, estuarine marsh in northeastern New Jersey, USA. Environ Monit Assess 124: 63-88.

Benoit LK, Askins RA (1999) Impact of the spread of Phragmites on the distribution of birds in Connecticut tidal marshes. Wetlands 19(1): 194-208.

Brawley AH, Warren RS, Askins RA (1998) Bird use of restoration and reference marshes within the Barn Island Wildlife Management Area, Stonington, Connecticut, USA. Environ Manage 22(4): 625-633.

Brittain RA, Schimmelmann A, Parkhurst DF, Craft CB (2012) Habitat use by coastal birds inferred from stable carbon and nitrogen isotopes. Estuaries Coast 35: 633-645. 
Brown C, Gardner C, Braithwaite VA (2005) Differential stress responses in fish from areas of high- and low-predation pressure. J Comp Physiol B: Biochemistry and Molecular Biology 175: 305-312.

Buchsbaum RN, Catena J, Hutchins E, James-Pirri MJ (2006) Changes in salt marsh vegetation, Phragmites australis, and nekton in response to increased tidal flushing in a New England salt marsh. Wetlands 26(2): 544-557.

Burdick DM, Dionne M, Boumans RM, Short FT (1997) Ecological responses to tidal restorations of two northern New England salt marshes. Wetl Ecol Manag 4(2): 129-144.

Burk I (1877) List of plants recently collected on ship's ballast in the neighborhood of Philadelphia. P Acad Nat Sci Phila 29: 105-109.

Bushaw-Newton KL, Kreeger DA, Doaty S, Velinsky DJ (2008) Utilization of Spartina- and Phragmites-derived dissolved organic matter by bacteria and ribbed mussels (Geukensia demissa) from Delaware Bay salt marshes. Estuaries Coast 31: 694-703.

Canty A, Ripley B (2011) boot: Bootstrap R (S-Plus) Functions. R package version 1.3-3.

Chambers RM, Meyerson LA, Saltonstall K (1999) Expansion of Phragmites australis into tidal wetlands of North America. Aquat Bot 64: 261-273.

Chambers RM, Havens KJ, Killeen S, Berman M (2008) Common reed Phragmites australis occurrence and adjacent land use along estuarine shoreline in Chesapeake Bay. Wetlands 28(4): 1097-1103.

Currin CA, Wainright SC, Able KW, Weinstein MP, Fuller CM (2003) Determination of food web support and trophic position of the mummichog, Fundulus heteroclitus, in New Jersey smooth cordgrass (Spartina alterniflora), common reed (Phragmites australis), and restored salt marshes. Estuaries 26: 495-510.

Davidson AM, Jennions M, Nicotra AB (2011) Do invasive species show higher phenotypic plasticity than native species and, if so, is it adaptive? A metaanalysis. Ecol Lett 14: 419-431.

Davison AC, Hinkley DV (1997) Bootstrap Methods and Their Applications. Cambridge Univ. Press, Cambridge.

Deegan LA, Garritt RH (1997) Evidence for spatial variability in estuarine food webs. Mar Ecol Prog Ser 147: 31-47. 
Dionne M, Short FT, Burdick DM (1999) Fish utilization of restored, created, and reference salt-marsh habitat in the Gulf of Maine. Am Fish S S 22: 384-404.

Eberhardt AL, Burdick DM, Dionne M (2011) The effects of road culverts on nekton in New England salt marshes: implications for tidal restoration. Restor Ecol 19(6): 776-785.

Efron B (1987) Better bootstrap confidence intervals (with discussion). J Am Stat Assoc 82: 171-200.

Farnsworth EJ, Meyerson LA (2003) Comparative ecophysiology of four wetland plant species along a continuum of invasiveness. Wetlands 23(4): 750-762.

Fell PE, Weissbach SP, Jones DA, Fallon MA, Zeppieri JA, Faison EK, Lennon KA, Newberry KJ, Reddington LK (1998) Does invasion of oligohaline tidal marshes by reed grass, Phragmites australis (Cav.) Trin. ex Steud., affect the availability of prey resources for the mummichog, Fundulus heteroclitus L.? J Exp Mar Biol Ecol 222: 59-77.

Fraser DF, Gilliam JF (1992) Nonlethal impacts of predator invasion: facultative suppression of growth and reproduction. Ecology 73(3): 959-970.

Fry B (2006) Stable Isotope Ecology. Springer, USA.

Gaertner M, Den Breeyen A, Hui C, Richardson DM (2009) Impacts of alien plant invasions on species richness in Mediterranean-type ecosystems: a metaanalysis. Prog Phys Geog 33(3): 319-338.

Gratton C, Denno RF (2005) Restoration of arthropod assemblages in a Spartina salt marsh following removal of the invasive plant Phragmites australis. Restor Ecol 13(2): 358-372.

Gratton C, Denno RF (2006) Arthropod food web restoration following removal of an invasive wetland plant. Ecol Appl 16: 622-631.

Grothues TM, Able KW (2003a) Discerning vegetation and environmental correlates with subtidal marsh fish assemblage dynamics during Phragmites eradication efforts: interannual Trend Measures. Estuaries 26(2B): 574-586.

Grothues TM, Able KW (2003b) Response of juvenile fish assemblages in tidal salt marsh creeks treated for Phragmites removal. Estuaries 26(2B): 563-573.

Gurevitch J, Hedges LV (1999) Statistical issues in ecological meta-analyses. Ecology 80(4): 1142-1149. 
Hagan SM, Brown SA, Able KW (2007) Production of mummichog (Fundulus heteroclitus): response in marshes treated for common reed (Phragmites australis) removal. Wetlands 27(1): 54-67.

Hauber DP, Saltonstall K, White DA, Hood CS (2011) Genetic variation in the common reed, Phragmites australis, in the Mississippi River Delta marshes: evidence for multiple introductions. Estuaries Coast 34: 851-862.

Hedges LV, Olkin I (1985) Statistical methods for meta-analysis. Academic Press, Florida.

Hedges LV, Gurevitch J, Curtis P (1999) The meta-analysis of response ratios in experimental ecology. Ecology 80: 1150-1156.

Hendricks LG, Mossop HE, Kicklighter CE (2011) Palatability and chemical defense of Phragmites australis to the marsh periwinkle snail Littoraria irrorata. $\mathrm{J}$ Chem Ecol 37: 838-845.

Hesterberg T, Moore DS, Monaghan S, Clipson A, Epstein R (2005) Bootstrap Methods and Permutation Tests, 2nd edition. W. H. Freeman, New York.

Holt ER, Buchsbaum R (2000) Bird use of Phragmites australis in coastal marshes of northern Massachusetts. In: Pederson, J. (ed.) Marine bioinvasions: proceedings of a conference, January 24-27, 1999. MIT Sea Grant College Program, 00(2): pp. 232-240.

Hunter KL, Fox DA, Brown LM, Able KW (2006) Responses of resident marsh fishes to stages of Phragmites australis invasion in three mid Atlantic estuaries. Estuaries Coast 29(3): 487-498.

James-Pirri MJ, Raposa KB, Catena JG (2001) Diet composition of mummichogs, Fundulus heteroclitus, from restoring and unrestricted regions of a New England (U.S.A.) salt marsh. Estuar Coast Shelf S 53: 205-213.

Jivoff PR, Able KW (2003) Blue crab, Callinectes sapidus, response to the invasive common reed, Phragmites australis: abundance, size, sex ratio, and molting frequency. Estuaries 26(2B): 587-595.

Jorgensen C, Ernande B, Fiksen O, Dieckmann U (2006) The logic of skipped spawning in fish. Can J Fish Aquat Sci 63: 200-211.

Kalies EL, Chambers CL, Covington WW (2010) Wildlife responses to thinning and burning treatments in southwestern conifer forests: a meta-analysis. Forest Ecol Manag 259: 333-342. 
Kettenring KM, Reinhardt Adams C (2011) Lessons learned from invasive plant control experiments: a systematic review and meta-analysis. J Appl Ecol 48: 970-979.

Kimball ME, Able KW (2007) Nekton utilization of intertidal salt marsh creeks: tidal influences in natural Spartina, invasive Phragmites, and marshes treated for Phragmites removal. J Exp Mar Biol Ecol 346: 87-101.

Kimball ME, Able KW, Grothues TM (2010) Evaluation of long-term response of intertidal creek nekton to Phragmites australis (common reed) removal in oligohaline Delaware Bay salt marshes. Restor Ecol 18(5): 772-779.

Kiviat E (2006) Phragmites management sourcebook for the tidal Hudson River. Report to the Hudson River Foundation, New York, New York. Hudsonia Ltd., Annandale NY 12504 USA.

Kneib RT (1986) The role of Fundulus heteroclitus in salt marsh trophodynamics. Am Zool 26: 259-269.

Kneib RT (2003) Bioenergetic and landscape considerations for scaling expectations of nekton production from intertidal marshes. Mar Ecol Prog Ser 264: 279296.

Kulmatiski A, Beard KH, Meyerson LA, Gibson JC, Mock KE (2010) Nonnative Phragmites australis invasion into Utah wetlands. West N Am Naturalist 70(4): 541-552.

Lambertini C, Mendelssohn IA, Gustafsson MHG, Olesen B, Riis T, Sorrell BK, Brix H (2012) Tracing the origin of Gulf coast Phragmites (Poaceae): a story of long distance dispersal and hybridization. Am J Bot 99(3): 538-551.

Litvin SY, Weinstein MP (2003) Life history strategies of estuarine nekton: the role of marsh macrophytes, benthic microalgae, and phytoplankton in the trophic spectrum. Estuaries 26(2B): 552-562.

Litvin SY, Weinstein MP (2004) Multivariate analysis of stable-isotope ratios to infer movements and utilization of estuarine organic matter by juvenile weakfish (Cynoscion regalis). Can J Fish Aquat Sci 61: 1851-1861.

McClary M Jr. (2004) Spartina alterniflora and Phragmites australis as habitat for the ribbed mussel, Geukensia demissa (Dillwyn), in Saw Mill Creek of New Jersey's Hackensack Meadowlands. Urban Habitats 2(1): 83-90.

McKinley A, Johnston EL (2010) Impacts of contaminant sources on marine fish abundance and species richness: a review and meta-analysis of evidence from the field. Mar Ecol Prog Ser 420: 175-191. 
Meadows RE, Saltonstall K (2007) Distribution of native and introduced Phragmites australis in freshwater and oligohaline tidal marshes of the Delmarva Peninsula and southern New Jersey. J Torrey Bot Soc 134(1): 99-107.

Meyer DL, Johnson JM, Gill JW (2001) Comparison of nekton use of Phragmites australis and Spartina alterniflora marshes in the Chesapeake Bay, USA. Mar Ecol Prog Ser 209: 71-84.

Meyerson LA, Chambers RM, Vogt KA (1999) The effects of Phragmites removal on nutrient pools in a freshwater tidal marsh ecosystem. Biol Invasions 1: 129136.

Meyerson LA, Saltonstall K, Windham L, Kiviat E, Findlay S (2000) A comparison of Phragmites australis in freshwater and brackish marsh environments in North America. Wetl Ecol Manage 8: 89-103.

Meyerson LA, Viola D, Brown RN (2010) Hybridization of invasive Phragmites australis with a native subspecies in North America. Biol Invasions 12: $103-$ 111.

Meyerson LA, Lambertini C, McCormick MK, Whigham D (in press) Jambalaya on the Bayou? A review of hybridization in common reed in North America. Am J Bot.

Minello TJ, Rozas LP, Baker R (2012) Geographic variability in salt marsh flooding patterns may affect nursery value for fishery species. Estuaries Coast. 35: 501514.

Molloy PP, Reynolds JD, Gage MJG, Mosqueira I, Cote IM (2008) Links between sex change and fish densities in marine protected areas. Biol Conserv 141: 187197.

Moreno-Mateos D, Power ME, Comin FA, Yockteng R (2012) Structural and functional loss in restored wetland ecosystems. PLoS Biol 10(1). doi:10.1371/journal.pbio.1001247.

Mozder TJ, Zieman JC (2010) Ecophysiological differences between genetic lineages facilitate the invasion of non-native Phragmites australis in North American Atlantic coast wetlands. J Ecol 98: 451-458.

Osgood DT, Yozzo DJ, Chambers RM, Jacobson D, Hoffman T, Wnek J (2003) Tidal hydrology and habitat utilization by resident nekton in Phragmites and nonPhragmites marshes. Estuaries 26(2B): 522-533. 
Parsons KC (2003) Reproductive success of wading birds using Phragmites marsh and upland nesting habitats. Estuaries 26(2B): 596-601.

Peterson BJ (1999) Stable isotopes as tracers of organic matter input and transfer in benthic food webs: a review. Acta Oecol 20(4): 479-487.

Posey MH, Alphin TD, Meyer DL, Johnson JM (2003) Benthic communities of common reed Phragmites australis and marsh cordgrass Spartina alterniflora marshes in Chesapeake Bay. Mar Ecol Prog Ser 261: 51-61.

Post JR, Parkinson EA (2001) Energy allocation strategy in young fish: allometry and survival. Ecology 82(4): 1040-1051.

Powell KI, Chase JM, Knight TM (2011) A synthesis of plant invasion effects on biodiversity across spatial scales. Am J Bot 98(3): 539-548.

Pyšek P, Jarosik V, Hulme PE, Pergl J, Hejda M, Schaffner U, Vilà M (2012) A global assessment of invasive plant impacts on resident species, communities and ecosystems: the interaction of impact measures, invading species' traits and environment. Glob Change Biol. doi: 10.1111/j.1365-2486.2011.02636.x.

R Development Core Team (2011) R: A language and environment for statistical computing. R Foundation for Statistical Computing, Vienna, Austria. ISBN 3900051-07-0, URL: http://www.R-project.org/.

Raichel DL, Able KW, Hartman JM (2003) The influence of Phragmites (common reed) on the distribution, abundance, and potential prey of a resident marsh fish in the Hackensack Meadowlands, New Jersey. Estuaries 26(2B): 511-521.

Raposa K (2002) Early responses of fishes and crustaceans to restoration of a tidally restricted New England salt marsh. Restor Ecol 10(4): 665-676.

Raposa K (2008) Early ecological responses to hydrologic restoration of a tidal pond and salt marsh complex in Narragansett Bay, Rhode Island. J Coast Res 55: 180-192.

Raposa KB, Roman CT (2001) Seasonal habitat-use patterns of nekton in a tiderestricted and unrestricted New England salt marsh. Wetlands 21(4): 451-461.

Raposa KB, Roman CT (2003) Using gradients in tidal restriction to evaluate nekton community responses to salt marsh restoration. Estuaries 26(1): 98-105.

Rey Benayas JM, Newton AC, Diaz A, Bullock JM (2009) Enhancement of biodiversity and ecosystem services by ecological restoration: a meta-analysis. Science 325: 1121-1124. 
Robertson TL, Weis JS (2005) A comparison of epifaunal communities associated with the stems of salt marsh grasses Phragmites australis and Spartina alterniflora. Wetlands 25(1): 1-7.

Robertson TL, Weis JS (2007). Interactions between the grass shrimp Palaemonetes pugio and the salt marsh grasses Phragmites australis and Spartina alterniflora. Biol Invasions 9: 25-30.

Rochlin I, James-Pirri MJ, Adamowicz SC, Dempsey ME, Iwanejko T, Ninivaggi DV (2012) The effects of integrated marsh management (IMM) on salt marsh vegetation, nekton, and birds. Estuaries Coast. 35: 727-742.

Roman CT, Niering WA, Warren RS (1984) Salt marsh vegetation change in response to tidal restriction. Environ Manage 8: 141-150.

Roman CT, Raposa KB, Adamowicz SC, James-Pirri MJ, Catena JG (2002) Quantifying vegetation and nekton response to tidal restoration of a New England salt marsh. Restor Ecol 10(3): 450-460.

Rooth JE, Court Stevenson J, Cornwell JC (2003) Increased sediment accretion rates following invasion by Phragmites australis: the role of litter. Estuaries 26(2B): 475-483.

Saltonstall K (2002) Cryptic invasion by a non-native genotype of the common reed, Phragmites australis, into North America. P Natl Acad Sci 99: 2445-2449.

Sorte CJB, Williams SL, Carlton JT (2010) Marine range shifts and species introductions: comparative spread rates and community impacts. Global Ecol Biogeogr 19: 303-316.

Stribling JM, Cornwell JC (1997) Identification of important primary producers in a Chesapeake Bay tidal creek system using stable isotopes of carbon and sulfur. Estuaries 20(1): 77-85.

Talley TS, Levin LA (2001) Modification of sediments and macrofauna by an invasive marsh plant. Biol Invasions 3: 51-68.

Trocki CL, Paton PWC (2006) Assessing habitat selection by foraging egrets in salt marshes at multiple spatial scales. Wetlands 26(2): 307-312.

Vasquez EA, Glenn EP, Brown JJ, Guntenspergen GR, Nelson SG (2005) Salt tolerance underlies the cryptic invasion of North American salt marshes by an introduced haplotype of the common reed Phragmites australis (Poaceae). Mar Ecol Prog Ser 298: 1-8. 
Vilà M, Espinar J, Hejda M, Hulme PE, Jarosik V, Maron JL, Pergl J, Schaffner U, Sun Y, Pyšek P (2011) Ecological impacts of invasive alien plants: a metaanalysis of their effects on species, communities, and ecosystems. Ecol Lett 14: 702-708.

Wainright SC, Weinstein MP, Able KW, Currin CA (2000) Relative importance of benthic microalgae, phytoplankton, and the detritus of smooth cordgrass Spartina alterniflora and the common reed Phragmites australis to brackishmarsh food webs. Mar Ecol Prog Ser 200: 77-91.

Warren RS, Fell PE, Grimsby JL, Buck EL, Rilling GC, Fertik RA (2001) Rates, patterns, and impacts of Phragmites australis expansion and effects of experimental control on vegetation, macroinvertebrates, and fish within tidelands of the Lower Connecticut River. Estuaries 24(1): 90-107.

Warren RS, Fell PE, Rozsa R, Brawley AH, Orsted AC, Olson ET, Swamy V, Niering WA (2002) Salt marsh restoration in Connecticut: 20 years of science and management. Restor Ecol 10(3): 497-513.

Weinstein MP, Litvin SY, Bosley KL, Fuller CM, Wainright SC (2000) The role of tidal marsh as an energy source for marine transient and resident finfishes: a stable isotope approach. T Am Fish Soc 129: 797-810.

Weinstein MP, Keough JR, Gutenspergen GR, Litvin SY (2003) Phragmites australis: a sheep in wolf's clothing? Estuaries 26(2B): 397.

Weinstein MP, Litvin SY, Guida VG (2009) Essential Fish Habitat and wetland restoration success: a tier III approach to the biochemical condition of common mummichog Fundulus heteroclitus in common reed Phragmites australis and smooth cordgrass Spartina alterniflora-dominated salt marshes. Estuaries Coast 32: 1011-1022.

Weis JS, Weis P (2000) Behavioral responses and interactions of estuarine animals with an invasive marsh plant: a laboratory analysis. Biol Invasions 2: 305-314.

Weis JS, Windham L, Santiago-Bass C, Weis P (2002) Growth, survival, and metal content of marsh invertebrates fed diets of detritus from Spartina alterniflora Loisel. and Phragmites australis Cav. Trin. ex Steud. from metal-contaminated and clean sites. Wetl Ecol Manag 10: 71-84.

Weis JS (2005) Diet and food web support of the white perch, Morone americana, in the Hackensack Meadowlands of New Jersey. Environ Biol Fishes 74: 109113.

Weis JS, Butler CA (2009) Salt marshes: a natural and unnatural history. Rutgers Univ. Press, New Jersey. 
Weisberg SB, Lotrich VA (1982) The importance of an infrequently flooded intertidal marsh surface as an energy source for the mummichog Fundulus heteroclitus: an experimental approach. Mar Biol 66: 307-310.

Wells AW, Nieder WC, Swift BL, O’Connor KA, Weiss CA (2008) Temporal changes in the breeding bird community at four Hudson River tidal marshes. J Coast Res 55: 221-235.

Whiteway SL, Biron PM, Zimmerman A, Venter O, Grant JWA (2010) Do in-stream restoration structures enhance salmonid abundance? A meta-analysis. Can J Fish Aquat Sci 67: 831-841.

Windham L, Lathrop RG Jr. (1999) Effects of Phragmites australis (common reed) invasion on aboveground biomass and soil properties in brackish tidal marsh of the Mullica River, New Jersey. Estuaries 22(4): 927-935.

Windham L, Meyerson LA (2003) Effects of common reed (Phragmites australis) expansions on nitrogen dynamics of tidal marshes in the Northeastern U.S. Estuaries 26(2B): 452-464.

Woolcott CA (2005) Nekton use of Spartina alterniflora and Phragmites australis in the Hackensack Meadowlands. MS Thesis, Rutgers.

Wozniak AS, Roman CT, Wainright SC, McKinney RA, James-Pirri MJ (2006) Monitoring food web changes in tide-restored salt marshes: a carbon stable isotope approach. Estuaries Coast 29(4): 568-578.

Yuhas CE, Hartman JM, Weis JS (2005) Benthic communities in Spartina alterniflora- and Phragmites australis- dominated salt marshes in the Hackensack Meadowlands, New Jersey. Urban Habitats 3(1): 158-191. 


\section{TABLES: CHAPTER 1}

Table 1-1. Legend for geographic map (Fig. 1-1).

\begin{tabular}{|c|c|c|}
\hline MID-ATLANTIC & $\begin{array}{l}\text { \# STUDIES IN } \\
\text { SYSTEM }\end{array}$ & $\begin{array}{l}\text { MAP } \\
\text { ID }\end{array}$ \\
\hline Monie Creek, MD & 2 & 1 \\
\hline Grasonville Creeks, MD & 3 & 2 \\
\hline Mill Creek, NJ & 1 & 3 \\
\hline Mad Horse Creek, NJ & 5 & 4 \\
\hline Alloway Creek, NJ & 13 & 5 \\
\hline Blackbird Creek, DE & 1 & 6 \\
\hline Browns Run, NJ & 1 & 7 \\
\hline Sea Breeze, NJ & 1 & 8 \\
\hline West Creek, NJ & 2 & 9 \\
\hline Dennis Creek, NJ & 2 & 10 \\
\hline Hog Islands, NJ & 5 & 11 \\
\hline Horseshoe Cove, NY & 2 & 12 \\
\hline Hackensack Meadowlands, NJ & 8 & 13 \\
\hline Piermont Marsh, NY & 2 & 14 \\
\hline Accabonac Harbor, NY & 4 & 16 \\
\hline Mid-Atlantic Sampling Effort & $\begin{array}{c}52 \\
\text { (26 in Delaware Bay) }\end{array}$ & \\
\hline NEW ENGLAND & $\begin{array}{l}\text { \# STUDIES IN } \\
\text { SYSTEM }\end{array}$ & $\begin{array}{c}\text { MAP } \\
\text { ID }\end{array}$ \\
\hline Charles Wheeler Marsh, CT & 1 & 15 \\
\hline Back and Lieutenant Rivers, CT & 3 & 17 \\
\hline Barn Island, CT & 4 & 18 \\
\hline Galilee, RI & 2 & 19 \\
\hline Sachuest Point, RI & 4 & 20 \\
\hline Potter Pond, Prudence Island, RI & 1 & 21 \\
\hline Herring River, MA & 1 & 22 \\
\hline Hatches Harbor, MA & 3 & 23 \\
\hline Argilla Marsh, MA & 1 & 24 \\
\hline Bass Beach, NH & 1 & 25 \\
\hline Parsons Creek, NH & 1 & 26 \\
\hline Awcomin Marsh, NH & 2 & 27 \\
\hline Great Bay Marshes, NH & 2 & 28 \\
\hline Little River, NH & 1 & 29 \\
\hline Browns River Marsh, NH & 1 & 30 \\
\hline Drakes Island, ME & 3 & 31 \\
\hline New England Sampling Effort & 31 & \\
\hline
\end{tabular}


Table 1-2. Response variables included in the invaded and restored meta-analyses.

\begin{tabular}{|l|l|}
\hline Response Variable & Categories \\
\hline Metric & Quantity \\
& Condition \\
& Diversity \\
\hline Geographic region & Mid-Atlantic \\
& New England \\
\hline Geographic region/metric & Mid-Atlantic: quantity \\
& Mid-Atlantic: condition \\
& Mid-Atlantic: diversity \\
& New England: quantity \\
& New England: condition \\
& New England: diversity \\
\hline Taxon & Nekton \\
& Bird \\
& Invertebrate \\
\hline Life history stage & Sub-adult \\
& Adult \\
\hline Habitat sampled & Tidal creek \\
& Marsh surface \\
\hline Tidal/hydrologic regime & Open \\
& Restricted \\
\hline Restoration type* & Hydrologic \\
& Conventional \\
\hline
\end{tabular}

* Restored meta-analysis only. 
Table 1-3. Number of studies in which the invertebrate prey exhibited higher raw abundances in Spartina spp. or $P$. australis habitat (e.g., 5 studies quantified a higher raw abundance of oligochaeta in Spartina spp., whereas 1 study quantified a higher abundance of the prey in introduced $P$. australis). See Online Resource 1, Table 1-A1 for Study Identifiers in Parentheses.

\begin{tabular}{|c|c|c|}
\hline Invertebrate Prey & Spartina spp. & P. australis \\
\hline Polychaeta & $3(26,28,43)$ & $3(5,27,36)$ \\
\hline Oligochaeta & $5 \quad(5,26,27,28,36)$ & $1(43)$ \\
\hline Nemertea & 1 (36) & $1(5)$ \\
\hline Nematoda & $2(28,43)$ & $1(5)$ \\
\hline Gastropoda & $3(5,27,36)$ & $3(12,26,28)$ \\
\hline Ostracoda & $2(5,28)$ & 0 \\
\hline Arachnida & $4(5,13,27,36)$ & $3(26,28,43)$ \\
\hline Tanaidacea & $2(5,27)$ & 0 \\
\hline Insecta & $5(5,13,27,28,36)$ & $2(26,43)$ \\
\hline Copepoda & 1 (28) & $2(5,43)$ \\
\hline Amphipoda & $5_{(12,13,26,27,43)}$ & $3(5,28,36)$ \\
\hline Isopoda & $2(5,27)$ & $5(12,26,28,36,4$ \\
\hline
\end{tabular}




\section{FIGURES: CHAPTER 1}

Figure 1-1. Geographic locations of authors' study sites (see Table 1-1 for location identifiers). Map: Dr. Peter August, University of Rhode Island, Environmental Data Center.

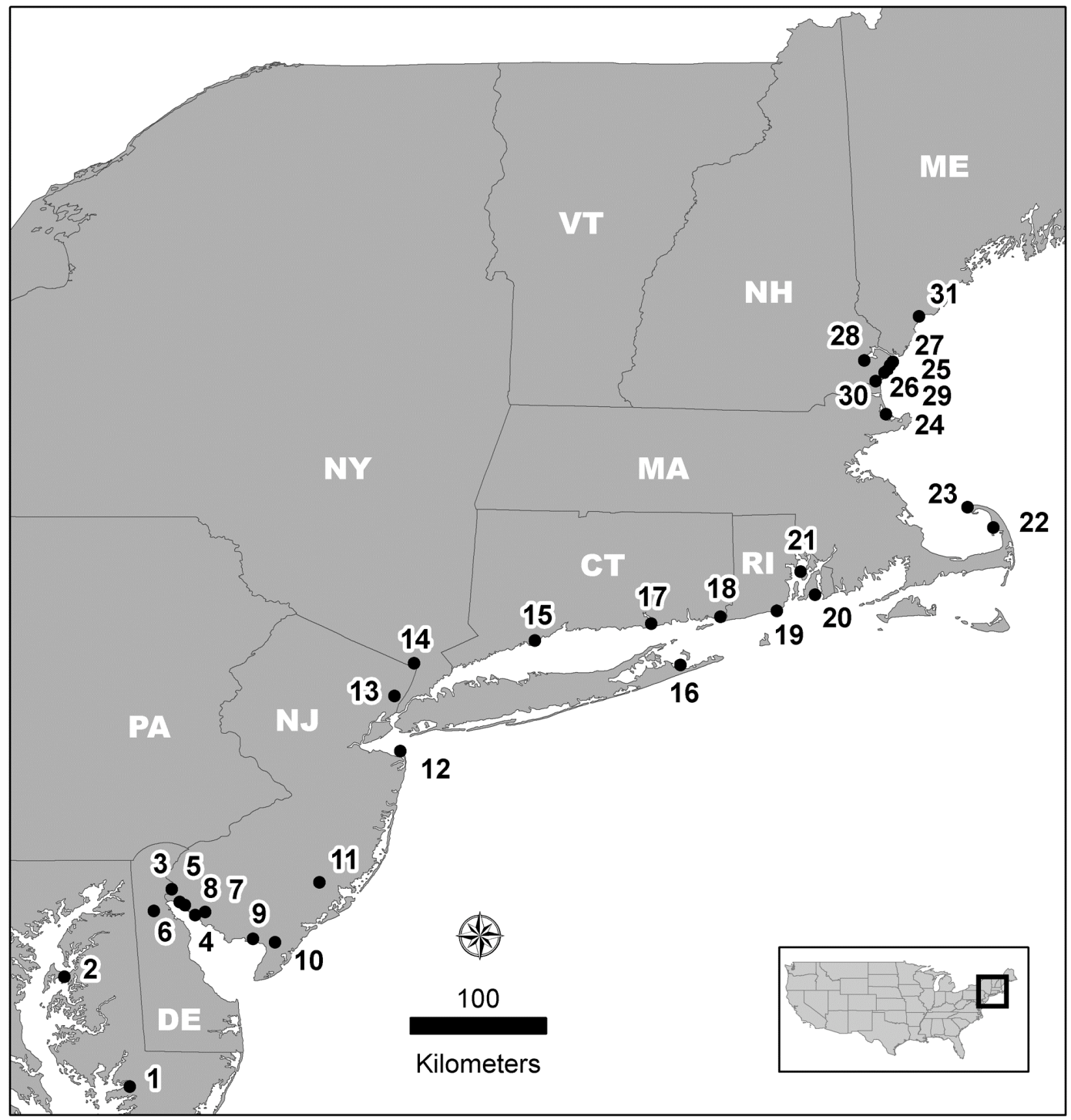


Figure 1-2. Number of studies in the literature concluding that introduced $P$. australis has negative, positive, or no effect (neutral) on habitat quality for salt marsh biota at invaded and restored sites. Number of studies are at the end of the bar graph segments. Note: some studies were counted more than once if the author presented mixed results (i.e., better habitat for one taxon or life history stage but not for another; Online Resource 1, Table 1-A1 study \#'s: 1, 21, 26, 32, 42).

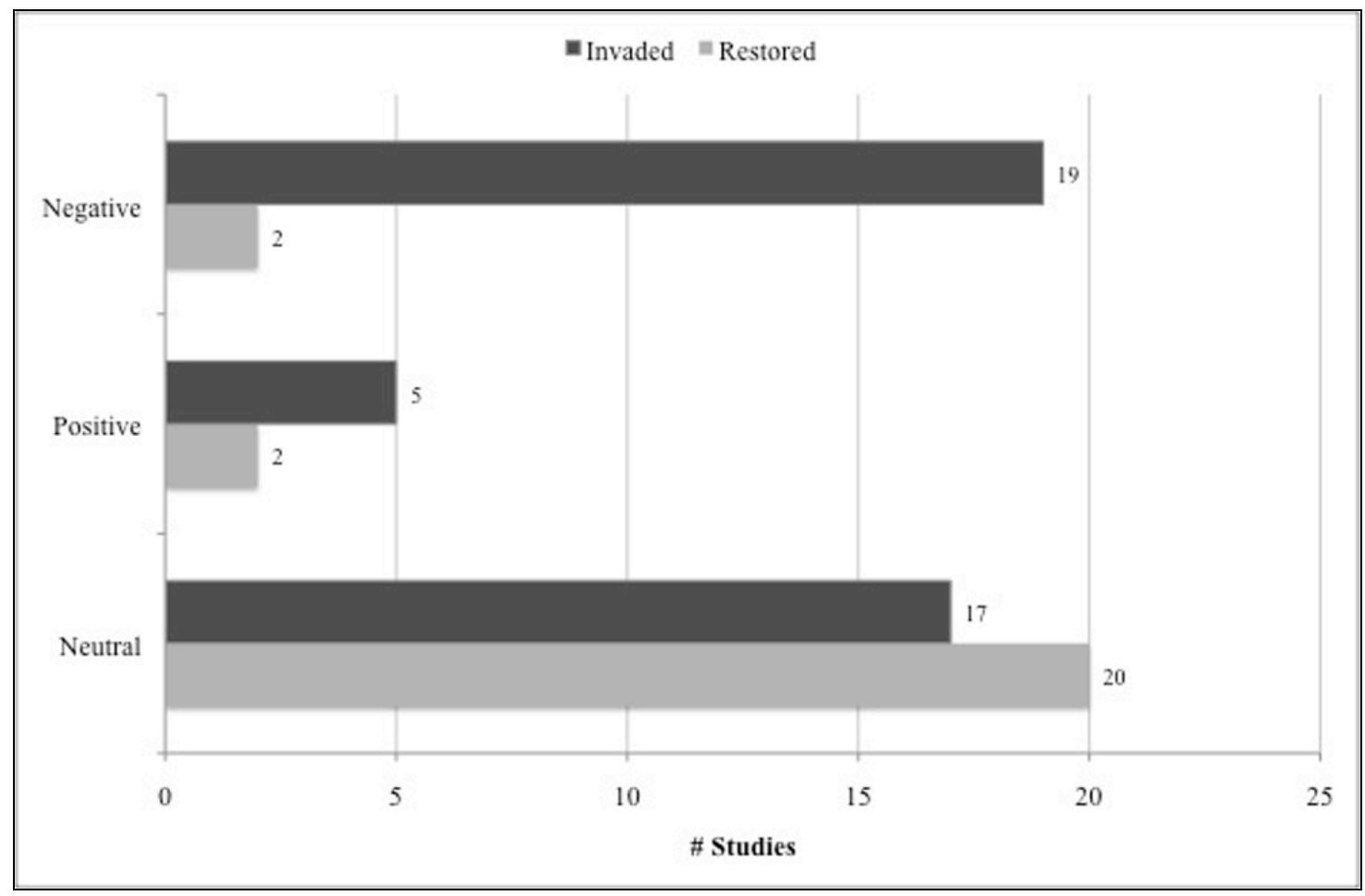


Figure 1-3. Results of meta-analysis by the 'metric' categorical variable. Mean effect sizes are black circles; negative effect sizes indicate fauna is negatively affected in introduced $P$. australis marshes in comparison to those residing in nearby reference Spartina spp. marshes. Zero is neutral, meaning no difference in faunal patterns. Horizontal lines are $95 \%$ bias-corrected bootstrap confidence intervals. Intervals overlapping zero are not significant. Sample sizes for each category are in parentheses.

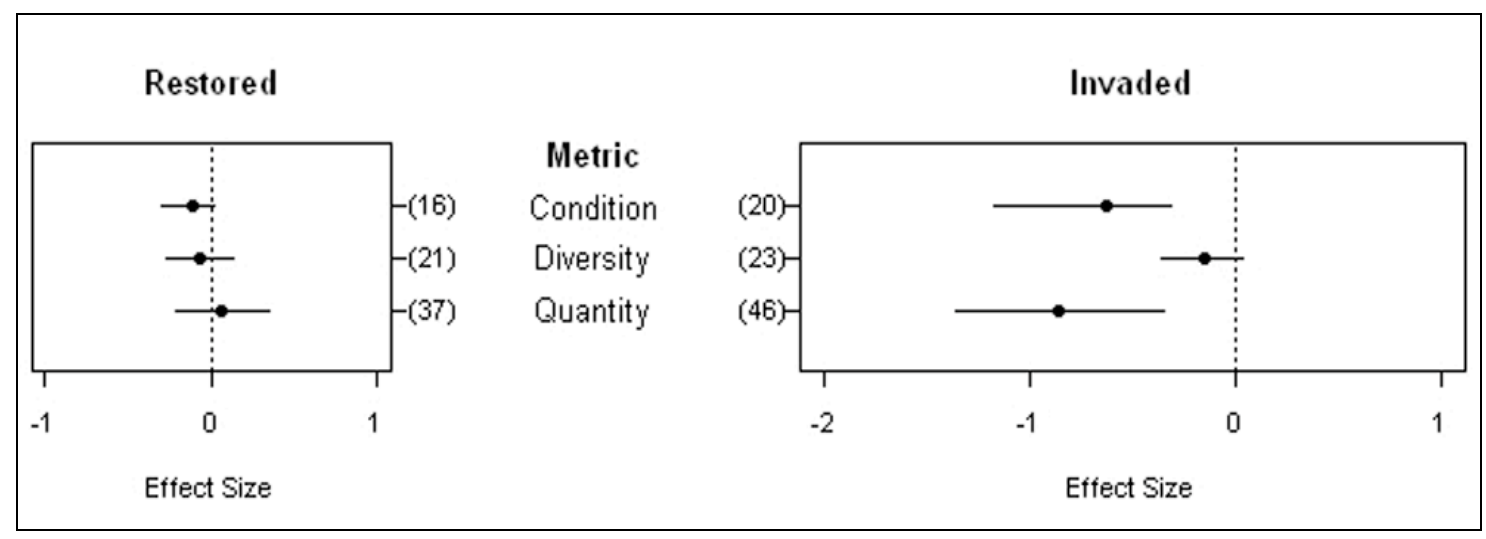


Figure 1-4. Regional differences in effect size on fauna residing in restored and invaded systems.

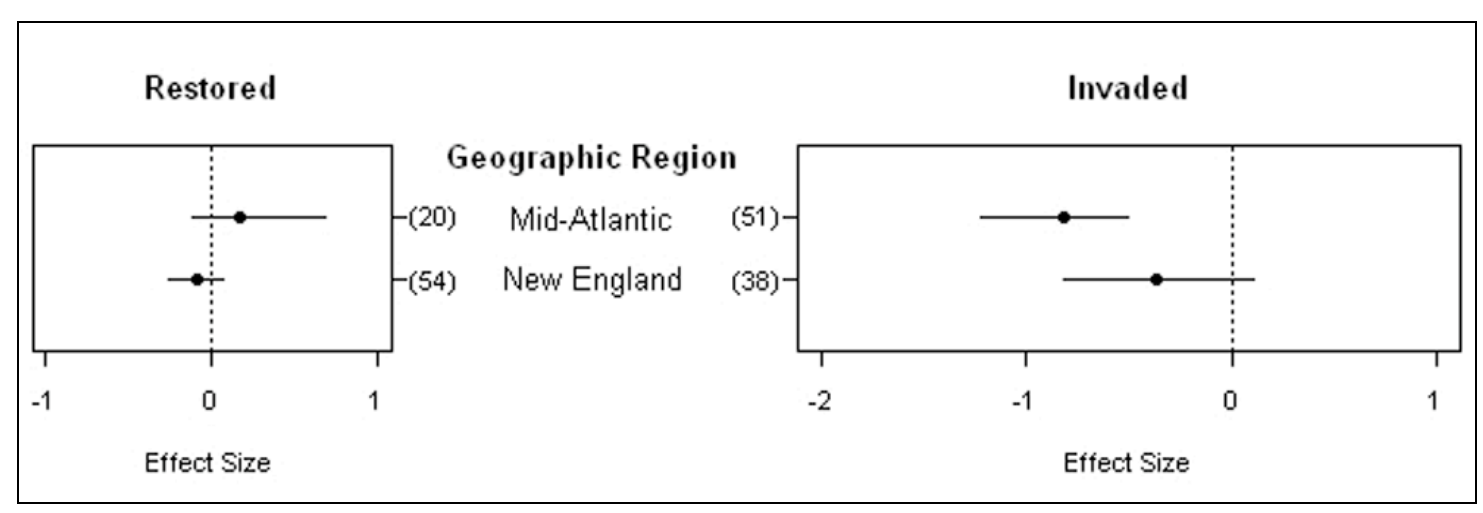


Figure 1-5. Regional differences by response metric in invaded and restored wetlands. Black circles without confidence intervals are mean effect sizes only and indicate we could not reliably calculate confidence limits due to small sample size. However, means are in plot to show relationship between all effect sizes for the restored and invasion studies.

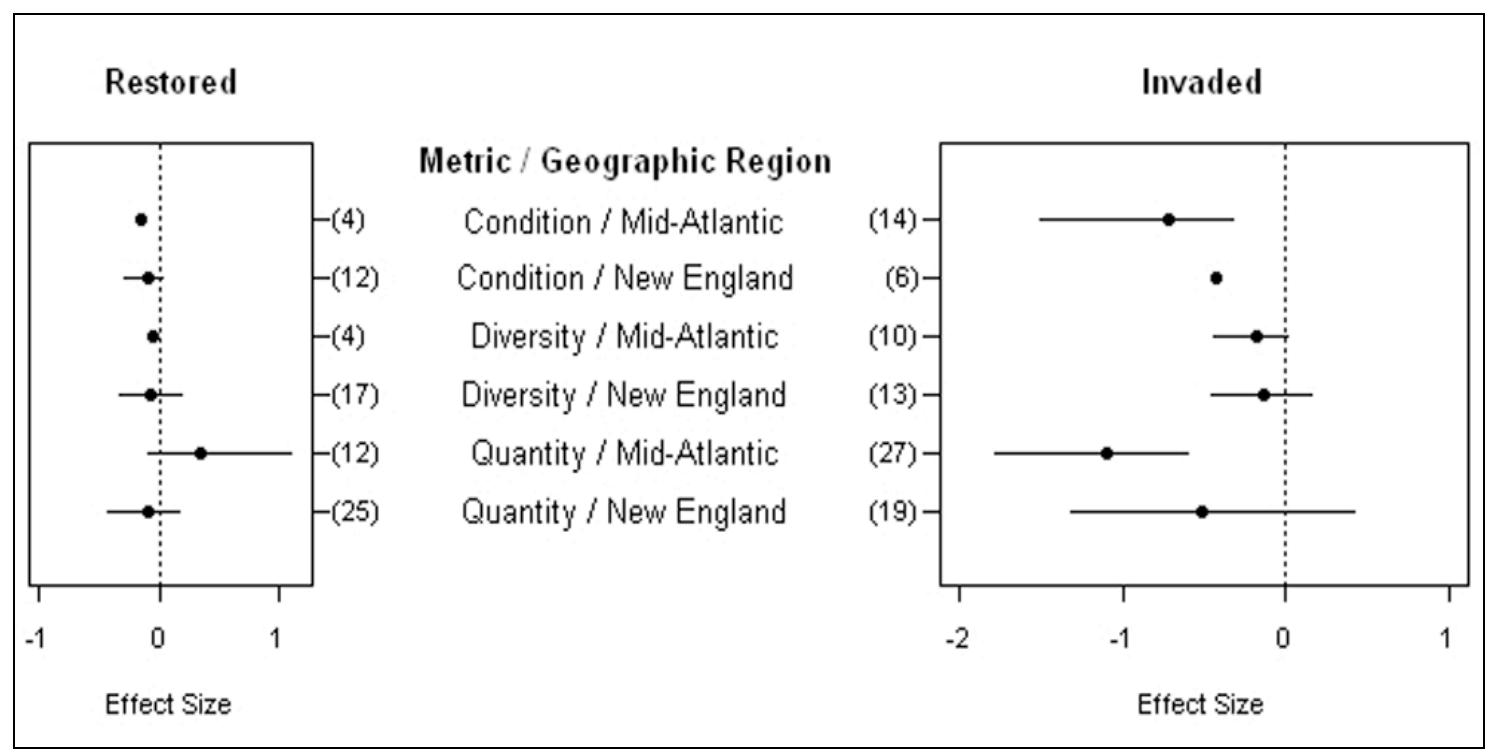


Figure 1-6. Meta-analysis results by taxon and life history stage. Black circles without confidence intervals are mean effect sizes only due to small sample size.

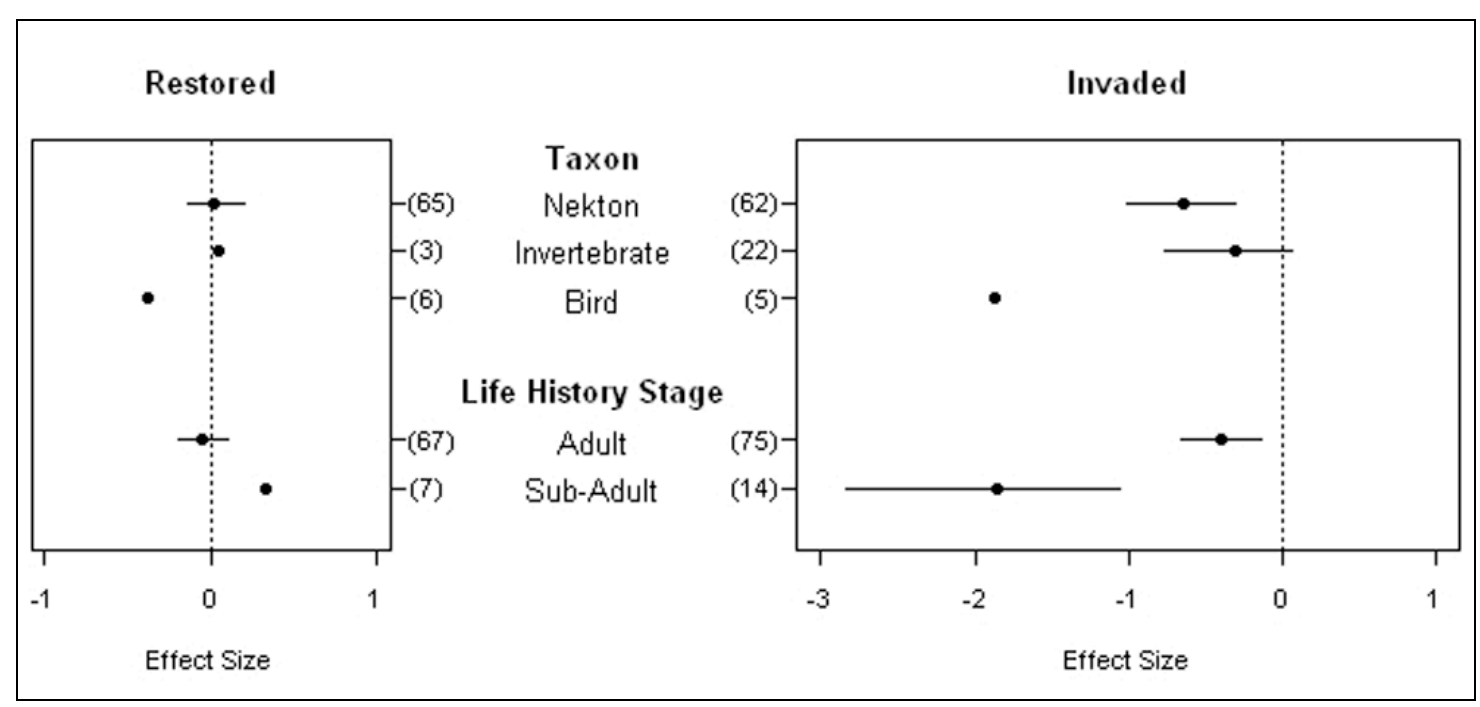


Figure 1-7. Meta-analysis results by marsh habitat and system type.

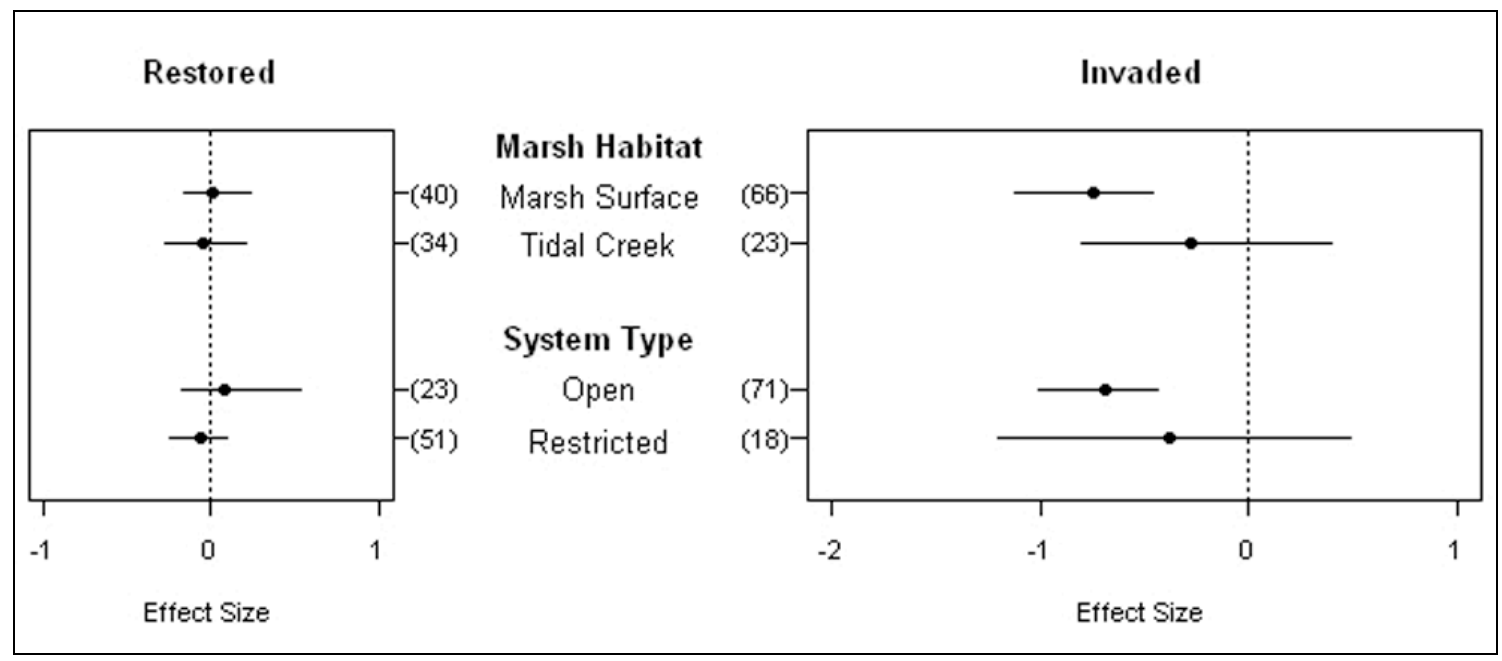


Figure 1-8. Differences in faunal patterns in hydrologically vs. conventionally restored sites.

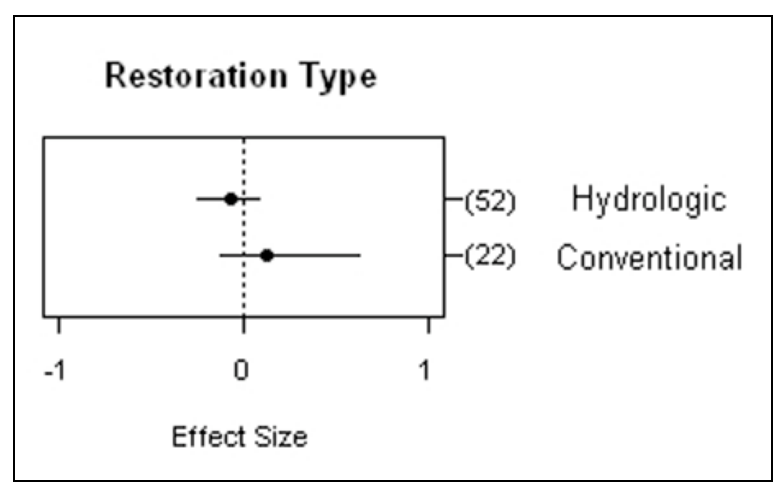




\section{APPENDIX: CHAPTER 1 (“Online Resource 1")}

Table 1-A1. Studies included in the meta-analysis.

\begin{tabular}{|c|c|c|c|c|c|c|c|c|c|c|}
\hline 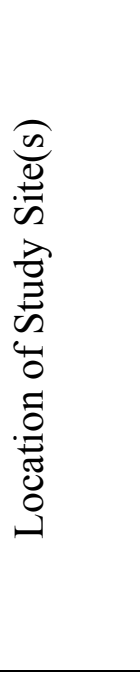 & 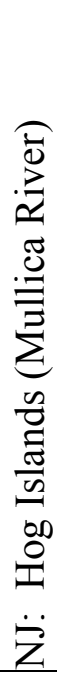 & 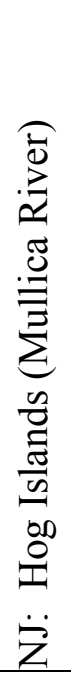 & 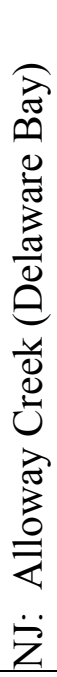 & 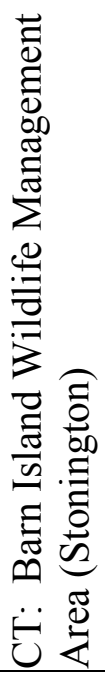 & 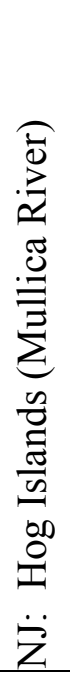 & 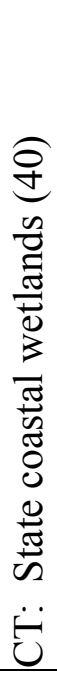 & 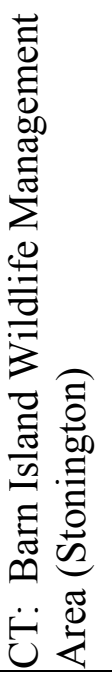 & 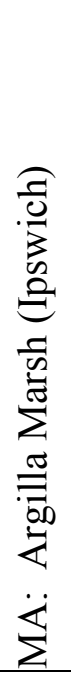 & 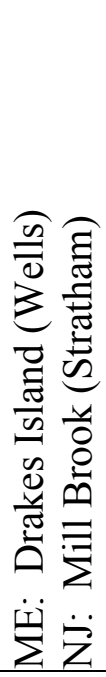 & 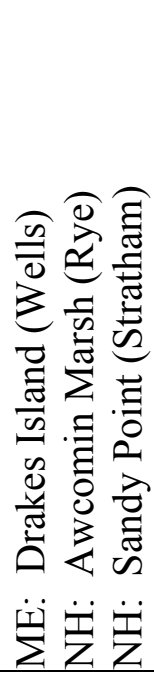 \\
\hline 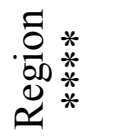 & $i^{2}$ & $\stackrel{\nabla}{\Sigma}$ & $\stackrel{D}{\Sigma}$ & 完 & $\sum^{\cdot D}$ & 崩 & $\frac{1}{Z}$ & 㞱 & 㞱 & 崩 \\
\hline 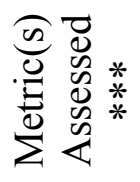 & 0 & $\begin{array}{l}0 \\
0\end{array}$ & 0 & U & $\begin{array}{l}0 \\
0\end{array}$ & $\begin{array}{l}0 \\
0\end{array}$ & $\begin{array}{l}0 \\
0\end{array}$ & 0 & $\begin{array}{l}0 \\
0 \\
0\end{array}$ & $\begin{array}{l}0 \\
0 \\
0\end{array}$ \\
\hline 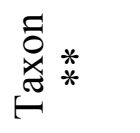 & Z & Z & Z & Z & - & $\boldsymbol{n}$ & 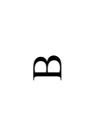 & Z & Z & Z \\
\hline 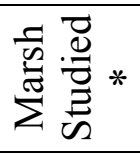 & - & $\neg$ & $\stackrel{\simeq}{\simeq}$ & $\simeq$ & 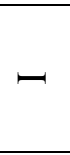 & 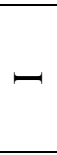 & $\simeq$ & $\stackrel{\sim}{\sim}$ & $\simeq$ & $\simeq$ \\
\hline 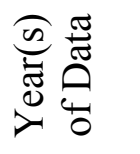 & $\sim$ & $N$ & 一 & 一 & - & $\sim$ & $N$ & 6 & $m$ & $\nabla$ \\
\hline 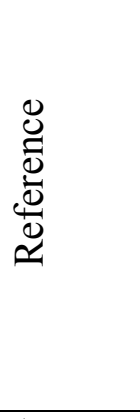 & 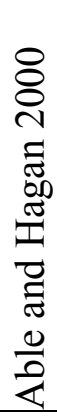 & 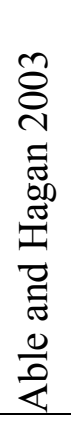 & 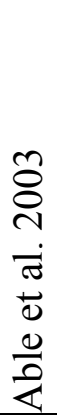 & 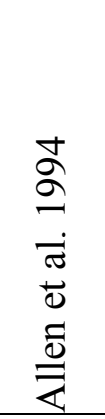 & 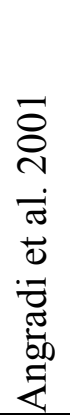 & 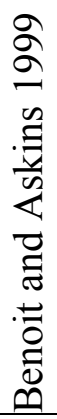 & 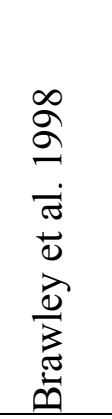 & 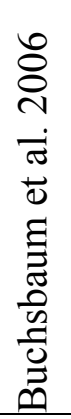 & 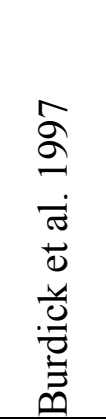 & 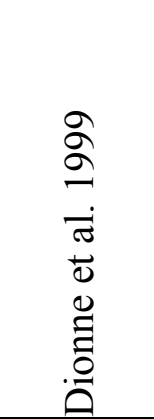 \\
\hline 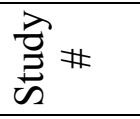 & 一 & $N$ & $m$ & $\nabla$ & $n$ & 6 & $r$ & $\infty$ & $a$ & 으 \\
\hline
\end{tabular}


Table 1-A1, cont.

\begin{tabular}{|c|c|c|c|c|c|c|c|c|}
\hline 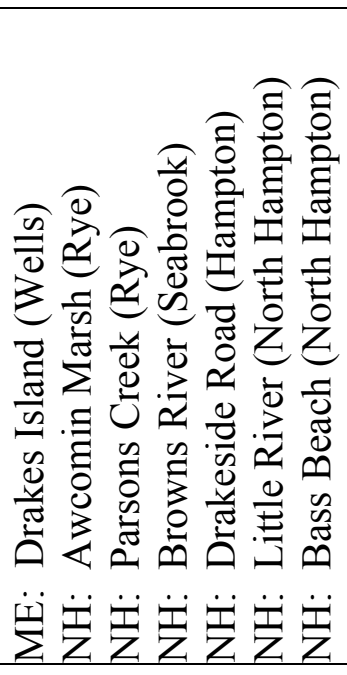 & 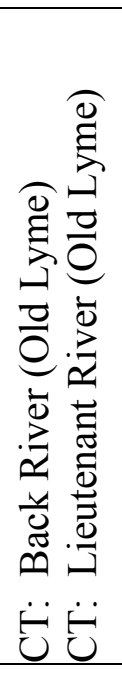 & 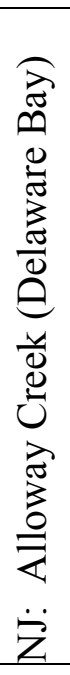 & 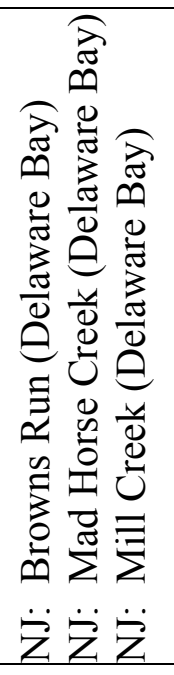 & 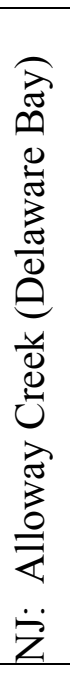 & 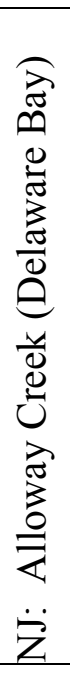 & 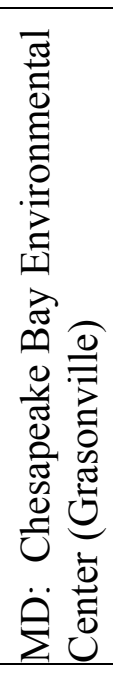 & 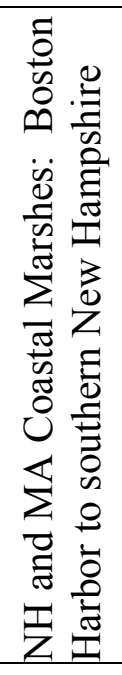 & 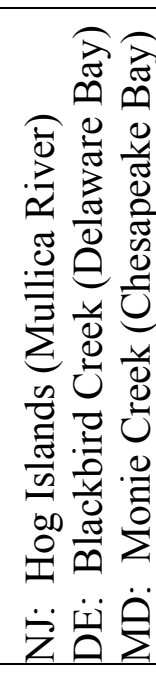 \\
\hline$\underline{\Sigma}$ & $\underline{\Sigma}$ & $\vec{\nabla}$ & $\stackrel{\nabla}{\Sigma}$ & $\vec{\nabla}$ & $\vec{\nabla}$ & $\stackrel{\nabla}{\Sigma}$ & 㞱 & $\stackrel{\nabla}{\Sigma}$ \\
\hline 0 & $\begin{array}{l}0 \\
0 \\
0\end{array}$ & $\begin{array}{l}0 \\
0\end{array}$ & $\begin{array}{l}0 \\
0\end{array}$ & 0 & $\begin{array}{l}0 \\
0\end{array}$ & U & O & $\begin{array}{l}0 \\
0\end{array}$ \\
\hline z & $z$ & - & Z & $z$ & $\mathrm{z}$ & - & 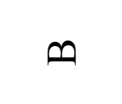 & z \\
\hline$\stackrel{r}{\sim}$ & $\stackrel{\simeq}{\sim}$ & $\approx$ & $\simeq$ & $\stackrel{\sim}{\simeq}$ & $\stackrel{\sim}{\sim}$ & - & - & - \\
\hline$N$ & - & - & in & $n$ & $\sim$ & - & $\sim$ & - \\
\hline 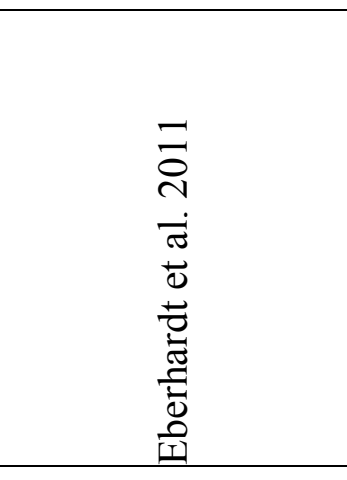 & $\begin{array}{l}\infty \\
2 \\
\stackrel{\sigma}{\sigma} \\
\pm \\
\overline{0} \\
\overline{0}\end{array}$ & 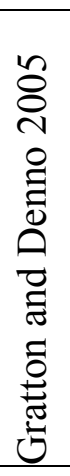 & 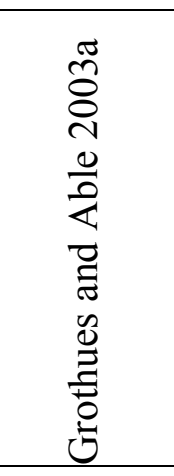 & 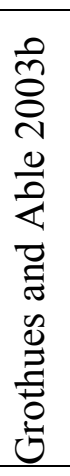 & 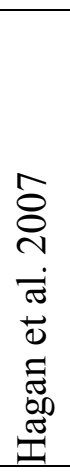 & 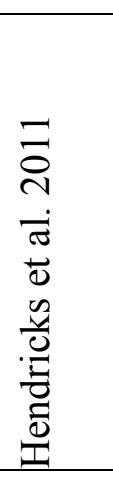 & 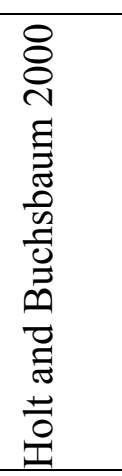 & 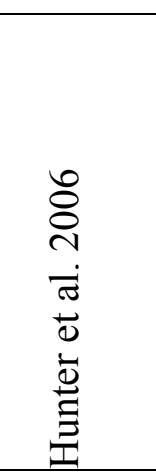 \\
\hline$=$ & $\simeq$ & 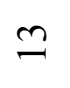 & $\Xi$ & $\cong$ & 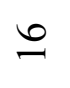 & 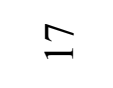 & $\stackrel{\infty}{-}$ & 2 \\
\hline
\end{tabular}


Table 1-A1, cont.

\begin{tabular}{|c|c|c|c|c|c|c|c|c|c|c|c|}
\hline 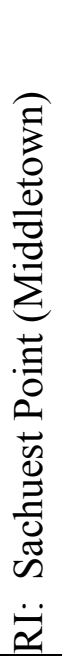 & 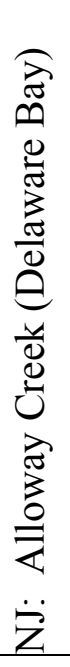 & 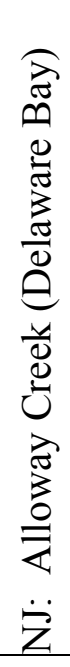 & 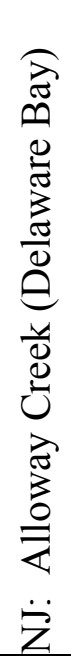 & 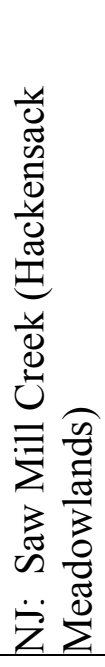 & 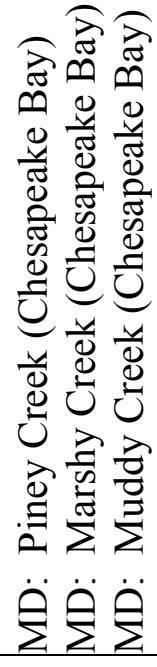 & 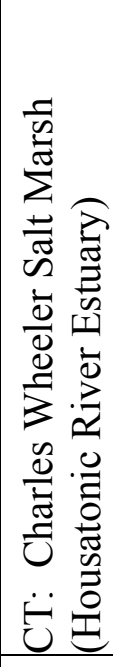 & 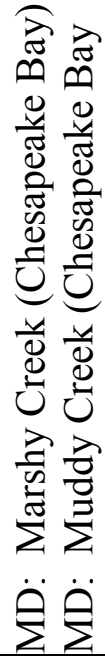 & 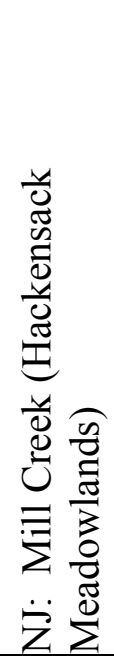 & 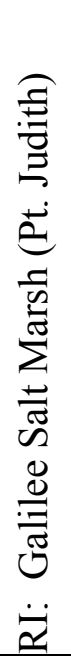 & 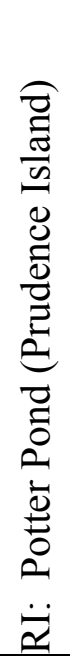 & 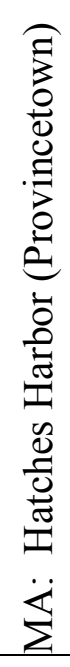 \\
\hline 㞱 & $\stackrel{\nabla}{\Sigma}$ & $\stackrel{\nabla}{\Sigma}$ & i & I & 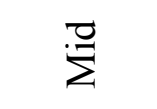 & 㞱 & $\stackrel{\nabla}{\Sigma}$ & $\vec{\nabla}$ & 㞱 & 乙 & 㞱 \\
\hline$U$ & 0 & 0 & 0 & $\sigma$ & $\begin{array}{l}0 \\
0 \\
0\end{array}$ & $\begin{array}{l}0 \\
\dot{a}\end{array}$ & 0 & $\begin{array}{l}0 \\
0\end{array}$ & $\stackrel{a}{a}$ & $\begin{array}{l}a \\
0\end{array}$ & $\begin{array}{l}0 \\
0\end{array}$ \\
\hline Z & $z$ & $z$ & Z & - & z & Z & - & Z & Z & $\begin{array}{l}z \\
n\end{array}$ & z \\
\hline$\simeq$ & $\stackrel{\simeq}{\simeq}$ & $\stackrel{x}{\sim}$ & $\stackrel{\sim}{\sim}$ & - & 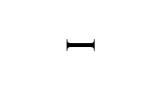 & - & - & - & $\stackrel{4}{\sim}$ & $\stackrel{\sim}{=}$ & - \\
\hline- & $n$ & - & $r$ & $\sim$ & - & - & - & $N$ & $m$ & $m$ & $N$ \\
\hline 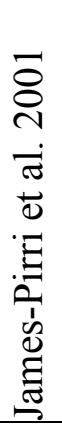 & 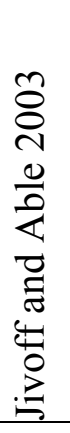 & 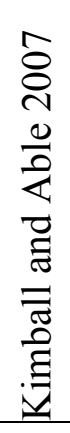 & 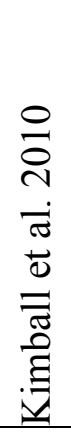 & 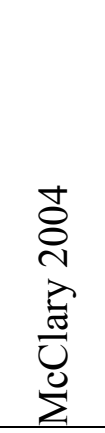 & 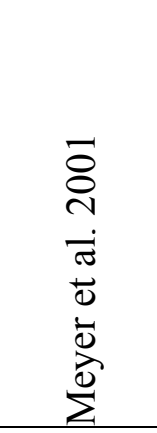 & 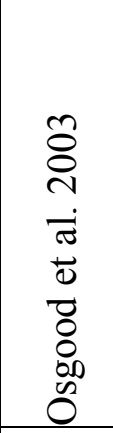 & 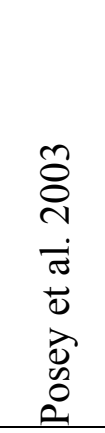 & 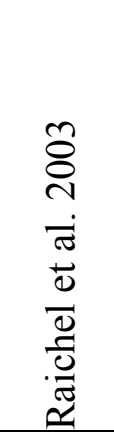 & 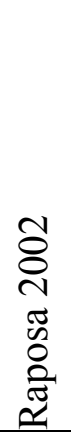 & 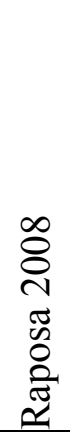 & 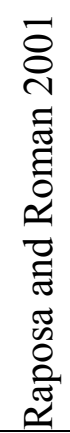 \\
\hline ¿ & $\vec{\sim}$ & $\approx$ & $\stackrel{\sim}{\sim}$ & $\stackrel{\Delta}{\sim}$ & $\approx$ & $\stackrel{\sim}{\sim}$ & $\hat{\sim}$ & $\stackrel{\infty}{\sim}$ & ৯े & లి & $\bar{n}$ \\
\hline
\end{tabular}


Table 1-A1, cont.

\begin{tabular}{|c|c|c|c|c|c|c|c|}
\hline 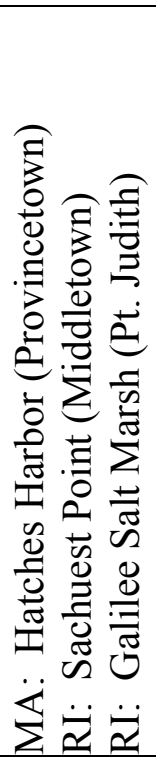 & 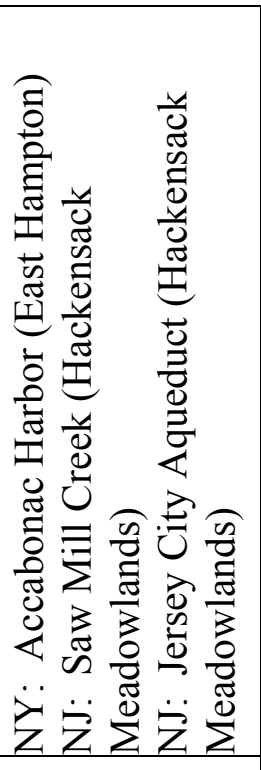 & 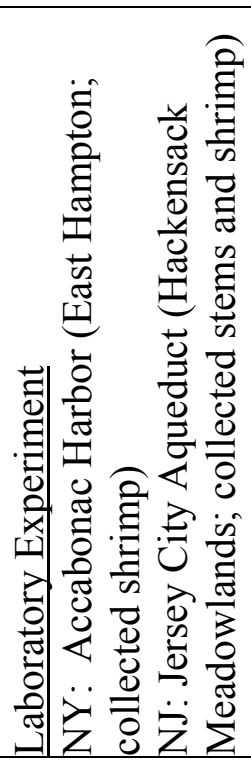 & 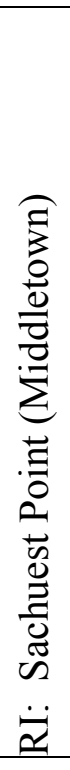 & 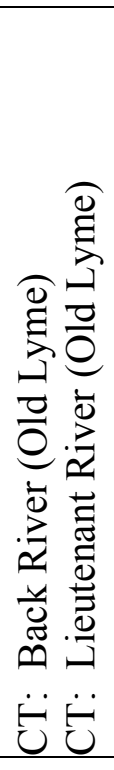 & 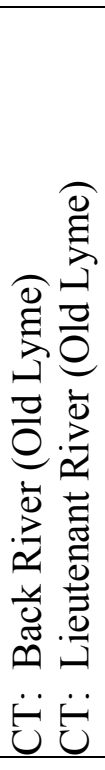 & 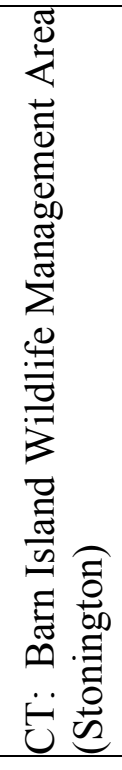 & 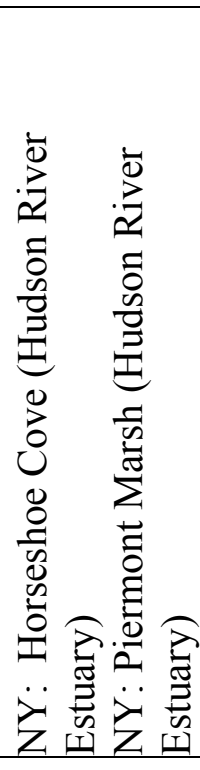 \\
\hline 㞱 & 疋 & . & 㞱 & $\underline{Z}$ & $\underline{\Sigma}$ & Z & 疋 \\
\hline$\stackrel{a}{a}$ & D & $u$ & $\begin{array}{l}0 \\
0\end{array}$ & $\begin{array}{l}0 \\
0 \\
0\end{array}$ & $\begin{array}{l}0 \\
0\end{array}$ & $\begin{array}{l}0 \\
0\end{array}$ & U \\
\hline Z & - & z & Z & - & z & $\begin{array}{l}z \\
n^{\prime}\end{array}$ & z \\
\hline$\simeq$ & 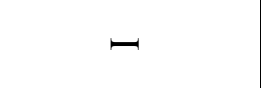 & - & $\stackrel{\simeq}{\sim}$ & $\neg$ & $\stackrel{\simeq}{\simeq}$ & $\simeq$ & - \\
\hline$m$ & $m$ & - & $m$ & - & - & $\sim$ & - \\
\hline 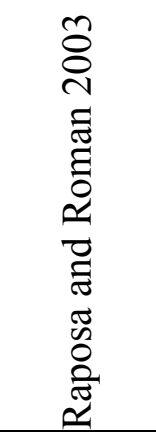 & 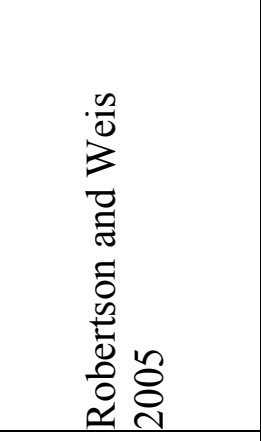 & 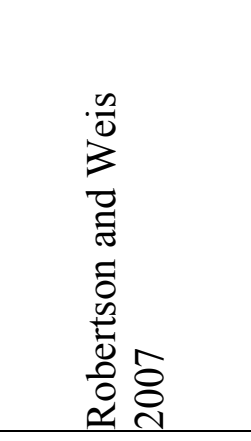 & 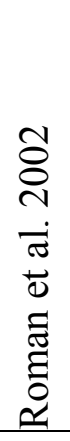 & 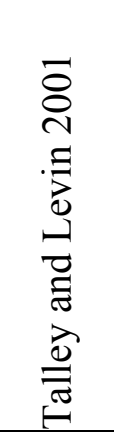 & 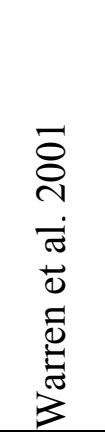 & 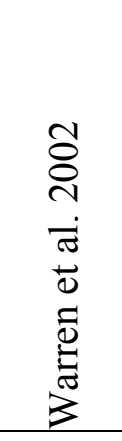 & 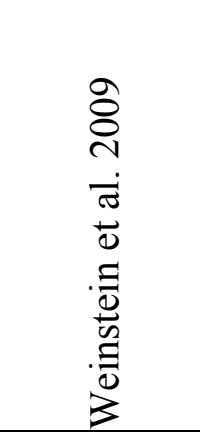 \\
\hline m & $m$ & m & $\stackrel{n}{n}$ & r & nn & $\stackrel{\infty}{n}$ & ले \\
\hline
\end{tabular}


Table 1-A1, cont.

\begin{tabular}{|c|c|c|c|c|}
\hline 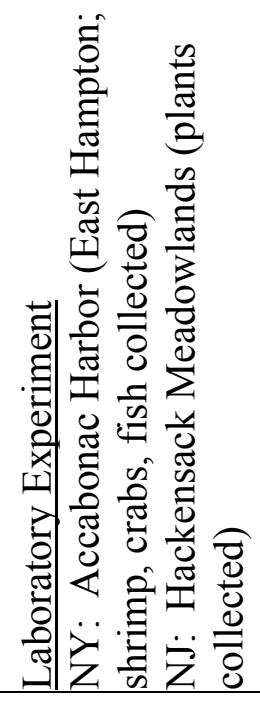 & 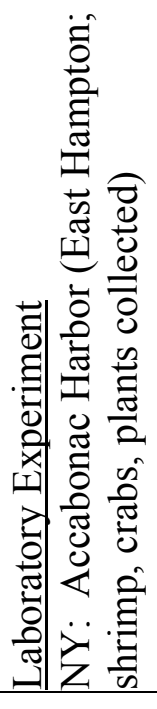 & 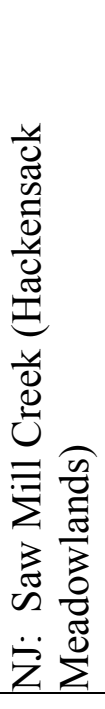 & 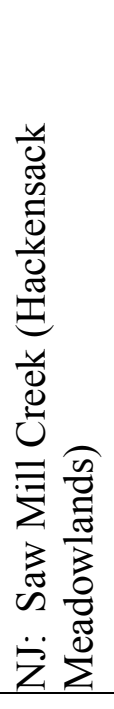 & 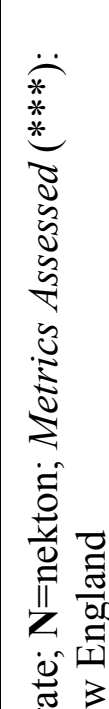 \\
\hline$\stackrel{\nabla}{\Sigma}$ & $\stackrel{\nabla}{\Sigma}$ & Z & $\stackrel{\nabla}{\Sigma}$ & $\begin{array}{l}0 \\
0 \\
0 \\
0\end{array}$ \\
\hline 0 & $U$ & $\begin{array}{l}0 \\
0^{n} \\
u^{n}\end{array}$ & $\begin{array}{l}a \\
0\end{array}$ & 密要 \\
\hline z & z & z & - & * \\
\hline- & $\stackrel{\simeq}{\simeq}$ & - & - & $\ddot{\nabla}$ \\
\hline- & - & - & - & $\simeq$ \\
\hline 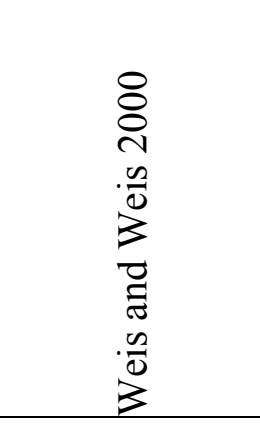 & 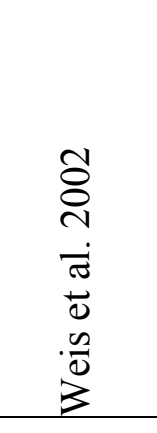 & 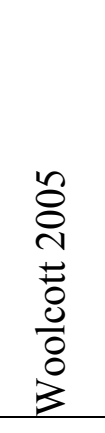 & 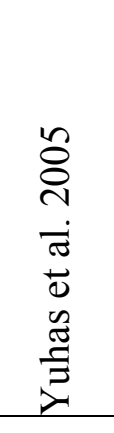 & 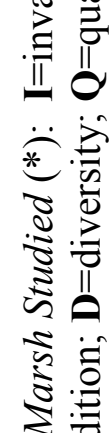 \\
\hline \& & $\vec{\gamma}$ & f & $\mathscr{f}$ & 空 \\
\hline
\end{tabular}


Table 1-A2. Stable isotope studies reviewed, with their primary conclusion.

\begin{tabular}{|c|c|c|c|c|}
\hline 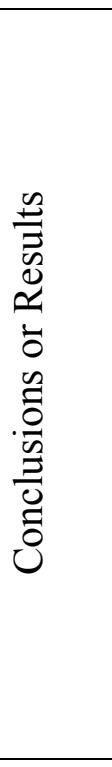 & 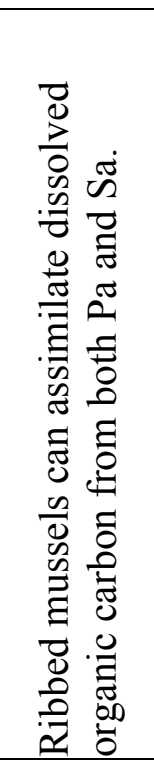 & 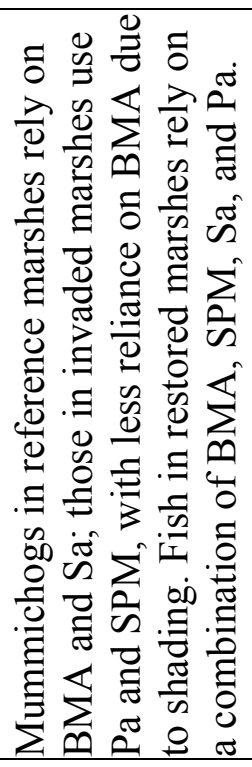 & 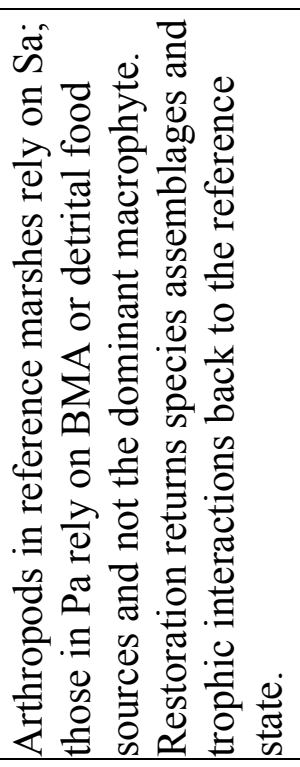 & 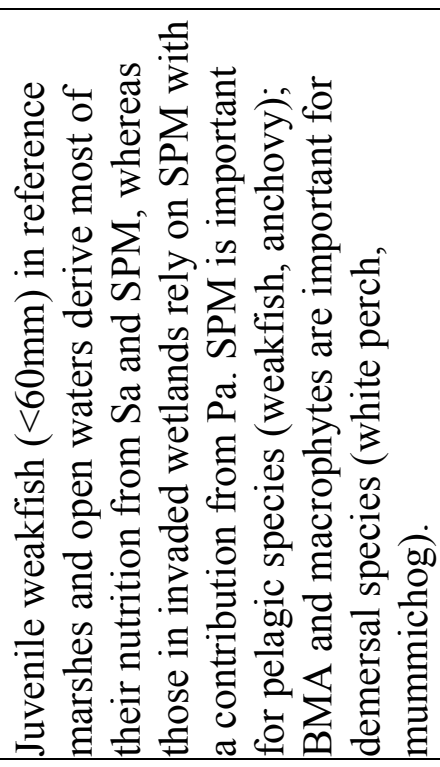 \\
\hline 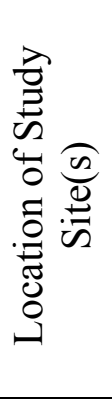 & 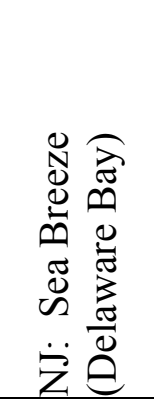 & 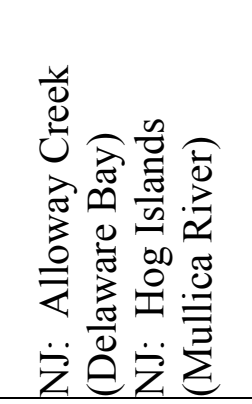 & 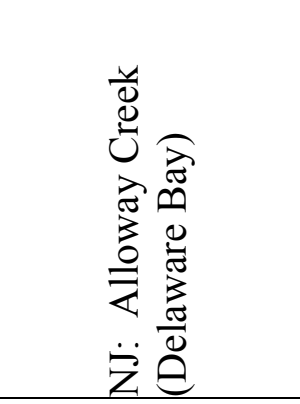 & 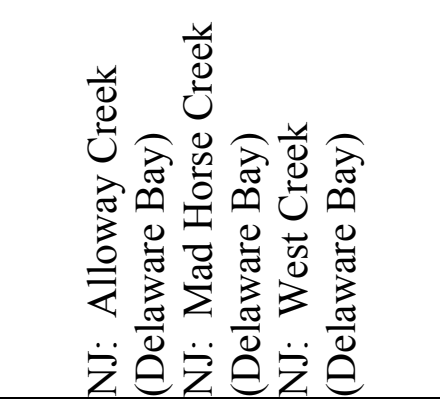 \\
\hline 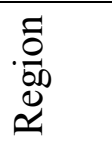 & $\underset{\Sigma}{\stackrel{D}{z}}$ & $\stackrel{\nabla}{\Sigma}$ & $\stackrel{D}{i}$ & $\stackrel{D}{i}$ \\
\hline 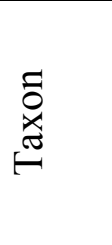 & 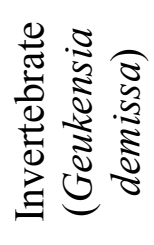 & 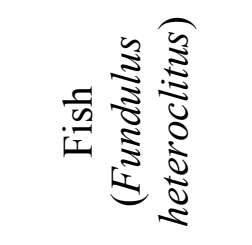 & 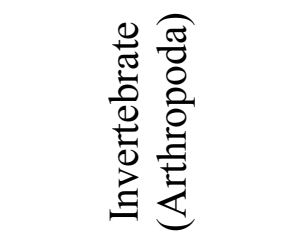 & 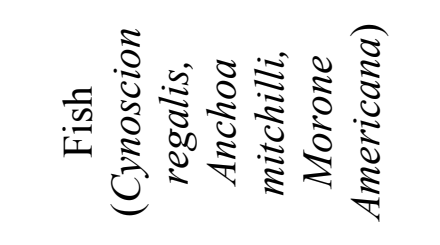 \\
\hline 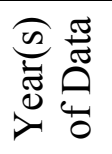 & $-\stackrel{\text { ఠ }}{8}$ & 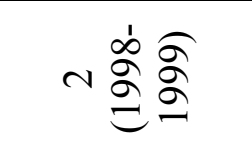 & $-\stackrel{\text { }}{\stackrel{8}{8}}$ & 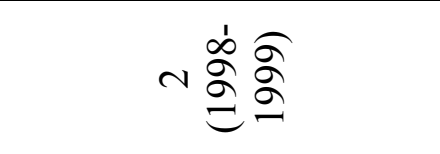 \\
\hline 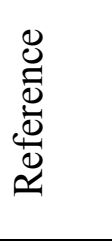 & 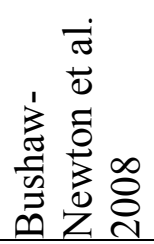 & 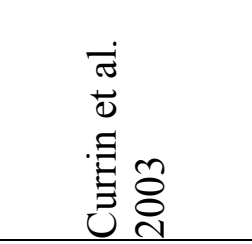 & 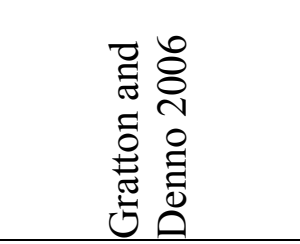 & 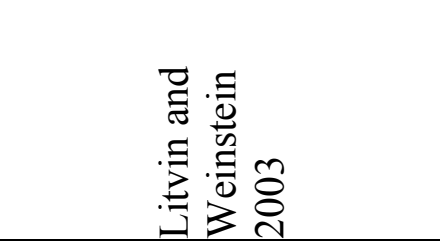 \\
\hline 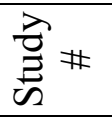 & $\forall$ & $\checkmark$ & f & $\checkmark$ \\
\hline
\end{tabular}


Table 1-A2, cont.

\begin{tabular}{|c|c|c|c|}
\hline 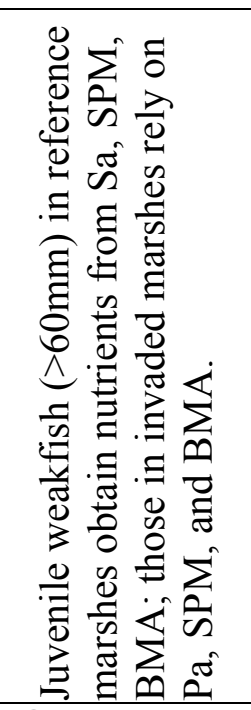 & 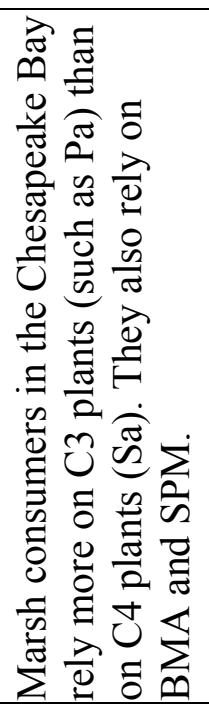 & 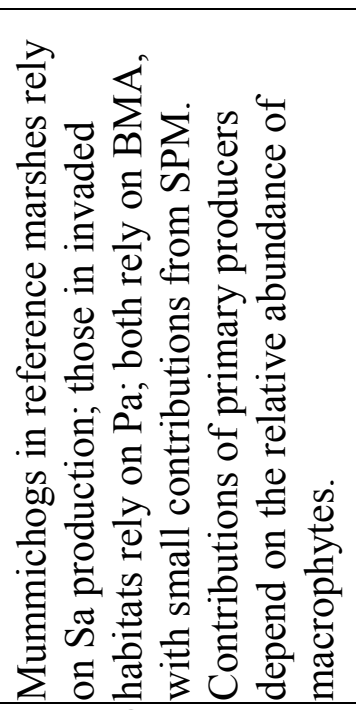 & 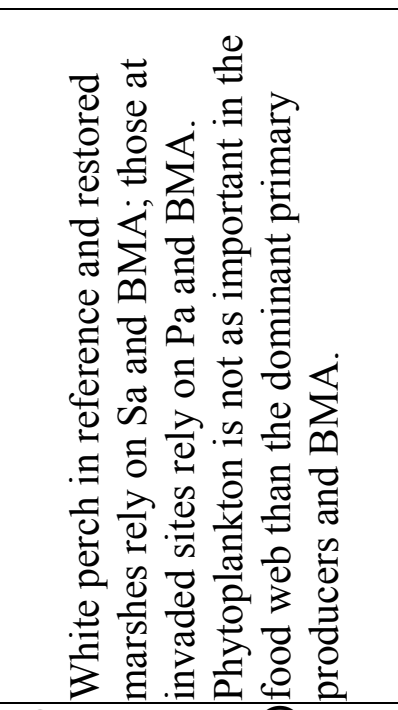 \\
\hline 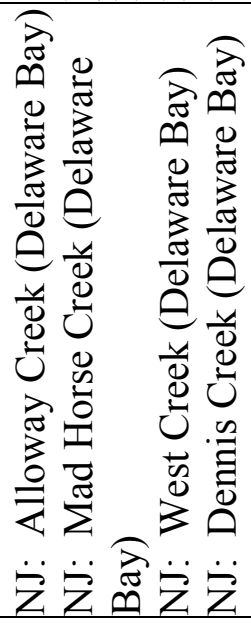 & 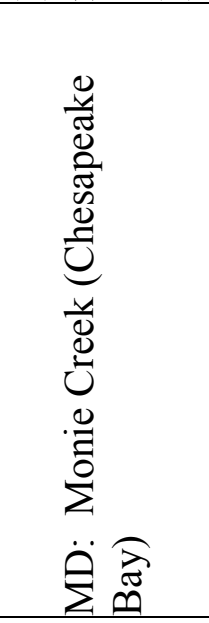 & 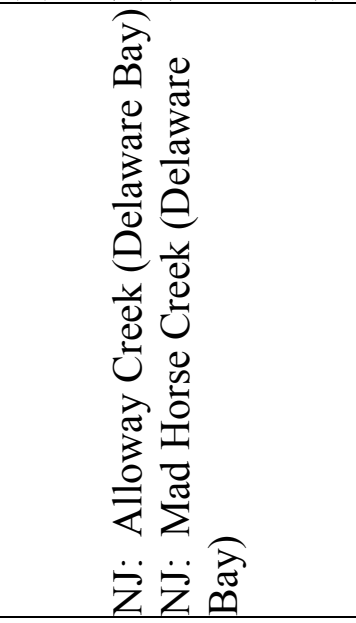 & 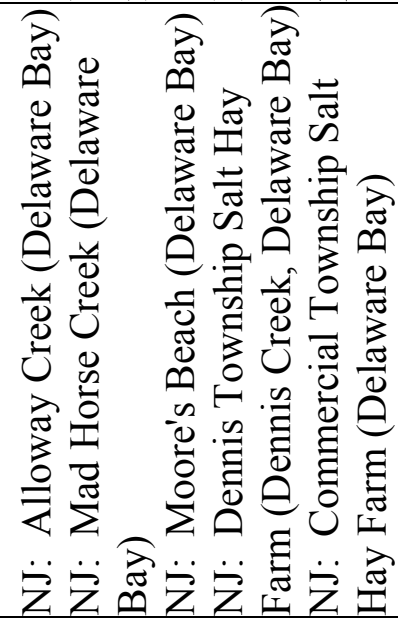 \\
\hline$\vec{z}$ & $\bar{z}$ & $\bar{z}$ & $\vec{\nabla}$ \\
\hline 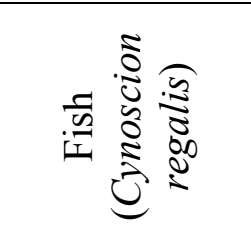 & 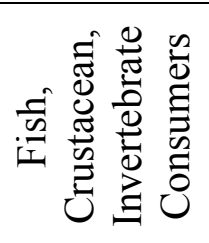 & 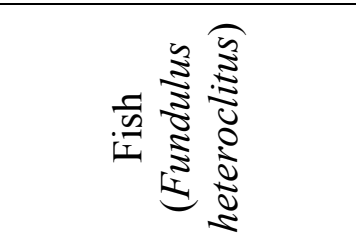 & 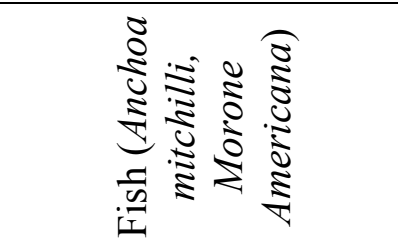 \\
\hline 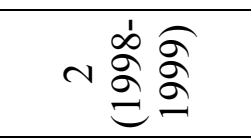 & $-\stackrel{\widehat{̊}}{\stackrel{2}{\varrho}}$ & $-\widehat{\widehat{a}}$ & $-\stackrel{\text { ळे }}{\stackrel{2}{\varrho}}$ \\
\hline 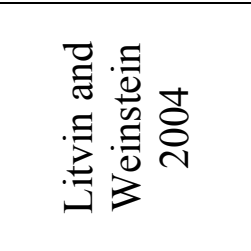 & 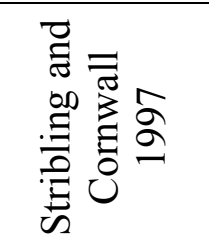 & 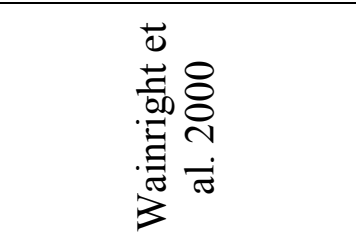 & 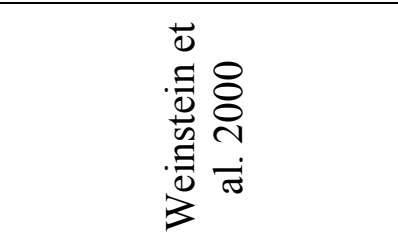 \\
\hline$\stackrel{\infty}{+}$ & g & in & $\bar{n}$ \\
\hline
\end{tabular}


Table 1-A2, cont.

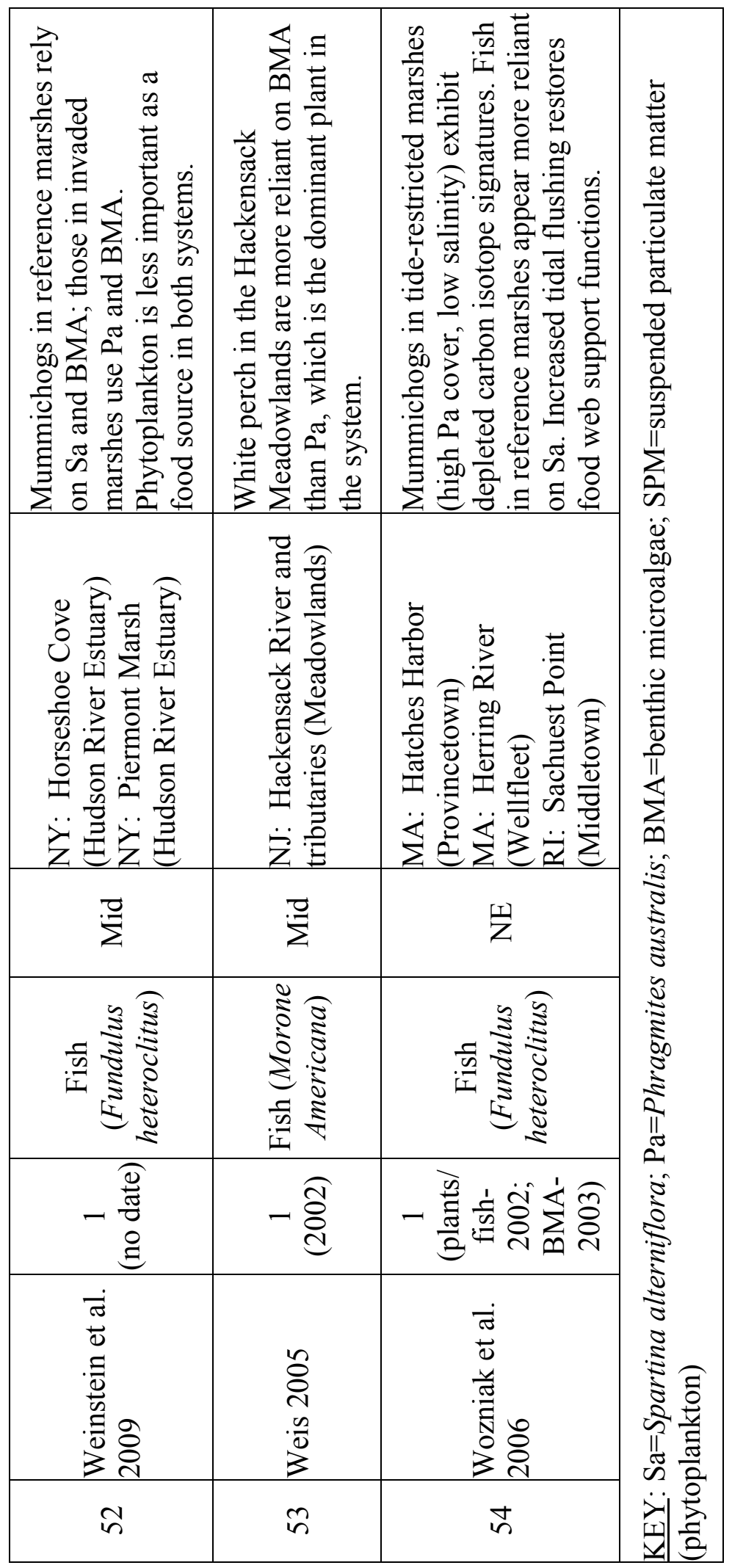


Table 1-A3. Summary of data collection approach for meta-analysis.

\begin{tabular}{|c|c|c|c|}
\hline $\begin{array}{l}\text { Electronic } \\
\text { Databases }\end{array}$ & Search Terms & $\begin{array}{l}\text { Literature } \\
\text { Reviewed }\end{array}$ & Data Gathered \\
\hline 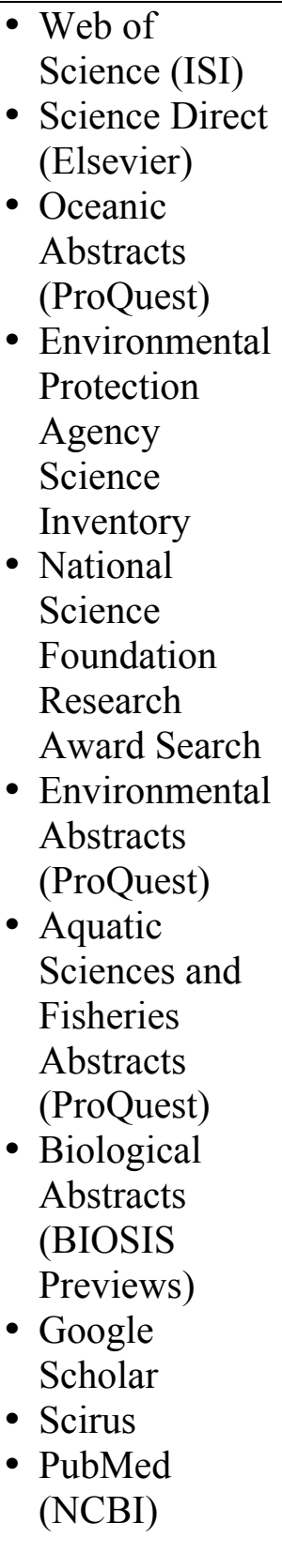 & $\begin{array}{l}\text { - } \text { North } \\
\text { - America } \\
\text { - } \text { Phrantic } \\
\text { - Spartina } \\
\text { - Bird } \\
\text { - Avian } \\
\text { - Fish } \\
\text { - Fundulus } \\
\text { - Nekton } \\
\text { - Invertebrate } \\
\text { - Mollusk } \\
\text { - Reptile } \\
\text { - Amphibian } \\
\text { - Fauna } \\
\text { - Invasion } \\
\text { - Tidally } \\
\text { restricted } \\
\text { - Tidally } \\
\text { - Restored } \\
\text { - Restoration } \\
\text { - Salt marsh } \\
\text { - Wetland } \\
\text { - Estuarine } \\
\text { - Impact } \\
\text { - Non-native } \\
\text { - Invasive } \\
\text { - Food web } \\
\text { - Stable } \\
\text { isotope }\end{array}$ & $\begin{array}{l}\text { - Peer-reviewed } \\
\text { journal articles } \\
\text { - Conference } \\
\text { proceedings } \\
\text { - Government } \\
\text { documents } \\
\text { - Scientific and } \\
\text { technical } \\
\text { reports } \\
\text { - University } \\
\text { dissertation } \\
\text { and master's } \\
\text { theses } \\
\text { - Gray literature } \\
\text { (non- } \\
\text { governmental } \\
\text { organizations) } \\
\text { - Unpublished } \\
\text { studies } \\
\text { (colleagues) }\end{array}$ & $\begin{array}{l}\text { - Species studied } \\
\text { - Taxonomic grouping } \\
\text { - Life history stage } \\
\text { - Response (e.g., } \\
\text { abundance, density, etc.) } \\
\text { - Location of study (region, } \\
\text { GPS point) } \\
\text { - Gear type (quadrat, throw } \\
\text { trap, seine, weir, fyke net, } \\
\text { lift net, breeder trap, pit } \\
\text { trap, core sampler, suction } \\
\text { sampler, flume net, } \\
\text { transects, block net, otter } \\
\text { trawl, visual point counts, } \\
\text { laboratory experiments) } \\
\text { - Marsh habitat (tidal creek, } \\
\text { marsh surface) } \\
\text { - Years of study/field } \\
\text { collections } \\
\text { - Type of system (open, } \\
\text { restricted) } \\
\text { - Type of restoration } \\
\text { (hydrologic, conventional) } \\
\text { - Quantitative data: means } \\
\text { (sample size, standard } \\
\text { deviation, standard error, } \\
\text { if available) } \\
\text { - Environmental data } \\
\text { (salinity, temperature, } \\
\text { dissolved oxygen) } \\
\text { - Auble isotope data/results } \\
\text { habitat impacts } \\
\text { - Comments on study } \\
\text { quality }\end{array}$ \\
\hline
\end{tabular}


Table 1-A4. Criteria used to minimize bias caused by non-independence of data and to group data by response variable.

\begin{tabular}{|c|c|}
\hline Category & $\begin{array}{l}\text { General Rules for Combining Effect Sizes and Grouping Data by } \\
\text { Response Variable }\end{array}$ \\
\hline $\begin{array}{l}\text { Effect Size } \\
\text { (general) }\end{array}$ & $\begin{array}{l}\text { Multiple years of data: We averaged the log response ratios } \\
\text { from each individual year and reported the effect size as a mean } \\
\text { for the entire study. } \\
\text { 'Zero' abundance reported: We did a sensitivity analysis and } \\
\text { added the values } 1,0.1,0.01,0.001 \text {, and } 0.0001 \text { to determine the } \\
\text { smallest number that could be added to all mean data points to } \\
\text { ensure effect ratios could be calculated for all interactions. The } \\
\text { values } 0.001 \text { and } 0.0001 \text { had the least influence on effect ratios; } \\
\text { therefore, } 0.0001 \text { (being the smallest value) was added to all } \\
\text { experimental and control means prior to effect size calculation } \\
\text { (Molloy et al. } 2008 \text {; Kalies et al. } 2010) \text {. } \\
\text { Pre- and post-restoration data: Many authors compared pre- } \\
\text { and post-restoration data for a particular marsh system to a } \\
\text { nearby reference marsh. Therefore, a study can contribute data } \\
\text { to both the 'invaded' and 'restored' meta-analyses [effect sizes } \\
\text { were calculated for pre-restoration data (invaded vs. reference) } \\
\text { and post-restoration data (restored vs. reference)]. These studies } \\
\text { are indicated as 'I, R" under the "marsh studied" column } \\
\text { (Online Resource } 1 \text {, Table } 1 \text {-A1). } \\
\text { Weighted effect size: To calculate Hedges' } d \text {, we needed } \\
\text { estimates of standard deviation and the sample size. If the } \\
\text { authors gave standard error, we calculated standard deviation } \\
\text { based on the error and sample size. If only the sample size was } \\
\text { provided, we calculated } w_{i} \text { by the following equation: } w_{i}=\left(\mathrm{N}_{\mathrm{T}}\right. \\
\left.\mathrm{x} \mathrm{N}_{\mathrm{C}}\right) \text { /( } \mathrm{N}_{\mathrm{T}}+\mathrm{N}_{\mathrm{C}} \text { ), where } \mathrm{N}_{\mathrm{T}} \text { is the treatment sample size and } \mathrm{N}_{\mathrm{C}} \\
\text { is the control sample size (Hedges and Olkin } 1985) \text {. } \\
\text { Data acquisition: If the authors only represented data } \\
\text { graphically, we estimated sampling parameters (i.e., means, } \\
\text { standard deviation, standard error) from figures published in } \\
\text { their studies. }\end{array}$ \\
\hline Metric & $\begin{array}{l}\text { 6) Multiple gear types: If many types of gear were used within the } \\
\text { same study to assess the same impact on fauna (i.e., throw traps } \\
\text { and lift nets used to assess density of adult nekton), then the } \\
\text { mean of those effect ratios was calculated. } \\
\text { Response variables within metric: We pooled the mean within- } \\
\text { study effect sizes as follows: } \\
\text { a. Quantity: Abundance (total viewed/captured), density, } \\
\text { catch-per-unit-effort } \\
\text { b. Condition: Fulton's K, free fatty } \\
\text { acid/triacylglycerol/energy reserves, weight gain, } \\
\text { biomass, length, growth rate, gut fullness }\end{array}$ \\
\hline
\end{tabular}




\begin{tabular}{|c|c|c|}
\hline & & $\begin{array}{l}\text { c. Diversity: Species richness (\# species, Jacknife), } \\
\text { diversity (Shannon Wiener), evenness }\end{array}$ \\
\hline Region & 8) & $\begin{array}{l}\text { Study site locations: Data from different locations within the } \\
\text { same study were reported as separate measures (i.e., if the } \\
\text { authors collected paired invaded-reference data at } 2 \text { study sites, } \\
\text { each site was retained as an individual measurement due to } \\
\text { geographic distance). } \\
\text { Regional categories: } \\
\text { a. Mid-Atlantic Study Sites: New York, New Jersey, } \\
\text { Delaware, and Maryland } \\
\text { b. New England Study Sites: Maine, New Hampshire, } \\
\text { Massachusetts, Rhode Island, and Connecticut }\end{array}$ \\
\hline Taxon & 10) & $\begin{array}{l}\text { Species-level data: If multiple species within a particular } \\
\text { taxonomic category were reported in a study, we calculated the } \\
\text { effect size for each species, and then averaged across species for } \\
\text { the taxon-level meta-analysis. If the author reported a total } \\
\text { number for the taxonomic group (i.e., total nekton density), we } \\
\text { used their total faunal estimate rather than averaging effect size } \\
\text { across species. } \\
\text { Taxonomic categories: } \\
\text { a. Nekton: Aquatic fish and swimming invertebrates } \\
\text { (e.g., crustaceans; shrimp and crabs) } \\
\text { b. Birds: All resident and transient species (e.g., long- } \\
\text { legged waders, shorebirds, etc.) } \\
\text { c. Invertebrates: Benthic infauna and marsh surface } \\
\text { fauna (sessile or benthic macroinvertebrates collected } \\
\text { from the marsh surface via suction samplers or coring } \\
\text { devices; prey items) }\end{array}$ \\
\hline $\begin{array}{l}\text { Life } \\
\text { History } \\
\text { Stage }\end{array}$ & $\begin{array}{l}\text { 12) } \\
13)\end{array}$ & $\begin{array}{l}\text { Adult category: Includes all adult individuals in the population } \\
\text { Sub-adult category: Includes eggs, larvae, 'recruits' (fish), and } \\
\text { juveniles }\end{array}$ \\
\hline $\begin{array}{l}\text { Marsh } \\
\text { Habitat }\end{array}$ & 14) & $\begin{array}{l}\text { Marsh surface: Includes data from pools, measurements taken } \\
\text { on flood tide on the marsh surface, and invertebrate data from } \\
\text { suction samplers and coring devices } \\
\text { Tidal creek: Includes data from intertidal and/or sub-tidal } \\
\text { creeks, including mosquito ditches }\end{array}$ \\
\hline $\begin{array}{l}\text { System } \\
\text { Type }\end{array}$ & 16) & $\begin{array}{l}\text { Open: Data from } P \text {. australis-invaded marshes lacking a } \\
\text { tidal/hydrologic restriction } \\
\text { Restricted: Data from tidally restricted marshes (restrictions } \\
\text { include earthen berms, roads, bridges, undersized/failing } \\
\text { culverts, etc.) }\end{array}$ \\
\hline $\begin{array}{l}\text { Restoration } \\
\text { Type }\end{array}$ & 18) & $\begin{array}{l}\text { Hydrologic: Use of techniques to increase tidal flushing to } \\
\text { decrease extent and cover of } P \text {. australis (replacement of } \\
\text { culvert, construction of bridge, breach of impoundment, etc.) } \\
\text { Conventional: Use of manual/physical (cutting/burning), and } \\
\text { chemical (herbicide) mechanisms to control P. australis }\end{array}$ \\
\hline
\end{tabular}




\section{CHAPTER 2}

The importance of an invasive plant in salt marsh food webs

by

Kimberly L. Dibble ${ }^{1}$; Laura A. Meyerson ${ }^{1,2}$

is submitted to the Journal of Ecology

${ }^{1}$ Department of Natural Resources Science, University of Rhode Island, 1 Greenhouse Road, Kingston, RI 02881.

${ }^{2}$ Institute of Botany, Department of Invasion Ecology, Academy of Sciences of the Czech Republic, Průhonice, CZ 252 43, Czech Republic. 


\section{Summary}

1. We measured the effects of a plant invasion (Phragmites australis) on higher trophic levels in salt marshes in eastern North America by assessing habitat quality at the food web base and by quantifying the importance of primary producers to secondary production using a recently developed Bayesian mixing model (Stable Isotope Analysis in R, "SIAR”).

2. Spartina alterniflora, the dominant native plant in Atlantic coast salt marshes, exhibited significantly greater leaf toughness and higher $\mathrm{C}: \mathrm{N}$ ratios relative to $P$. australis. Benthic microalgae (BMA) and suspended particulate matter (SPM, a proxy for phytoplankton) exhibited the lowest $\mathrm{C}: \mathrm{N}$ indicating higher diet quality. Phenolic concentrations were higher in $P$. australis than $S$. alterniflora but not significantly.

3. We tested the sensitivity of SIAR to variation in discrimination factors $\left(\Delta^{13} \mathrm{C}\right.$, $\Delta^{15} \mathrm{~N}, \Delta^{34} \mathrm{~S}$ ) taken from published literature and found the estimated contributions of primary producers were significantly affected by variation in $\Delta^{13} \mathrm{C}, \Delta^{15} \mathrm{~N}$, and $\Delta^{34} \mathrm{~S}$.

4. Using species-specific discrimination factors we found that native plants contribute the least to Fundulus heteroclitus tissue in restricted (invaded) and restored systems $(\sim 4 \%, \sim 3 \%$, respectively) while BMA was slightly more important $(\sim 7 \%, \sim 14 \%)$. Our model showed that $P$. australis gained dietary importance in restricted and restored marshes $(\sim 34 \%, \sim 32 \%)$ but SPM contributed the most in both systems $(\sim 55 \%, \sim 51 \%)$. The highest contribution of BMA to fish 
tissue ( $35 \%)$ was in uninvaded marshes adjacent to restored systems, with additional contributions from SPM and native plants ( $48 \%, \sim 17 \%)$.

5. Synthesis. Our analysis revealed that SPM, BMA, and macrophytes contribute to energy flow within salt marsh food webs. In reference marshes, secondary production was supported by SPM and BMA, with a small contribution from native plants. These results are consistent with our leaf toughness and leaf chemistry data, which show that SPM and BMA are more palatable diet items. In restricted marshes primary consumers relied on SPM and less on BMA, resulting in a shift in diet toward invasive plant consumption. This is likely due to increased shading of the marsh surface that decreased BMA biomass. Restoration increased the importance of BMA, indicating a shift in ecological recovery toward the uninvaded state.

Keywords: C:N; discrimination factor; Fundulus heteroclitus; invasion ecology; leaf toughness; mixing model; phenolic concentration; Phragmites australis; SIAR; stable isotopes.

\section{Introduction}

Coastal salt marshes are highly productive ecosystems that provide forage, refuge, and nursery habitat for terrestrial and aquatic species worldwide (Beck et al. 2001). Although the importance of the detritus of native salt marsh plants to the direct support of near and off-shore food webs has been debated for decades (Teal 1962; Darnell 1967; Odum 1968; Nixon 1980; Childers et al. 2000), dietary, behavioral, and 
isotopic evidence indicate that salt marsh organic matter is assimilated by higher trophic levels and is mediated by factors such as geomorphology, tidal attributes, and freshwater input (Deegan et al. 2000). In recent years, the invasion of non-native plant species into coastal salt marshes has generated new questions on how food web support functions change when marshes are colonized by plant invaders (Wainright et al. 2000; Weinstein et al. 2000, 2009; Currin et al. 2003). We investigated the contribution of native and invasive primary producers (macrophytes, benthic microalgae, phytoplankton) to the production of a resident omnivorous fish, Fundulus heteroclitus, and examined the quality of primary producer diets for primary consumers in New England salt marshes.

Introduced Phragmites australis subsp. australis (hereafter, "introduced P. australis") is replacing native Spartina alterniflora along the Atlantic coast of North America (Chambers et al. 1999). The P. australis invasion into Atlantic salt marshes provides an excellent model to assess the importance of native vs. introduced salt marsh vegetation at the food web base as well as examine trophic response to ecological restoration because, like other invasive species, it is highly plastic, adapts to a wide range of environmental conditions, is globally distributed, and acts as ecosystem engineer post-invasion (Chambers et al. 1999; Meyerson et al. 2000, 2009).

Introduced $P$. australis has widely invaded oligohaline to polyhaline salt marshes throughout the mid-Atlantic and New England regions of North America (Chambers et al. 1999; Meyerson et al. 2000, 2009). This invasive macrophyte takes advantage of 
reduced salinity and increased disturbance and nitrogen availability associated with tidal restrictions and coastal development by forming near monocultures that decrease native plant diversity, elevate the marsh surface, and reduce the number of small water-filled marsh pools and depressions (Meyerson et al. 2000, 2009; Able et al. 2003; Rooth et al. 2003). Turnover of the dominant plant species and subsequent alterations in habitat structure have been shown to alter the trophic structure of arthropod communities, with a shift from externally feeding herbivores in $S$. alterniflora to concealed detritivorous feeders in P. australis (Gratton and Denno 2005). This trophic structure shift in feeding guilds may change ecosystem function by decreasing the export of organic material and primary consumers off the marsh surface via predators such as nekton and birds. Therefore, a change in dominant primary producer may have far-reaching effects up the food chain to higher-level consumers.

Research thus far on the contribution of native and invasive primary producers in salt marsh food webs in the mid-Atlantic region has produced mixed results. Using a multiple stable isotope approach, Gratton and Denno (2006) found that in invaded marshes arthropod food webs are most likely fueled by BMA or SPM and not the invasive plant. Arthropod assemblages and dietary sources are indistinguishable in restored and uninvaded marshes, suggesting that S. alterniflora forms the base of the food web (Gratton and Denno 2005, 2006). Other studies concluded that introduced $P$. australis partially supports secondary production for fish such as $F$. heteroclitus, Anchoa mitchilli, Cynoscion regalis, and Morone americana (Wainright et al. 2000; Weinstein et al. 2000, 2009; Litvin and Weinstein 2003, 2004). In New England, 
Wozniak et al. (2006) used the carbon isotopic signature $\left(\delta^{13} \mathrm{C}\right)$ of $S$. alterniflora $\left(\mathrm{C}_{4}\right)$ and $P$. australis $\left(\mathrm{C}_{3}\right)$ to trace the flow of organic matter to consumers in tidally restricted, restored, and reference marshes. They found that secondary consumers $(F$. heteroclitus) have depleted carbon signatures in tidally restricted salt marshes invaded by $P$. australis relative to adjacent unrestricted marshes. The $\delta^{13} \mathrm{C}$ in fish tissue became more enriched (i.e., closer to the signature of $S$. alterniflora) as $P$. australis cover was reduced by tidal restoration, providing a useful indicator of the trajectory of ecological change. Therefore, the incorporation of $P$. australis into salt marsh food webs varies by species, study site, and environmental factors, but stable isotopes can be useful in the detection of restoration success.

We investigated whether food web support functions change when invasive plants replace native vegetation in New England and determined how restoration influences the assimilation and transfer of organic matter to higher trophic levels. To do this we asked the following questions: (a) Is there a detectable difference in diet quality of the dominant primary producers in New England salt marshes?; (b) What is the relative importance of macrophytes, benthic microalgae, and phytoplankton in driving secondary production in invaded tidally restricted, restored, and reference salt marshes in New England?; and (c) How does the use of different discrimination factors from published literature affect the outcome and interpretation of results from stable isotope mixing models? 
To address the above questions, we examined diet quality using data on leaf toughness, the C:N ratio, and phenolic concentrations in plant leaves. We used a recently developed Bayesian mixing model in the software package SIAR (Stable Isotope Analysis in R; Jackson et al. 2009; Parnell et al. 2010) to assess the importance of native and invasive primary producers to $F$. heteroclitus production. SIAR represents an improvement over previous modeling approaches because it incorporates discrimination factors (i.e., the change in isotope ratio from prey to consumer tissue, $\Delta{ }^{13} \mathrm{C}, \Delta{ }^{15} \mathrm{~N}, \Delta{ }^{34} \mathrm{~S}$ ) and their variation, the variation in isotopic signatures of each prey source, and source elemental concentrations into the model. To date, most studies using isotopic mixing models have assessed the proportions of direct prey items to the tissue sampled (one trophic level). Instead, we estimated the contribution of primary producers to the production of $F$. heteroclitus that resides approximately two trophic levels above primary producers (Currin et al. 1995, 2003; Deegan and Garritt 1997; Wainright et al. 2000), an approach that has been taken for other species in coastal salt marshes.

Brittain et al. (2012) used SIAR to model the contribution of salt marsh vs. terrestrial primary producer sources to passerine food webs in Sapelo Island, Georgia using discrimination factors to estimate dietary proportions 1.5 to 3 trophic levels above the food web base. In the mid-Atlantic region, Wainright et al. (2000) used a two-source mixing model to estimate a two trophic level shift between juvenile $F$. heteroclitus and baseline diet, doubling the discrimination factors for carbon (1\%o) and assuming a $0 \%$ o shift for sulfur based on values from Peterson and Fry (1987). They estimated that $F$. 
heteroclitus production was supported by $\sim 39 \%$ S. alterniflora in reference marshes (presumably through detrital pathways) and $\sim 73 \%$ P. australis in invaded marshes, with additional contributions from SPM and BMA (Wainright et al. 2000). Therefore, although we do not have data on the isotopic signatures of invertebrate prey at our study sites, we can estimate relative proportions of primary producers supporting the food web using Bayesian mixing models and discrimination factor inputs.

\section{Materials and Methods}

Study Sites and Sampling Locations

We selected four tidally restricted (hereafter, "restricted") and four tidally restored ("restored") salt marshes invaded by introduced $P$. australis along the Atlantic coast of North America (Fig. 2-1). Each restricted or restored site was paired with an adjacent downstream, unrestricted control ("reference") site that was sampled on the same day $(\mathrm{n}=16$ marshes total; 4 restricted, 4 restored, 8 reference). At tidally restricted sites, introduced P. australis was the dominant macrophyte, while the reference sites were primarily colonized by Spartina spp. and other native plants (e.g., Distichlis spicata, Juncus gerardii, Limonium nashii, etc.). At restored sites, a mixture of introduced $P$. australis and Spartina spp. dominated, while reference sites were composed of Spartina spp. and other native plants.

\section{Field Collections}

Three stations were randomly selected a priori within each of the 16 marshes $(\mathrm{n}=48$ experimental units, “EU's"). Because we employed a matched pairs experimental 
design, stations from the restricted marshes were only compared to stations from the adjacent unrestricted (reference) marshes, and stations from the restored marshes were only compared to those from adjacent reference marshes. We collected samples from one of our study sites (Drakes Island, ME) in summer 2009 (7/10/09, 7/22/09) and fall $2009(9 / 1 / 09)$ as part of a preliminary research study and collected samples from all 16 marsh sites in summer $2010(7 / 12-7 / 25 / 10,7 / 29 / 10)$, fall $2010(9 / 22-10 / 3 / 10)$, summer $2011(7 / 11-7 / 23 / 11)$, and fall $2011(9 / 25-10 / 7 / 11)$. We collected water quality data (salinity, temperature, dissolved oxygen) using a YSI-85 (2009-2010) and a YSI Pro2030 (2011) at each station on every sample date with the exception of our four northern sites in Massachusetts and Maine in summer 2010 due to equipment malfunction (K.L. Dibble, unpublished data).

\section{Primary Producers}

We collected the standing live leaves of the dominant plants present at each station $(P$. australis, S. alterniflora, S. patens, D. spicata). Plant dominance was determined via visual inspection of the marsh surface and surrounding area; any plant species representing $>25 \%$ (approximate) cover was selected. For consistency, we randomly selected a minimum of five leaves from the top $15 \mathrm{~cm}$ of the stem from each dominant species and pooled the samples in the field (Wainright et al. 2000; Weinstein et al. 2000; Wozniak et al. 2006). In the lab we rinsed leaves with DI water and froze, freeze-dried, ground, and stored the samples in a $-80^{\circ} \mathrm{C}$ freezer. We sampled plants in 2009 at Drakes Island, at all sites in 2010, and at three sites in 2011 (for clarification of 2010 data). In fall 2011 we collected data on leaf toughness using a handheld leaf 
penetrometer (Chatillon Push/Pull Gauge, Model 719) to test hypotheses on diet quality. At each station where introduced P. australis and/or S. alterniflora were present, we sampled 10 leaves from randomly selected plants and measured the amount of pressure (lbs) needed to penetrate each leaf.

In summer and fall 2010 we collected a 1-liter bottle of water from the top $5 \mathrm{~cm}$ of the water column at each station using amber HDPE bottles and brought them back to the laboratory on ice. In the lab, we vacuum filtered the water through Whatman binderfree glass microfiber filters $(\mathrm{GF} / \mathrm{F} ; 4.7 \mathrm{~cm})$ to collect suspended particulate matter (SPM). Filters were dried in a $40^{\circ} \mathrm{C}$ oven and pelleted for stable isotope analysis. Using scintillation vials, we collected $40 \mathrm{~mL}$ of water from the top $5 \mathrm{~cm}$ of the water column and preserved it with sodium azide to examine dissolved inorganic carbon (DI $\delta^{13} \mathrm{C}$ ) in the water column and to determine whether the water contained a phytoplankton signature (Chanton and Lewis 1999; Wainright et al. 2000; Fry 2002).

We sampled the benthic microalgal (BMA) community using a modification of the Couch (1989) method, whereby the top $1 \mathrm{~cm}$ of bare mudflat, sandflat, or sediment between vegetation at low tide was collected and brought on ice in the dark to the laboratory. We induced microalgal vertical migration in our greenhouse at the University of Rhode Island Greene H. Gardner Crops Research Center by spreading the sediment in trays, covering the wet sediment with a thin layer of Acros Organics precombusted silica sand, covering the sand with Nitex mesh, and then covering the mesh with another layer of sand. Window screening mounted on Styrofoam was 
placed on top of the sand to shade the surface. After 12 hours of daylight, we removed the mesh, rinsed BMA off the mesh with DI water, removed inorganic sediment, and vacuum pumped the water through Whatman GF/F filters $(4.7 \mathrm{~cm}$; Wainright et al. 2000; Wozniak et al. 2006). Filters were dried in a $40^{\circ} \mathrm{C}$ oven and pelleted for stable isotope analysis.

Fish

At each station we deployed two Frabill vinyl-coated round minnow traps on flood tide for 30 minutes, combined the contents from both traps, then randomly selected five male and five female adult fish $(>40 \mathrm{~mm})$ representing the largest, smallest, and middle size ranges of fish available. We measured (fork length, mm), weighed (centigrams), and then humanely euthanized the fish via IACUC-sanctioned procedures (URI Protocol \#AN-09-05-020). In the laboratory we removed fish digestive tracts and regurgitated food to ensure the isotopic signature encompassed assimilated food only. We rinsed fish in DI water and then froze, freeze-dried, ground (whole-body), and stored the fish in a $-80^{\circ} \mathrm{C}$ freezer. Due to delayed spawning in the Northeast in 2011 we captured large quantities of gravid fish, providing the opportunity to assess nutrient allocation to reproduction (i.e., whether nutrients from different food sources are preferentially allocated to egg development; O’Brien et al. 2000). We collected the egg sacks from female body cavities, combined the eggs from each station into one sample, and prepared them for stable isotope analysis as we did with the fish and plants above. 


\section{Laboratory Analyses}

Inorganic Carbon Removal

Carbon incorporated into tissues and carbon in inorganic carbonate originates from different sources and can bias stable isotope results, so it has been common practice to remove inorganic carbon from field samples prior to analysis (Fry 1988; Cloern et al. 2002; Jacob et al. 2005). However, inorganic carbon removal via acidification can affect the $\delta^{15} \mathrm{~N}$ signature of the sample (Pinnegar and Polunin 1999; Jacob et al. 2005), so we conducted a small experiment using plant, fish, BMA, and SPM samples from 2009 field collections to determine whether it was necessary to acidify our samples prior to isotope analysis. Subsamples were treated with dilute (10\%) hydrochloric acid, dried in a $40^{\circ} \mathrm{C}$ oven (without rinsing, to minimize loss of dissolved organic matter), re-ground with a mortar and pestle, and weighed on a microbalance into Costech tin capsules $(3.5 \times 5 \mathrm{~mm})$ to the nearest $0.001 \mathrm{mg}$ (Fry 1988; Cloern et al. 2002). We ran the acidified samples for $\delta^{13} \mathrm{C}$ using an isotope ratio mass spectrometer at the Environmental Protection Agency, Atlantic Ecology Laboratory in Narragansett, Rhode Island and compared the results to data from the unacidified parent sample.

\section{Lipid Removal}

To avoid potential error due to differential fractionation of $\delta^{13} \mathrm{C}$ in lipids, carbohydrates, and proteins during tissue synthesis (Post et al. 2007), we removed lipids from individually ground fish samples. Briefly, powdered samples were packed into Whatman cellulose extraction thimbles and washed with a non-polar lipid solvent (petroleum ether) for six hours using a Soxhlet apparatus (Dobush et al. 1985). After 
lipid extraction, we composited ten fish from each EU using a mortar and pestle and weighed three replicates to the nearest $0.001 \mathrm{mg}$ into tin capsules for isotope analysis (Fry et al. 2008). We did not remove lipids from ground eggs due to the limited quantity of material available; instead, we corrected the $\delta^{13} \mathrm{C}$ values using an equation based on the relationship between lipid content, $\mathrm{C}: \mathrm{N}$ ratio, and $\delta^{13} \mathrm{C}$ (Eqn. 3; Post et al. 2007).

Stable Isotope, Elemental, and Phenolic Compound Analyses We determined the isotopic composition $\left(\delta^{13} \mathrm{C}, \delta^{15} \mathrm{~N}\right)$ and elemental composition $(\% \mathrm{C}$, $\% \mathrm{~N}$ ) of fish, eggs, plants, SPM, and BMA using an Elementar Vario Micro Cube elemental analyzer interfaced to an IsoPrime 100 mass spectrometer (precision $= \pm 0.2 \%$ ) at the EPA Atlantic Ecology Laboratory. The $\mathrm{C}$ and $\mathrm{N}$ isotopic composition is expressed as a part per thousand (per mill, \%) deviation from the reference standard for carbon (PDB) and from the composition of $\mathrm{N}_{2}$ in the air and was calculated using the following equation:

$$
\delta \mathrm{X}=\left[\left(R_{\text {sample }} / R_{\text {standard }}\right)-1\right] \times 1000
$$

Where $\mathrm{X}$ is ${ }^{13} \mathrm{C}$ or ${ }^{15} \mathrm{~N}$ and $\mathrm{R}$ is the ratio of ${ }^{13} \mathrm{C} /{ }^{12} \mathrm{C}$ and ${ }^{15} \mathrm{~N} /{ }^{14} \mathrm{~N}$. Samples were randomly run in batches of 80-100 due to instrument capacity. Laboratory standards (blue mussel) were placed in duplicate every $20^{\text {th }}$ sample and at the beginning and end of each run to check and correct for instrument drift. We analyzed $10 \%$ of the primary producer samples in duplicate; composited samples of fish and eggs were run in triplicate (Fry et al. 2008). The mean of the duplicate and triplicate samples for each EU/time period was used for statistical analyses to avoid pseudoreplication. Ground 
samples of fish, eggs, and primary producers were packaged with vanadium pentoxide and analyzed by Iso-Analytical in Crewe, United Kingdom for $\delta^{34} \mathrm{~S}$ and $\% \mathrm{~S}$, with $10 \%$ run in duplicate.

We sent preserved $40 \mathrm{~mL}$ scintillation vials of water to the University of California Davis Stable Isotope Facility for analysis of DI $\delta^{13} \mathrm{C}$. To examine plant tissue phenolic compounds and verify elemental concentration, we sent ground leaf samples $(S$. alterniflora and $P$. australis) from summer and fall 2010 to the University of Louisiana Agricultural Research and Extension Center, Soil Testing and Plant Analysis Lab in Baton Rouge, Louisiana. Percent carbon and nitrogen were determined using a Leco CN Analyzer. Phenolic compounds were extracted using $50 \%$ methanol, after which a $100 \mu \mathrm{L}$ aliquot of extract was mixed with distilled water, Folins Reagent, and sodium carbonate. Absorbance was read at a wavelenth of 720nm. Samples were run in duplicate; if replicate absorbance values were not within 0.020 ODU the replicate was re-analyzed. The phenolics concentration was not broken down into its constituent components; however, the standard curve used to calculate the concentration was constructed using gallic acid (a phenolic compound in plants).

\section{Data Analysis}

We analyzed data using SAS (v. 9.2) and the R Statistical Environment (v. 2.15.0); figures were developed using SigmaPlot (v. 9.0). Assumptions of normality and equality of variances within datasets were verified prior to all statistical analyses. We examined whether it was necessary to remove inorganic carbon from samples using a 
two-sample paired t-test of means. Regression was used to examine the relationship between salinity, SPM $\delta^{13} \mathrm{C}$, and water column DI $\delta^{13} \mathrm{C}$. For leaf toughness data collected in fall 2011, we summarized 10 pressure measurements from each EU prior to analysis. Due to violations of normality and homogeneity of variance, we analyzed leaf toughness and phenolics data from S. alterniflora and P. australis using Welch's t-tests. An independent samples t-test was used to examine differences in C:N of $S$. alterniflora and P. australis. Because we collected samples from each EU over time, we analyzed differences in mean $\delta^{13} \mathrm{C}, \delta^{15} \mathrm{~N}, \delta^{34} \mathrm{~S}, \% \mathrm{C}, \% \mathrm{~N}$, and $\% \mathrm{~S}$ in fish tissue using repeated measures mixed model Analysis of Variance (ANOVA). We used SLICES in the model to examine interaction effects to determine whether there were significant differences in the response after explanatory variables were incorporated into the model (i.e., marsh type, time, region); mean fish length was used as a covariate to account for trophic level differences associated with $\delta^{15} \mathrm{~N}$. We used Heterogeneous Autoregressive (1) as our covariance structure because it assumes that data that are farther apart in time will be less similar and that each time period has its own unique variance.

We used mixing models in SIAR to investigate the contribution of dominant primary producers to the food web base (Jackson et al. 2009; Parnell et al. 2010). In SIAR, we ran 200,000 iterations with an initial discard of 50,000, thinned by 15 , resulting in 10,000 posterior draws. When SIAR has difficulty differentiating between possible sources the posterior samples for the source contributions to diet are likely to be highly negatively correlated, so we assessed model fit by examining the highest 
correlations between sources in each model. Because the use of discrimination factors can influence model output (Bond and Diamond 2011), we ran a sensitivity analysis using $\Delta \Delta^{13} \mathrm{C}, \Delta{ }^{15} \mathrm{~N}$, and $\Delta^{34} \mathrm{~S}$ values reported from the literature. Adult $F$. heteroclitus $(>40 \mathrm{~mm})$ are omnivorous and have been estimated at two trophic levels above primary producers (Currin et al. 1995, 2003; Deegan and Garritt 1997; Wainright et al. 2000), so discrimination factors and errors were doubled to account for tissue to diet discrimination between primary consumers (invertebrates) and their diet (primary producers) and secondary consumers (F. heteroclitus) and their diet (invertebrates/primary producers). We believe this is an appropriate approach because the range of discrimination factors used to correct fish to omnivorous prey diets $\left(\Delta^{13} \mathrm{C}\right.$ : $\left.0.40-3.36 ; \Delta^{15} \mathrm{~N}: 2.30-3.73\right)$ are similar to those used to correct invertebrate to primary producer diets $\left(\Delta^{13} \mathrm{C}:-0.50-2.50 ; \Delta^{15} \mathrm{~N}: 1.50-2.70\right.$; Gratton and Denno 2006; Caut et al. 2009; Brittain et al. 2012).

We used six sets of discrimination factors, five of which were reported directly in the literature (Fry 1988; Post 2002; McCutchan et al. 2003; Dennis et al. 2010; Elsdon et al. 2010) and the sixth represents the median value of $\Delta{ }^{13} \mathrm{C}, \Delta{ }^{15} \mathrm{~N}$, and $\Delta \Delta^{34} \mathrm{~S}$ compiled from the above literature and from other publications (Peterson and Fry 1987; Vanderklift and Ponsard 2003; Caut et al. 2009; Wyatt et al. 2010; termed "crossstudy $\Delta$ values"). Discrimination factors from Fry (1988), Post (2002), McCutchan et al. (2003), and Dennis et al. (2010) were experimentally determined primarily using fish and aquatic organisms and differ based on trophic level, species, tissue sampled, diet fed, and sample preparation methodology. Elsdon et al. (2010) experimentally 
determined discrimination factors for $F$. heteroclitus fed a range of diets; however, we selected the discrimination factors that closely resembled our sample methodology (i.e., lipid-free, non-acidified $F$. heteroclitus muscle tissue from fish fed an omnivorous diet). Source concentration dependence values were not entered into SIAR because the incorporation of Whatman $\mathrm{GF} / \mathrm{F}$ filter weight into $\% \mathrm{C}, \% \mathrm{~N}$, and $\% \mathrm{~S}$ led to lower (and erroneous) elemental concentrations for BMA and SPM in our data set.

\section{Results}

\section{Basal Diet Quality}

We detected significant differences in the toughness of S. alterniflora and P. australis leaves ( $\mathrm{p}<0.0001, \mathrm{t}_{45.91}=-10.66$ ), with the former requiring significantly more pressure to penetrate $(\bar{x}=3.24 \pm 0.53 \mathrm{lbs} ; \mathrm{n}=47)$ than the latter $(\bar{x}=1.88 \pm 0.47 \mathrm{lbs} ; \mathrm{n}=22)$. The C:N ratio generated via mass spectrometry at the EPA laboratory mirrored results from LSU, so we used the latter dataset for analysis of vascular plant C:N. Mirroring leaf toughness results, S. alterniflora had a significantly higher ratio of carbon to nitrogen $(\bar{x}=21.05 \pm 6.12 ; \mathrm{n}=16)$ than $P$. australis $(\bar{x}=16.29 \pm 3.48 ; \mathrm{n}=17$; $\left.\mathrm{p}=0.0095, \mathrm{t}_{31}=-2.76\right)$. The mean C:N ratios of BMA $(\bar{x}=8.84 \pm 2.29 ; \mathrm{n}=33)$ and SPM $(\bar{x}=6.95 \pm 0.92 ; \mathrm{n}=108)$ were lower than live vascular plants. Using the leaf samples measured for C:N, we found that the concentration of phenolic compounds in $P$. australis was higher $(\bar{x}=109.10 \pm 38.73 \mu \mathrm{M} / \mathrm{g} ; \mathrm{n}=17)$ than S. alterniflora $(\bar{x}=91.41$ $\pm 12.72 \mu \mathrm{M} / \mathrm{g} ; \mathrm{n}=16$ ), but the difference between plant species was not significant $\left(\mathrm{p}=0.0816 ; \mathrm{t}_{21.02}=1.83\right)$. 


\section{Stable Isotope Analysis}

Primary Producers

We examined the relationships between DI $\delta^{13} \mathrm{C}$, salinity, and SPM $\delta^{13} \mathrm{C}$ to determine whether SPM samples contained a phytoplankton signature and whether that signature was affected by changes in salinity between marsh types. Phytoplankton fix water column dissolved inorganic carbon and fractionate that carbon upon uptake, resulting in SPM values that are depleted in $\delta^{13} \mathrm{C}$ by $\sim 20 \%$ (Chanton and Lewis 1999; Fry 2002). Our data agree with this estimate, as SPM $\delta^{13} \mathrm{C}$ was depleted by $\sim 18 \%$ relative to DI $\delta^{13} \mathrm{C}$ (Fig. 2-2). The mean $\mathrm{C}: \mathrm{N}$ ratio of SPM was $6.9-7.0$ at our stations, also indicating that seston collected on filters was of phytoplankton origin (Redfield 1958). We found significant positive relationships between salinity and both DI $\delta^{13} \mathrm{C}$ $\left(r^{2}=0.81, p<0.0001 ; n=62\right)$ and $\operatorname{SPM} \delta^{13} \mathrm{C}\left(\mathrm{r}^{2}=0.34, \mathrm{p}<0.0001 ; \mathrm{n}=62 ;\right.$ Fig. $\left.2-2\right)$, indicating the carbon isotopic composition of SPM is not fixed as it can be with plants; rather, it changes with salinity. Phytoplankton values are strongly influenced by changes to DI $\delta^{13} \mathrm{C}$ (and hence, salinity) in the water column $\left(\mathrm{r}^{2}=0.52, \mathrm{p}<0.0001\right.$; n=93; Fig. 2-3) so we ran separate models for each of the four marsh types to reduce error associated with local environmental conditions.

Removal of inorganic carbon from primary producer and consumer samples using $10 \%$ hydrochloric acid did not significantly affect $\delta^{13} \mathrm{C}\left(\mathrm{p}=0.0935 ; \mathrm{t}_{36}=-1.72\right)$, so we did not remove inorganic carbon from samples used in our primary analysis. The mean isotopic composition, elemental concentration, and $\mathrm{C}: \mathrm{N}$ ratio of primary producer sources are reported in Table 2-1. The similarity in isotopic composition of the 
dominant salt marsh grasses $S$. alterniflora, S. patens, and D. spicata necessitated the combination of these three sources into one native 'salt marsh plant' source for use in SIAR. Overall, mean $\delta^{13} \mathrm{C}$ was distinct for the salt marsh plant $(\bar{x}=-14.34 \pm 0.57)$, BMA $(\bar{x}=-19.74 \pm 2.98), \operatorname{SPM}(\bar{x}=-21.10 \pm 2.69)$, and P. australis $(\bar{x}=-26.12 \pm$ 1.20) sources. Mean $\delta^{15} \mathrm{~N}$ was distinct for $\operatorname{SPM}(\bar{x}=3.72 \pm 3.06)$ but indistinguishable between salt marsh plants $(\bar{x}=6.07 \pm 2.09)$, BMA $(\bar{x}=7.17 \pm$ 4.06), and $P$. australis $(\bar{x}=6.68 \pm 1.90)$. Sulfur isotopes were highly variable but distinguished BMA $(\bar{x}=-3.51 \pm 10.41)$ and SPM $(\bar{x}=13.71 \pm 6.09)$ from salt marsh plants $(\bar{x}=3.94 \pm 9.82)$ and $P$. australis $(\bar{x}=2.35 \pm 9.52)$. Stable isotope biplots show the relationship between the four dominant sources in restricted, restored, and reference marsh food webs as well as their relationship to secondary consumers $(F$. heteroclitus) in the system (Figs 2-4a,b; 2-5a,b).

Fish

We captured 1,920 adult $F$. heteroclitus from 48 stations in the summer and fall seasons from 2010-2011, totaling 192 composited lipid-free whole-body samples. We found significant differences in the isotopic signature of fish inhabiting tidally restricted vs. reference marsh sites for $\delta^{13} \mathrm{C}\left(\mathrm{p}<0.0001, \mathrm{t}_{40}=7.83\right)$ and $\delta^{15} \mathrm{~N}(\mathrm{p}=0.0366$, $\left.\mathrm{t}_{40}=-2.16\right)$ but not for $\delta^{34} \mathrm{~S}, \% \mathrm{C}, \% \mathrm{~N}$, or $\% \mathrm{~S}(\mathrm{p}>0.05$; Table $2-1)$. Results for differences between the restricted and reference systems for $\delta^{13} \mathrm{C}$ were significant and in agreement in the Long Island Sound (LIS) and Gulf of Maine (GOM) regions and in all four time periods sampled $(\mathrm{p}<0.05)$, indicating that restricted and reference marsh fish may be incorporating different sources of carbon in their tissue. However, the 
depleted carbon isotopic signature of fish in restricted marshes relative to those in the reference marshes may simply be due to differences in salinity and changes to DI $\delta^{13} \mathrm{C}$ and SPM $\delta^{13} \mathrm{C}$ as discussed above. The significant difference in fish $\delta^{15} \mathrm{~N}$ signatures in the restricted vs. reference marshes was driven by samples collected in LIS in the summer $\left(\mathrm{p}=0.0510, \mathrm{t}_{119}=-1.97\right)$ and fall of $2010\left(\mathrm{p}=0.0098, \mathrm{t}_{119}=-2.63\right)$. For fish residing in the tidally restored vs. reference marsh sites, we found significant differences in the $\delta^{13} \mathrm{C}\left(\mathrm{p}=0.0370, \mathrm{t}_{40}=2.16\right)$ and $\% \mathrm{~S}\left(\mathrm{p}=0.0358, \mathrm{t}_{40}=2.17\right)$ in fish tissue but not for $\delta^{15} \mathrm{~N}, \delta^{34} \mathrm{~S}, \% \mathrm{C}$, or $\% \mathrm{~N}\left(\mathrm{p}>0.05\right.$; Table 2-1). Overall differences in $\delta^{13} \mathrm{C}$ were driven by differences in carbon source for one time period and region only: summer 2011 in the GOM $\left(\mathrm{p}=0.0291, \mathrm{t}_{119}=2.21\right)$. The isotopic composition of wholebody $F$. heteroclitus was similar to eggs although egg tissue was $\sim 1-1.5 \%$ o depleted in $\delta^{13} \mathrm{C}$, which could be an artifact of differences in methodology used to correct for lipid content (direct lipid removal vs. C:N correction). Overall, this data indicates that fish are allocating similar nutrients to reproduction as they are to maintaining overall growth (Table 2-1). Using the discrimination factors in Table 2-2, F. heteroclitus at our study sites are approximately two trophic levels above primary producers, confirming previous estimates (Currin et al. 1995, 2003; Deegan and Garritt 1997; Wainright et al. 2000; Figs 2-4a,b; 2-5a,b).

\section{Basal Diet Proportions}

SIAR model performance using our data can be considered moderate based on our source correlation coefficients in the range 0.3-0.7 (Bond and Diamond 2011;

Doucette et al. 2012). Sensitivity analysis revealed that using different discrimination 
factors from the literature produce different estimates of source contributions to basal diet (Table 2-2). Salt marsh plant and phytoplankton contributions fluctuated the most, with posterior distributions ranging from $0-71 \%$ and $4-74 \%$ depending on marsh type and $\Delta{ }^{13} \mathrm{C}, \Delta{ }^{15} \mathrm{~N}$, and $\Delta^{34} \mathrm{~S}$ used. Phragmites australis and BMA had a smaller range (0-49\% and $0-54 \%$, respectively). Overall, the median value of SPM source contributions ranged from $20-55 \%$ in restricted marshes and $27-51 \%$ in restored marshes. Introduced $P$. australis ranged in importance from 4-34\% in restricted marshes and $1-32 \%$ in restored marshes. Benthic microalgae was least important in restricted marshes (7-17\%) and had the highest potential source contributions in reference marshes adjacent to restored sites (13-35\%).

Elsdon et al. (2010) experimentally measured $\Delta^{13} \mathrm{C}$ and $\Delta^{15} \mathrm{~N}$ in the non-acidified, lipid-free muscle tissue of $F$. heteroclitus reared on herbivorous, carnivorous, and omnivorous diets in the laboratory. This represents the closest experimentally measured isotopic change from tissue to diet for our study species so we compared model output using Elsdon et al. (2010) discrimination factors for $F$. heteroclitus on an omnivorous diet (Bio-Vita) to results using the median cross-study $\Delta$ values to illustrate the importance of using species-specific discrimination factors. Using Elsdon et al. (2010) we found that BMA contributed the least to basal diet in restricted (median $\sim 7 \%$ ) and restored $(\sim 14 \%)$ marshes, with higher contributions in reference marshes adjacent to the restricted and restored sites ( $\sim 21 \%$ and $\sim 35 \%$, respectively). Introduced $P$. australis contributed $\sim 34 \%$ and $\sim 32 \%$ to basal diet in restricted and restored marshes, respectively, and SPM was equally important in all marshes ( 48- 
$55 \%)$. Salt marsh plants represented a smaller fraction of basal resources in restricted ( $4 \%)$, restored $(\sim 3 \%)$, and reference marshes adjacent to restricted and restored sites ( $\sim 27 \%$ and $\sim 17 \%$, respectively; Table $2-2)$.

The median cross-study $\Delta$ values yielded different results. Benthic microalgae represented a large proportion of the base of the food web in restored and reference marshes $(\sim 19 \%, \sim 19 \%, \sim 21 \%)$, and was least important in restricted marshes $(\sim 14 \%)$. Phragmites australis contributed $\sim 16 \%$ to the base of the food web in restricted marshes and $\sim 4 \%$ to restored marshes. Phytoplankton and salt marsh grasses were equally important in all systems and collectively formed the largest proportions of the food web base ( $\sim 30-40 \%$ each). Reference marshes contained the highest proportions of sources with low C:N ratios (i.e., SPM, BMA) relative to restricted and restored sites (Table 2-2).

\section{Discussion}

\section{Basal Diet Quality}

The flow of energy through salt marsh food webs is partially influenced by the diet quality of primary producers at the base of the food web. The C:N ratio is often used as an indicator of the nutritional value of plant sources, with higher $\% \mathrm{~N}$ values (i.e., lower C:N ratio) indicating a higher quality diet. In our study systems SPM and BMA exhibited the lowest $\mathrm{C}: \mathrm{N}$ and vascular plants exhibited the highest $\mathrm{C}: \mathrm{N}$. Therefore, SPM and BMA are nutritionally superior to vascular plants and are more likely to be preferentially consumed by primary consumers. This is likely due to high palatability 
and the short, efficient nature associated with algal-based food webs leading to higher trophic levels (Deegan et al. 2000). Fish tissue from reference marshes contained high proportions of nutrients from resources exhibiting low $\mathrm{C}: \mathrm{N}$ ratios, indicating that primary consumers target high quality and palatable dietary items. Other studies have found that invertebrates such as amphipods, copepods, isopods, polychaetes, oligochaetes, and snails (the primary diet items of $F$. heteroclitus) preferentially graze on BMA and SPM and can locally reduce BMA biomass by as much as $70 \%$ per day (Currin et al. 2003; Gratton and Denno 2006; Deegan et al. 2007; Galván et al. 2008, 2011). This lends credence to our finding of the importance of these more palatable dietary items in salt marsh food webs, especially for fish using reference marshes.

Similar to our results, Galván et al. $(2008,2011)$ found that Spartina may not be an important basal resource in New England salt marshes. Although macrophytes do play a role in the flow of energy through salt marshes, they have high proportions of structural material typically only made available to consumers through detrital pathways (Deegan et al. 2000). Invertebrates such as the common salt marsh amphipod Gammarus palustris and omnivorous marsh crab Armases cinereum will consume live S. alterniflora but only after the destruction of its toughness by physical or microbial pathways (Pennings et al. 1998; Parker et al. 2008). Our data and those of others show that leaf toughness correlates with C:N (Parker et al. 2008; Jiménez et al. 2012), so the vascular plant detritus most likely to enter into salt marsh food webs will have low leaf toughness and $\mathrm{C}: \mathrm{N}$ ratios. However, invertebrates that consume live 
vascular plants and detritus can be deterred from consumption if the plant contains phenolic compounds such as tannin and gallic acid (Hendricks et al. 2011).

Invasive plants can contain higher concentrations of phenolic compounds relative to native plants (Callaway and Ridenour 2004; Orr et al. 2005), a potential mechanism to deter herbivory (Grosholz 2010; Hendricks et al. 2011). We compared phenolic concentrations in the leaves of the dominant vascular plants and found a lower but (marginally) non-significant concentration in native vs. invasive plant species $(\bar{x}=$ $91.41 \pm 12.72 \mu \mathrm{M} / \mathrm{g}$ vs. $\bar{x}=109.10 \pm 38.73 \mu \mathrm{M} / \mathrm{g} ; \mathrm{p}=0.0816$ ), which could be due to high variability in our data. Hendricks et al. (2011) examined the feeding preferences of a common salt marsh invertebrate, Littoraria irrorata, on the leaves of introduced P. australis and native S. alterniflora and found that the snail consumed 26x more ground S. alterniflora than P. australis even though the native plant was $1.3 \mathrm{x}$ tougher, suggesting a chemical deterrent against herbivory. Although $P$. australis at our study sites exhibited lower C:N and leaf toughness measurements than S. alterniflora, the higher mean phenolic concentration may deter herbivory and its incorporation into the food web via primary consumers, as evidenced by a higher assimilation of SPM into fish tissue. Collectively these results indicate that there is a spectrum of decreasing diet quality from SPM (highest) to BMA to $P$. australis to $S$. alterniflora (lowest).

\section{Basal Diet Proportions}

Use of stable isotope mixing models in salt marsh ecosystems can be difficult due to the presence of multiple plant sources that often have overlapping isotopic signatures 
(Fry and Sherr 1984; Post 2002; Currin et al. 2003). The four dominant sources in our study system were distinguishable by at least one isotope and SIAR model performance was moderate using a multiple stable isotope approach. Our sensitivity analysis using different sets of discrimination factors from the literature (Table 2-2) revealed large variability in SIAR model results, confirming results from Bond and Diamond (2011) on the importance of experimentally determining discrimination factors specific to the study organism for use in isotope mixing models (Martínez del Rio et al. 2009). In our study, use of discrimination factors from Fry (1988), Post (2002), McCutchan et al. (2003), Dennis et al. (2010), and the median cross-study $\Delta$ values yielded similar dietary proportions across all sources (Table 2-2). Speciesspecific discrimination factors from Elsdon et al. (2010) yielded very different results (Table 2-2) but are closer to what would be expected in these study systems based on our research and those of others that indicate that SPM and BMA are important resources at the base of the food web (Kneib et al. 1980; Hughes and Sherr 1983; Deegan et al. 2000).

In New England salt marshes, SPM and BMA support primary consumers but the relative contributions of sources may vary with environmental factors (season, tidal flow, habitat characteristics), feeding mode, and the presence of predators (Galván et al. 2008, 2011). Our results support previous studies in invaded study systems that have linked reductions in BMA to increased shading from $P$. australis (Wainright et al. 2000; Currin et al. 2003; Weis 2005; Bushaw-Newton et al. 2008). We found that the importance of BMA as a food source declined in tidally restricted and restored 
marshes and was highest in uninvaded marshes, where shading by overhanging plants would not be as evident. In our study, the median combined contributions for BMA and SPM ranged from $62-83 \%$ and the median combined contributions for salt marsh and $P$. australis vascular plants ranged from $17-38 \%$. These results demonstrate the importance of algae and plankton at the food web base for invertebrate fauna that support higher trophic levels (Currin et al. 1995; Deegan et al. 2007) and emphasize the importance of preventing plant invasions that reduce the abundance of these dietary resources.

Previous studies that qualitatively analyzed stable isotope data from $F$. heteroclitus and other species in unrestricted $P$. australis-invaded systems in the mid-Atlantic found that, for the most part, fish in uninvaded marshes obtained nutrients from a combination of S. alterniflora, BMA, and to a lesser degree, SPM. Fish in invaded marshes assimilated nutrients from P. australis, BMA, and SPM into their tissue (Wainright et al. 2000; Weinstein et al. 2000, 2009; Litvin and Weinstein 2003, 2004). Currin et al. (2003) found that $F$. heteroclitus in restored marshes rely on a combination of all four sources, with potentially greater reliance on BMA. Our results agree with these findings but reveal a greater reliance on SPM across all marsh types, with contributions from BMA and the dominant macrophyte in each system.

The proportions of nutrients in fish tissue originating from vascular plants at our study sites in New England are lower than those reported in the mid-Atlantic region (e.g., $\sim 39 \%$ S. alterniflora in reference marshes and $\sim 73 \%$ P. australis in invaded marshes; 
Wainright et al. 2000). The median contribution of salt marsh plants at our reference marsh sites was $\sim 27 \%$ while the median contribution of $P$. australis at our restricted marsh sites was $\sim 34 \%$, indicating that although the invasive plant was dominant in the restricted marshes, primary producers within the water column (SPM) formed the majority of the food web base. Restoration of tidal flow increased food web reliance on BMA, likely due to decreased shading of the marsh surface. Benthic microalgal productivity can rival that of Spartina spp. and has long been noted to be an important component of salt marsh food webs readily available to primary consumers that support higher trophic levels (Zedler 1980; Currin et al. 1995, 2003; Galván et al. 2008, 2011). Protecting and restoring salt marsh habitats, decreasing the cover of invasive plants, and maintaining high water quality standards for healthy phytoplankton and benthic microalgal communities are therefore critically important to supporting secondary production in coastal ecosystems.

\section{Acknowledgements}

We thank Rick McKinney at the Environmental Protection Agency (EPA) Atlantic Ecology Laboratory for use of the Isotope Ratio Mass Spectrometer, guidance on preparing samples, and for the interpretation of IRMS output. We thank Peter August and the University of Rhode Island (URI) Environmental Data Center for drafting the map of study sites. Many thanks to Jim Cronin and Warwick Allen at Louisiana State University for leaf chemistry analysis. The following agencies and organizations provided support: EPA STAR Graduate Fellowship (FP-91710001-0), National Oceanic and Atmospheric Administration NERR Graduate Fellowship 
(NA09NOS4200041), National Science Foundation (NSF) IGERT Grant to the

Coastal Institute at URI (0504103), US NSF DEB Award (1049914), Philanthropic

Educational Organization (Lellis-Dib3158688), Northeast Aquatic Plant Management

Society, Rhode Island Natural History Survey and The Nature Conservancy of Rhode

Island (Lellis-Dibble 05-30-09), URI Agricultural Experiment Station (RI00H-332,

311000-6044), URI Coastal Fellows Program, and the U.S. and Czech Fulbright

Commissions.

\section{References}

Able, K.W., Hagan, S.M. \& Brown, S.A. (2003) Mechanisms of marsh habitat alteration due to Phragmites: response of young-of-the-year mummichog (Fundulus heteroclitus) to treatment for Phragmites removal. Estuaries, 26(2B), 484-494.

Beck, M.W., Heck, K.L. Jr., Able, K.W., Childers, D.L., Eggleston, D.B., Gillanders, B.M., Halpern, B., Hays, C.G., Hoshino, K., Minello, T.J., Orth, R.J., Sheridan, P.F. \& Weinstein, M.P. (2001) The identification, conservation, and management of estuarine and marine nurseries for fish and invertebrates. Bioscience, 52(8), 633-641.

Bond, A.L. \& Diamond, A.W. (2011) Recent Bayesian stable-isotope mixing models are highly sensitive to variation in discrimination factors. Ecological Applications, 21(4), 1017-1023.

Brittain, R.A., Schimmelmann, A., Parkhurst, D.F. \& Craft, C.B. (2012) Habitat use by coastal birds inferred from stable carbon and nitrogen isotopes. Estuaries and Coasts, 35, 633-645.

Bushaw-Newton, K.L., Kreeger, D.A., Doaty, S. \& Velinsky, D.J. (2008) Utilization of Spartina- and Phragmites-derived dissolved organic matter by bacteria and ribbed mussels (Geukensia demissa) from Delaware Bay salt marshes. Estuaries and Coasts, 31, 694-703.

Callaway, R.M. \& Ridenour, W.M. (2004) Novel weapons: invasive success and the evolution of increased competitive ability. Frontiers in Ecology and the Environment, 2(8), 436-443. 
Caut, S., Angulo, E. \& Courchamp, F. (2009) Variation in discrimination factors $\left(\Delta^{15} \mathrm{~N}\right.$ and $\left.\Delta{ }^{13} \mathrm{C}\right)$ : the effect of diet isotopic values and applications for diet reconstruction. Journal of Applied Ecology, 46, 443-453.

Chambers, R.M., Meyerson, L.A. \& Saltonstall, K. (1999) Expansion of Phragmites australis into tidal wetlands of North America. Aquatic Botany, 64, 261-273.

Chanton,J.P. \& Lewis, F.G. (1999) Plankton and dissolved inorganic carbon isotopic composition in a river-dominated estuary: Apalachicola Bay, Florida. Estuaries, 22(3A), 575-583.

Childers, D.L., Day, J.W. Jr. \& McKellar, H.N. Jr. (2000) Twenty more years of marsh and estuarine flux studies: revisiting Nixon (1980). Concepts and controversies in tidal marsh ecology (eds M.P. Weinstein \& D.A. Kreeger), pp. 391-424). Kluwer Academic Publishers, Netherlands.

Cloern, J.E., Canuel, E.A. \& Harris, D. (2002) Stable carbon and nitrogen isotope composition of aquatic and terrestrial plants of the San Francisco Bay estuarine system. Limnology and Oceanography, 7(3), 713-729.

Couch, C.A. (1989) Carbon and nitrogen stable isotopes of meiobenthos and their food resources. Estuarine Coastal and Shelf Science, 28, 433-441.

Currin, C.A., Newell, S.Y. \& Paerl, H.W. (1995) The role of standing dead Spartina alterniflora and benthic microalgae in salt marsh food webs: considerations based on multiple stable isotope analysis. Marine Ecology Progress Series, 121, 99-116.

Currin, C.A., Wainright, S.C., Able, K.W., Weinstein, M.P. \& Fuller, C.M. (2003) Determination of food web support and trophic position of the mummichog, Fundulus heteroclitus, in New Jersey smooth cordgrass (Spartina alterniflora), common reed (Phragmites australis), and restored salt marshes. Estuaries, 26, 495-510.

Darnell, R.M. (1967) Organic detritus in relation to the estuarine ecosystem. Estuaries (ed G.H. Lauff), pp. 376-382. AAAS Publication 83, Washington, D.C.

Deegan, L.A., Bowen, J.L., Drake, D., Fleeger, J.W., Friedrichs, C.T., Galván, K.A., Hobbie, J.E., Hopkinson, C., Johnson, D.S., Johnson, J.M., LeMay, L.E., Miller, E., Peterson, B.J., Picard, C., Sheldon, S., Sutherland, M., Vallino, J. \& Warren, R.S. (2007) Susceptibility of salt marshes to nutrient enrichment and predator removal. Ecological Applications, 17(5), S42-S63.

Deegan, L.A. \& Garritt, R.H. (1997) Evidence for spatial variability in estuarine food webs. Marine Ecology Progress Series, 147, 31-47. 
Deegan, L.A., Hughes, J.E. \& Rountree, R.A. (2000) Salt marsh ecosystem support of marine transient species. Concepts and controversies in tidal marsh ecology (eds M.P. Weinstein \& D.A. Kreeger), pp. 333-368. Kluwer Academic Publishers, Netherlands.

Dennis, C.A., MacNeil, M.A., Rosati, J.Y., Pitcher, T.E. \& Fisk, A.T. (2010) Diet discrimination factors are inversely related to $\delta^{15} \mathrm{~N}$ and $\delta^{13} \mathrm{C}$ values of food for fish under controlled conditions. Rapid Communications in Mass Spectrometry, 24, 3515-3520.

Dobush, G.R., Ankney, C.D. \& Krementz, D.G. (1985) The effect of apparatus, extraction time, and solvent type on lipid extractions of snow geese. Canadian Journal of Zoology, 63, 1917-1920.

Doucette, J.L., Wissel, B. \& Somers, C.M. (2011) Cormorant-fisheries conflicts: stable isotopes reveal a consistent niche for avian piscivores in diverse food webs. Ecological Applications, 21(8), 2987-3001.

Elsdon, T.S., Ayvazian, S., McMahon, K.W. \& Thorrold, S.R. (2010) Experimental evaluation of stable isotope fractionation in fish muscle and otoliths. Marine Ecology Progress Series, 408, 195-205.

Fry, B. (1988) Food web structure on Georges Bank from stable C, N, and S isotopic compositions. Limnology and Oceanography, 33(5), 1182-1190.

Fry, B. (2002) Conservative mixing of stable isotopes across estuarine salinity gradients: a conceptual framework for monitoring watershed influences on downstream fisheries production. Estuaries, 25(2), 264-271.

Fry, B., Cieri, M., Hughes, J., Tobias, C., Deegan, L.A. \& Peterson, B. (2008) Stable isotope monitoring of benthic-planktonic coupling using salt marsh fish. Marine Ecology Progress Series, 369, 193-204.

Fry, B. \& Sherr, E.B. (1984) Delta 13C measurements as indicators of carbon flow in marine and freshwater ecosystems. Contributions in Marine Science, 27, 1347.

Galván, K., Fleeger, J.W. \& Fry, B. (2008) Stable isotope addition reveals dietary importance of phytoplankton and microphytobenthos to saltmarsh infauna. Marine Ecology Progress Series, 359, 37-49.

Galván, K., Fleeger, J.W., Peterson, B., Drake, D., Deegan, L.A. \& Johnson, D.S. (2011) Natural abundance stable isotopes and dual isotope tracer additions help to resolve resources supporting a saltmarsh food web. Journal of Experimental Marine Biology and Ecology, 410, 1-11. 
Gratton, C. \& Denno, R.F. (2005) Restoration of arthropod assemblages in a Spartina salt marsh following removal of the invasive plant Phragmites australis. Restoration Ecology, 13(2), 358-372.

Gratton, C. \& Denno, R.F. (2006) Arthropod food web restoration following removal of an invasive wetland plant. Ecological Applications, 16, 622-631.

Grosholz, E. (2010) Avoidance by grazers facilitates spread of an invasive hybrid plant. Ecology Letters, 13, 145-153.

Hendricks, L.G., Mossop, H.E. \& Kicklighter, C.E. (2011) Palatability and chemical defense of Phragmites australis to the marsh periwinkle snail Littoraria irrorata. Journal of Chemical Ecology, 37, 838-845.

Hughes, E.H. \& E.B. Sherr (1983) Subtidal food webs in a Georgia estuary: $\delta^{13} \mathrm{C}$ analysis. Journal of Experimental Marine Biology and Ecology, 67, 227-242.

Jackson, A.L., Inger, R., Bearhop, S. \& Parnell, A. (2009) Erroneous behavior of MixSIR, a recently published Bayesian isotope mixing model: a discussion of Moore \& Semmens (2008). Ecology Letters, 12, E1—E5.

Jacob, U., Mintenbeck, K., Brey, T., Knust, R. \& Beyer, K. (2005) Stable isotope food web studies: a case for standardized sample treatment. Marine Ecology Progress Series, 287, 251-253.

Jiménez, J.M., Wieski, K., Marczak, L.B., Ho, C.K. \& Pennings, S.C. (2012) Effects of an omnivorous katydid, salinity, and nutrients on a planthopper-Spartina food web. Estuaries and Coasts, 35, 475-485.

Kneib, R.T., Stiven, A.E. \& Haines, E.B. (1980) Stable carbon isotope ratios in Fundulus heteroclitus (L.) muscle tissue and gut contents from a North Carolina Spartina marsh. Journal of Experimental Marine Biology and Ecology, 46, 89-98.

Litvin, S.Y. \&Weinstein, M.P. (2003) Life history strategies of estuarine nekton: the role of marsh macrophytes, benthic microalgae, and phytoplankton in the trophic spectrum. Estuaries, 26(2B), 552-562.

Litvin, S.Y. \&Weinstein, M.P. (2004) Multivariate analysis of stable-isotope ratios to infer movements and utilization of estuarine organic matter by juvenile weakfish (Cynoscion regalis). Canadian Journal of Fisheries and Aquatic Sciences, 61, 1851-1861.

Martínez del Rio, C., Wolf, N., Carleton, S.A. \& Gannes, L.Z. (2009) Isotopic ecology ten years after a call for more laboratory experiments. Biological Reviews, 84, 91-111. 
McCutchan, J.H. Jr., Lewis, W.M. Jr., Kendall, C. \& McCrath, C.C. (2003) Variation in trophic shift for stable isotope ratios of carbon, nitrogen, and sulfur. OIKOS, 102, 378-390.

Meyerson, L.A., Saltonstall, K. \& Chambers, R.M. (2009) Phragmites australis in Eastern North America: A historical and ecological perspective. Salt Marshes Under Global Siege (B.R. Silliman, E. Grosholz, \& M.D. Bertness), pp. 57-82. University of California Press.

Meyerson, L.A., Saltonstall, K., Windham, L., Kiviat, E. \& Findlay, S. (2000) A comparison of Phragmites australis in freshwater and brackish marsh environments in North America. Wetlands Ecology and Management, 8, 89103.

Nixon, S.W. (1980) Between coastal marshes and coastal waters: a review of twenty years of speculation and research on the role of salt marshes in estuarine productivity and water chemistry. Estuarine and Wetland Processes (eds P. Hamilton \& K.B. MacDonald), pp. 437-525. Plenum Press, New York.

O’Brien, D.M., Schrag, D.P. \& Martínez del Rio, C. (2000) Allocation to reproduction in a hawkmoth: a quantitative analysis using stable carbon isotopes. Ecology, 81(10), 2822-2831.

Odum, E.P. (1968) A research challenge: evaluating the productivity of coastal and estuarine water. Proceedings, 2nd Sea Grant Conference, Graduate School of Oceanography. University of Rhode Island, Kingston, Rhode Island.

Orr, S.P., Rudgers, J.A. \& Clay, K. (2005) Invasive plants can inhibit native tree seedlings- testing potential allelopathic mechanisms. Plant Ecology, 181, 153165.

Parker, J.D., Montoya, J.P. \& Hay, M.E. (2008) A specialist detritivore links Spartina alterniflora to salt marsh food webs. Marine Ecology Progress Series, 364, 8795.

Parnell, A.C., Inger, R., Bearhop, S. \& Jackson, A.L. (2010) Source partitioning using stable isotopes: coping with too much variation. PLoS ONE, 5(3), e9672. doi:10.1371/journal.pone.0009672.

Pennings, S.C., Carefoot, T.H., Siska, E.L., Chase, M.E. \& Page, T.A. (1998) Feeding preferences of a generalist salt marsh crab: relative importance of multiple plant traits. Ecology, 79(6), 1968-1979.

Peterson, B.J. \& Fry, B. (1987) Stable isotopes in ecosystem studies. Annual Review of Ecology and Systematics, 18, 293-320. 
Pinnegar, J.K. \& Polunin, N.V.C. (1999) Differential fractionation of $\delta^{13} \mathrm{C}$ and $\delta^{15} \mathrm{~N}$ among fish tissues: implications for the study of trophic interactions. Functional Ecology, 13, 225-231.

Post, D.M. (2002) Using stable isotopes to estimate trophic position: models, methods, and assumptions. Ecology, 83(3), 703-718.

Post, D.M., Layman, C.A., Arrington, D.A., Takimoto, G., Quattrochi, J. \& Montana, C.G. (2007) Getting to the fat of the matter: models, methods and assumptions for dealing with lipids in stable isotope analyses. Oecologia, 152, 179-189.

Redfield, A.C. (1958) The biological control of chemical factors in the environment. American Scientist, 46(3), 230A, 205-221.

Rooth, J.E., Court Stevenson, J. \& Cornwell, J.C. (2003) Increased sediment accretion rates following invasion by Phragmites australis: the role of litter. Estuaries, 26(2B), 475-483.

Teal, J.M. (1962) Energy flow in the salt marsh ecosystem of Georgia. Ecology, 43, 614-24.

Vanderklift, M.A. \& Ponsard, S. (2003) Sources of variation in consumer-diet $\delta^{15} \mathrm{~N}$ enrichment: a meta-analysis. Oecologia, 136, 169-182.

Wainright, S.C., Weinstein, M.P., Able, K.W. \& Currin, C.A. (2000) Relative importance of benthic microalgae, phytoplankton, and the detritus of smooth cordgrass Spartina alterniflora and the common reed Phragmites australis to brackish-marsh food webs. Marine Ecology Progress Series, 200, 77-91.

Weinstein, M.P., Litvin, S.Y., Bosley, K.L., Fuller, C.M. \& Wainright, S.C. (2000) The role of tidal marsh as an energy source for marine transient and resident finfishes: a stable isotope approach. Transactions of the American Fisheries Society, 129, 797-810.

Weinstein, M.P., Litvin, S.Y. \& Guida, V.G. (2009) Essential Fish Habitat and wetland restoration success: a tier III approach to the biochemical condition of common mummichog Fundulus heteroclitus in common reed Phragmites australis and smooth cordgrass Spartina alterniflora-dominated salt marshes. Estuaries and Coasts, 32, 1011-1022.

Weis, J.S. (2005) Diet and food web support of the white perch, Morone americana, in the Hackensack Meadowlands of New Jersey. Environmental Biology of Fishes, 74, 109-113. 
Wozniak, A.S., Roman, C.T., Wainright, S.C., McKinney, R.A. \& James-Pirri, M.J. (2006) Monitoring food web changes in tide-restored salt marshes: a carbon stable isotope approach. Estuaries and Coasts, 29(4), 568-578.

Wyatt, A.S.J., Waite, A.M. \& Humphries, S. (2010) Variability in isotope discrimination factors in coral reef fishes: implications for diet and food web reconstruction. PLoS ONE, 5(10), e13682. doi:10.1371/journal.pone.0013682.

Zedler, J.B. (1980) Algal mat productivity: comparisons in a salt marsh. Estuaries, 3(2), 122-131. 
TABLES: CHAPTER 2

Table 2-1. Summary of data for primary producers, fish, and eggs by marsh type.*

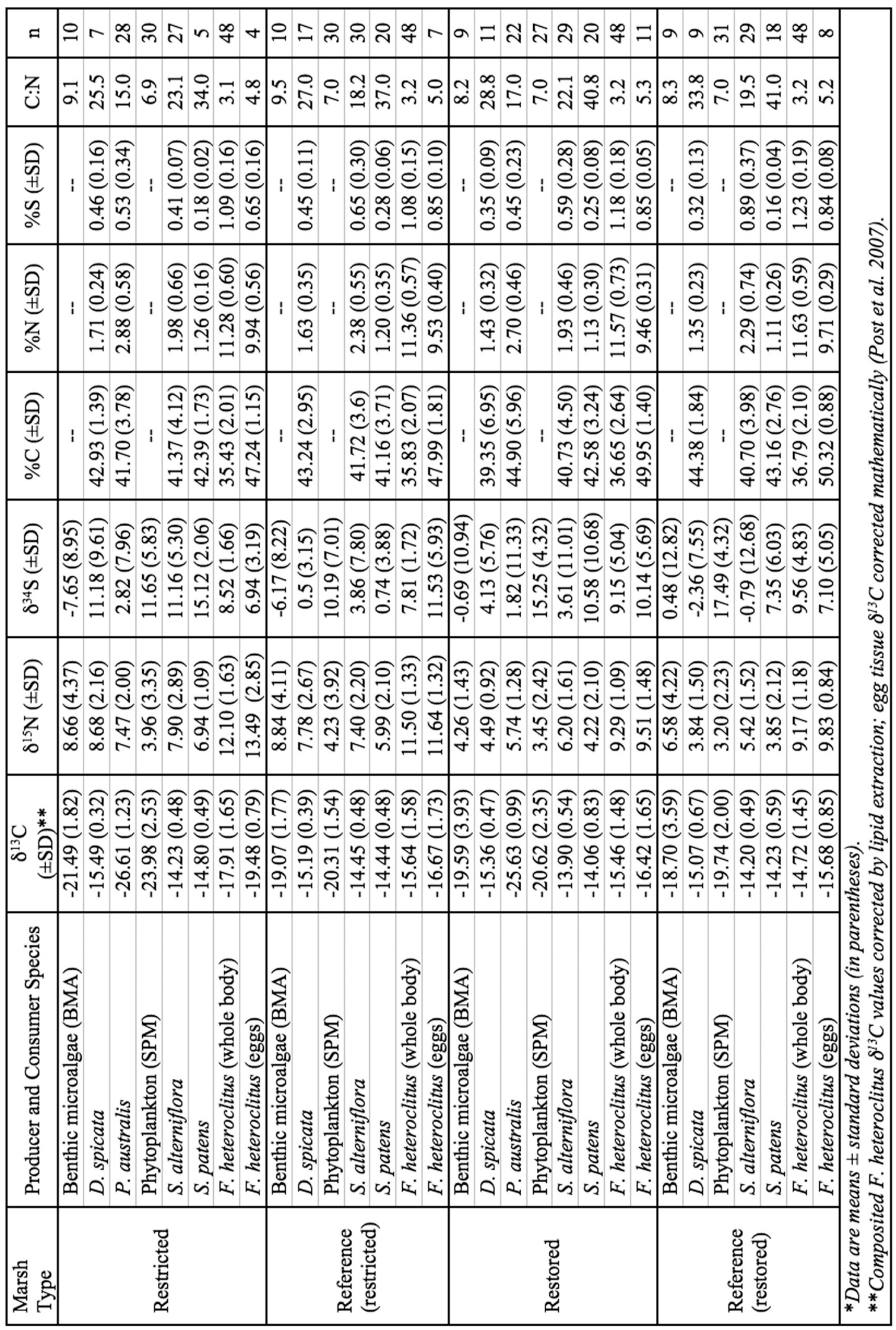


Table 2-2. Discrimination factors used in sensitivity analysis with model output.

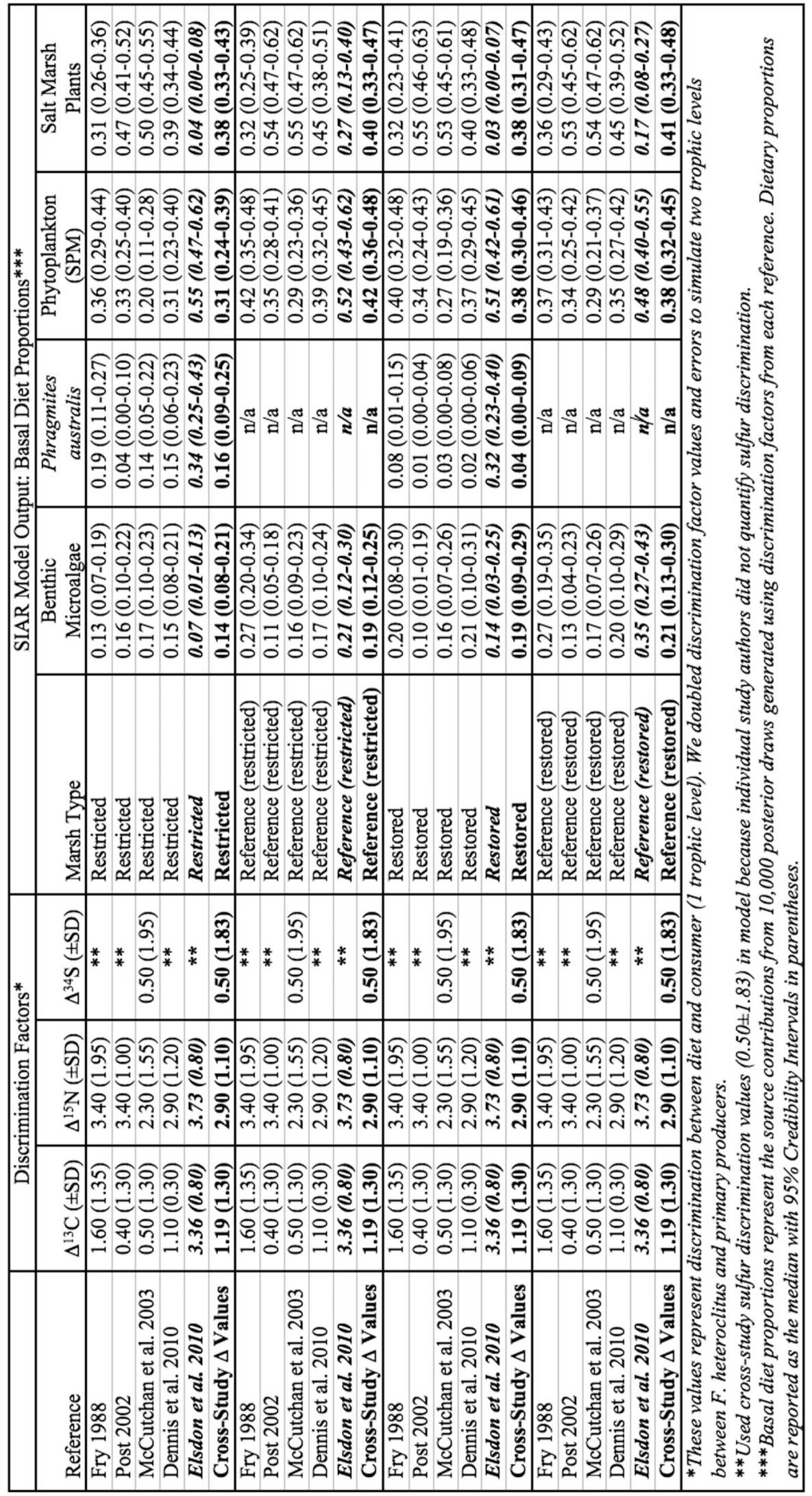




\section{FIGURES: CHAPTER 2}

Figure 2-1. Map of study site locations.

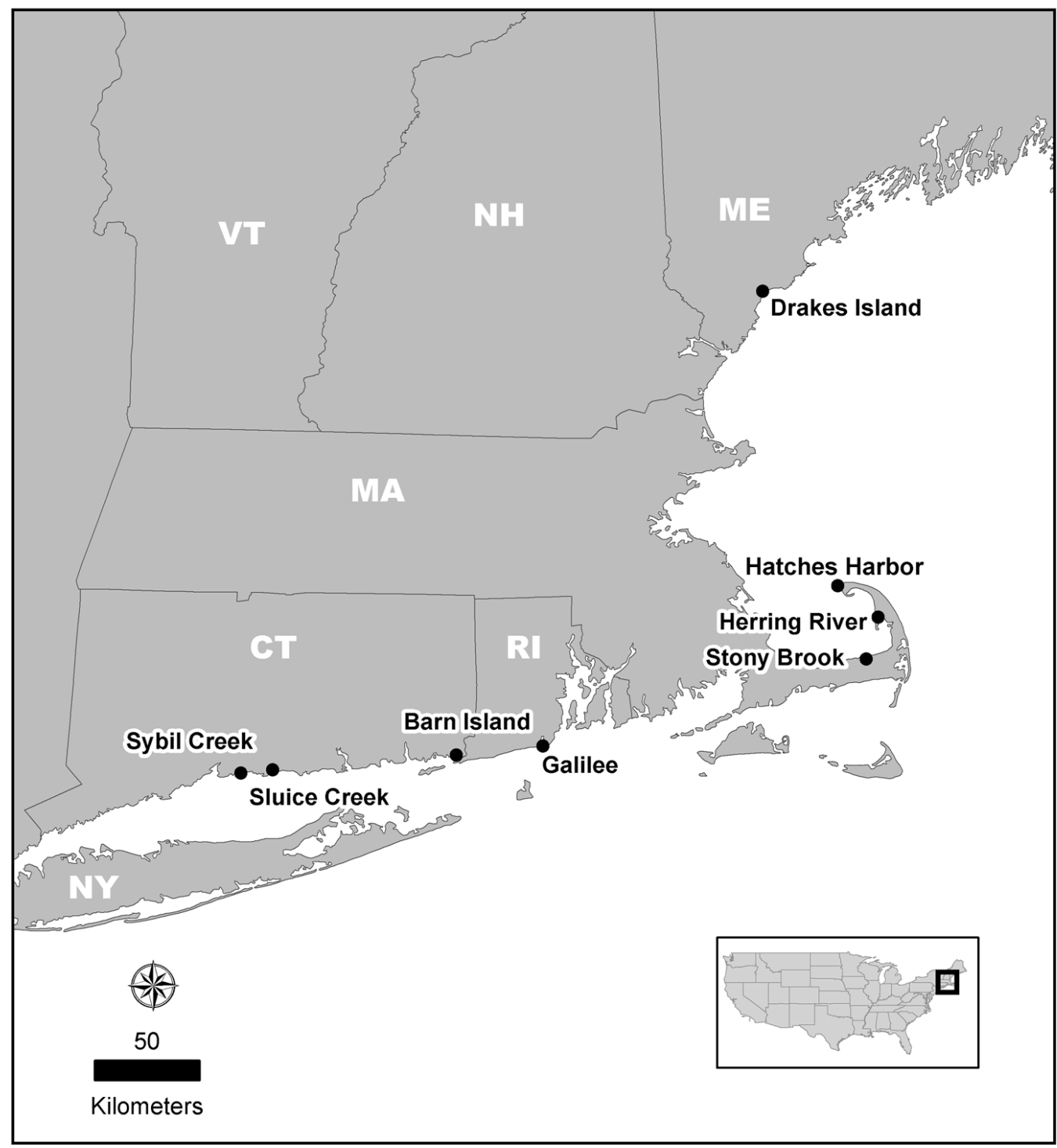


Figure 2-2. Carbon stable isotope composition of suspended particulate matter (SPM) and dissolved inorganic carbon (DI) vs. water column salinity for all study sites.

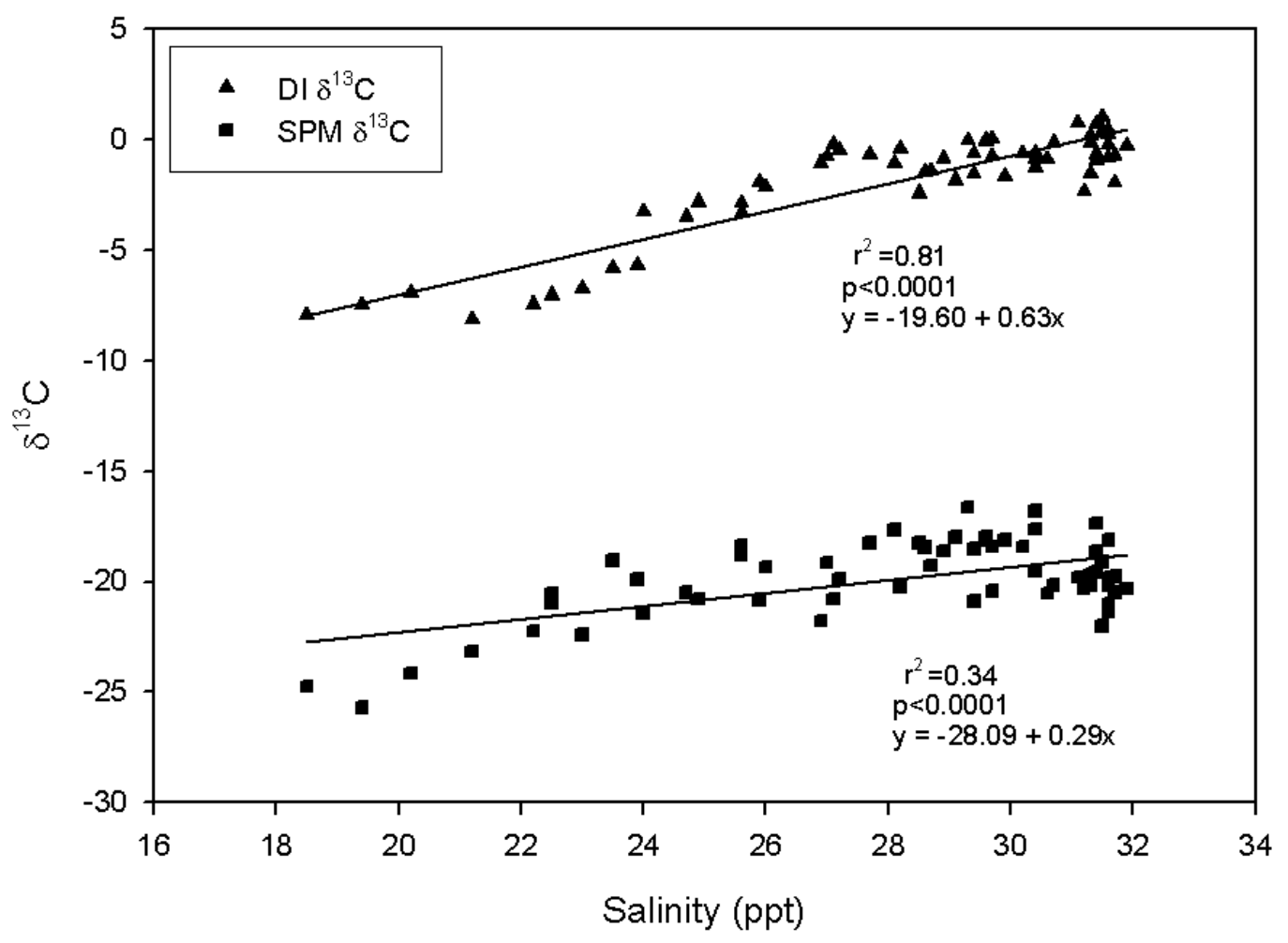


Figure 2-3. Relationship between the carbon isotopic compositions of suspended particulate matter (SPM) and dissolved inorganic carbon (DI) in the water column for all study sites.

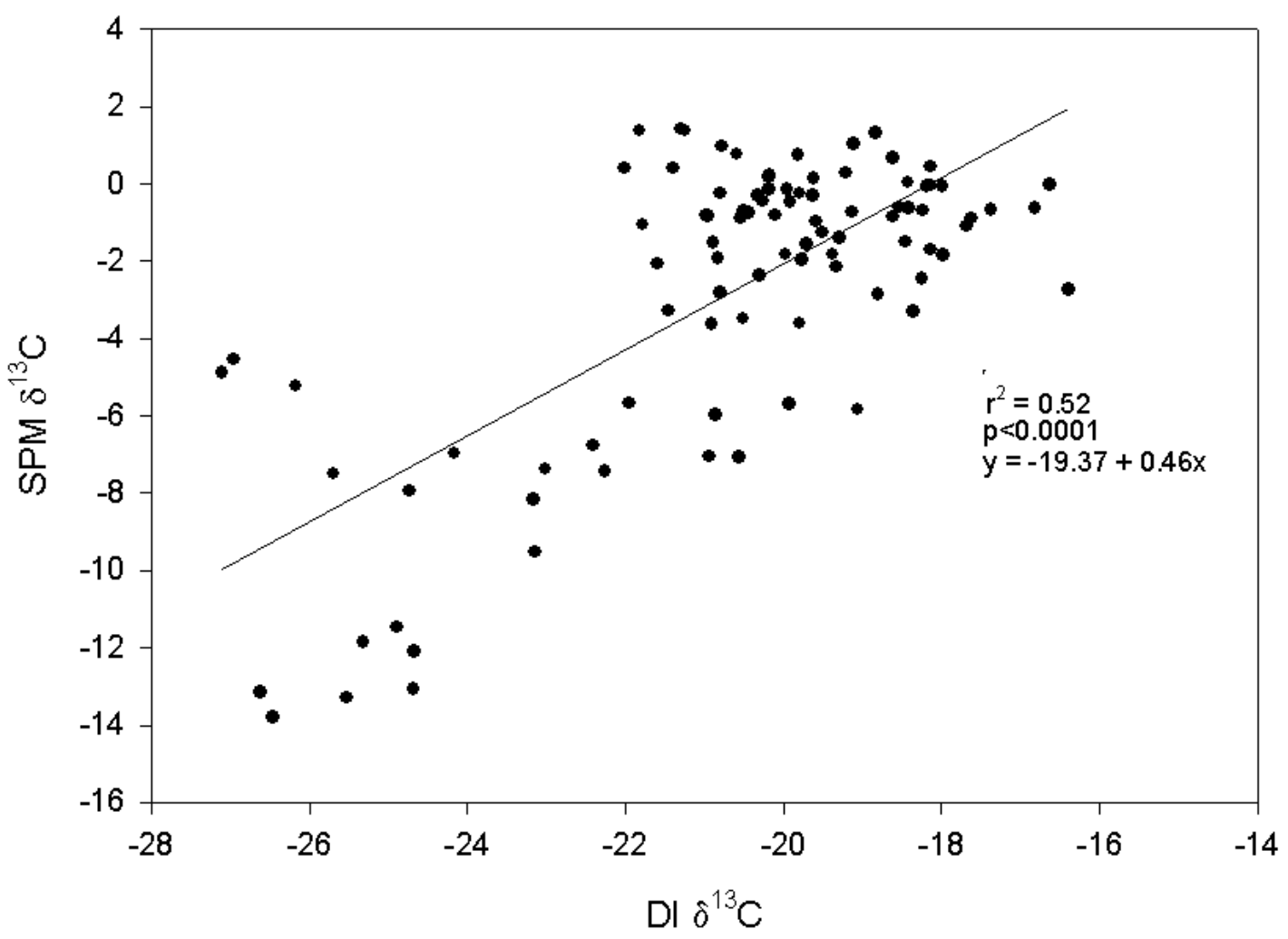


Figure 2-4. Stable isotope biplots for (a) $\delta^{13} \mathrm{C}$ vs. $\delta^{15} \mathrm{~N}$ and (b) $\delta^{13} \mathrm{C}$ vs. $\delta^{34} \mathrm{~S}$ for the restricted vs. reference (control) marsh sites. Data for sources are means \pm standard deviations and are separated by marsh type. Key: Fish $=F$. heteroclitus (whole body, lipid-free); $\mathrm{BMA}=$ benthic microalgae; $\mathrm{SPM}=$ phytoplankton; $\mathrm{SPDI}=$ salt marsh plants; PHAU $=$ Phragmites australis. Fish data is not corrected for discrimination in these plots.

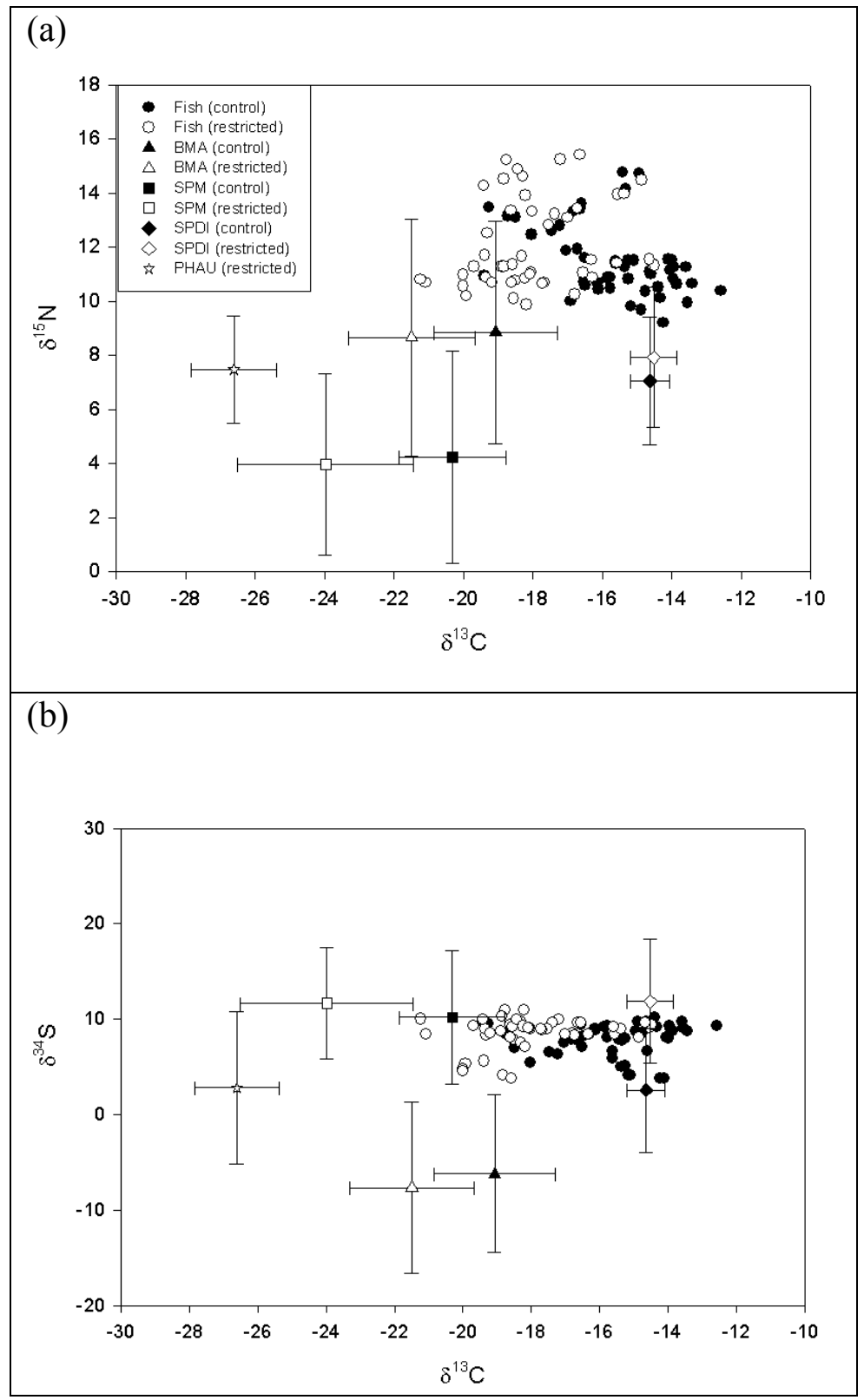


Figure 2-5. Stable isotope biplots for (a) $\delta^{13} \mathrm{C}$ vs. $\delta^{15} \mathrm{~N}$ and (b) $\delta^{13} \mathrm{C}$ vs. $\delta^{34} \mathrm{~S}$ for the restored vs. reference (control) marsh sites. Data for sources are means \pm standard deviations and are separated by marsh type. Key: Fish $=F$. heteroclitus (whole body, lipid-free); $\mathrm{BMA}=$ benthic microalgae; $\mathrm{SPM}=$ phytoplankton; $\mathrm{SPDI}=$ salt marsh plants; PHAU $=$ Phragmites australis. Fish data is not corrected for discrimination in these plots.

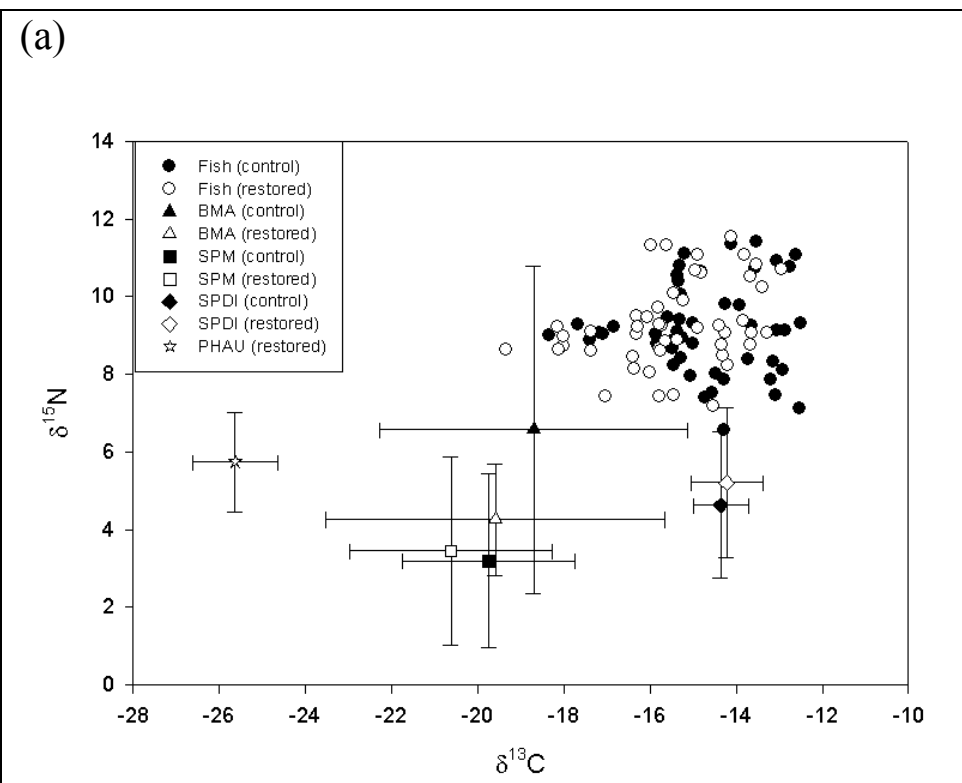

(b)

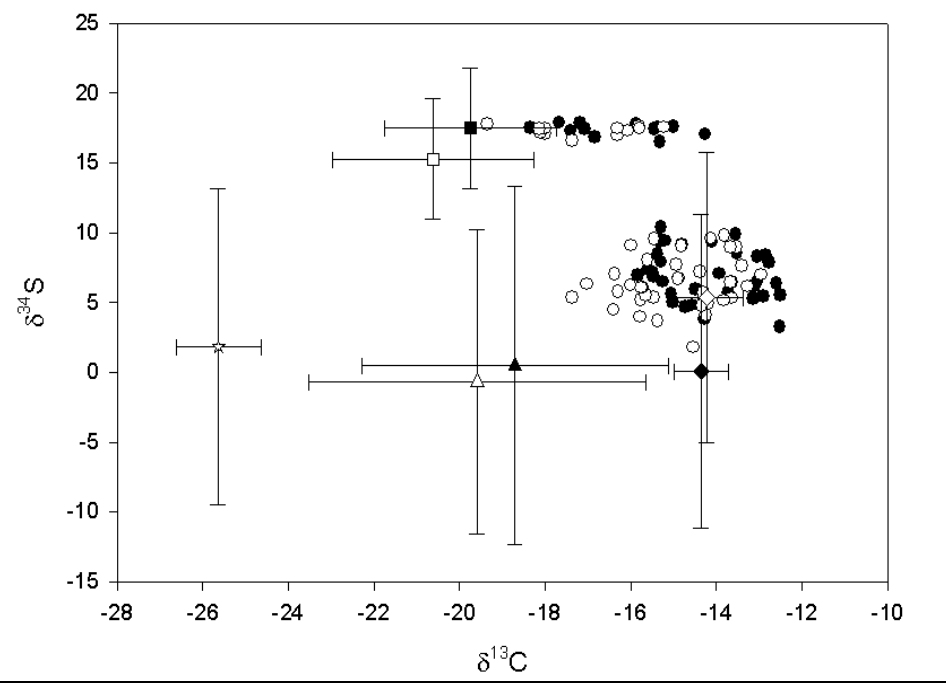




\section{CHAPTER 3}

Tidal flushing restores the physiological condition of fish residing in degraded salt marshes

by

Kimberly L. Dibble ${ }^{1}$; Laura A. Meyerson ${ }^{1,2}$

was published in PLoS ONE (September 2012)

${ }^{1}$ Department of Natural Resources Science, University of Rhode Island, 1 Greenhouse Road, Kingston, RI 02881.

${ }^{2}$ Institute of Botany, Department of Invasion Ecology, Academy of Sciences of the Czech Republic, Průhonice, CZ 252 43, Czech Republic. 


\begin{abstract}
Roads, bridges, and dikes constructed across salt marshes can restrict tidal flow, degrade habitat quality for nekton, and facilitate invasion by non-native plants including Phragmites australis. Introduced $P$. australis contributes to marsh accretion and eliminates marsh surface pools thereby adversely affecting fish by reducing access to intertidal habitats essential for feeding, reproduction, and refuge. Our study assessed the condition of resident fish populations (Fundulus heteroclitus) at four tidally restricted and four tidally restored marshes in New England invaded by $P$. australis relative to adjacent reference salt marshes. We used physiological and morphological indicators of fish condition, including proximate body composition (\% lipid, \% lean dry, \% water), recent daily growth rate, age class distributions, parasite prevalence, female gravidity status, length-weight regressions, and a common morphological indicator (Fulton's K) to assess impacts to fish health. We detected a significant increase in the quantity of parasites infecting fish in tidally restricted marshes but not in those where tidal flow was restored to reduce $P$. australis cover. Using fish length as a covariate, we found that unparasitized, non-gravid $F$. heteroclitus in tidally restricted marshes had significantly reduced lipid reserves and increased lean dry (structural) mass relative to fish residing in reference marshes. Fish in tidally restored marshes were equivalent across all metrics relative to those in reference marshes indicating that habitat quality was restored via increased tidal flushing. Reference marshes adjacent to tidally restored sites contained the highest abundance of young fish (ages 0-1) while tidally restricted marshes contained the lowest. Results indicate that $F$. heteroclitus residing in physically and hydrologically
\end{abstract}


altered marshes are at a disadvantage relative to fish in reference marshes but the effects can be reversed through ecological restoration.

Keywords: Fundulus heteroclitus; Phragmites australis; invasion; lipid; otolith; age; growth rate; length-weight; parasite; gravid; nursery; fish habitat

\section{Introduction}

It is well established that fish and swimming crustaceans (termed "nekton") use vegetated intertidal salt marsh habitats for refuge, feeding, as nurseries, and for reproduction [1-6]. Although there has been a long-standing debate on the role of salt marsh detritus in the direct support of higher trophic levels [7-11], several studies have linked access to invertebrate prey on the marsh surface to measurable changes in fish growth, weight gain, and energy storage [4,12-16]. High quality salt marsh habitat facilitates secondary production in coastal waters as nekton are consumed by higher trophic levels [17-19].

Throughout the United States, $>50 \%$ of tidal salt marshes have decreased in size and quality [20] because of disturbances such as interstate commerce, urban and shoreline development, and livestock rearing [21,22]. Roads, bridges, and dikes constructed through salt marshes restrict tidal flow when associated culverts are undersized, resulting in marsh compaction and subsidence through the loss of inorganic sediments from tidal deposition and the oxidation and decay of drained peat deposits [23]. Tidal 
restrictions also facilitate plant invasions and further degrade habitat quality for resident nekton species $[24,25]$.

Introduced Phragmites australis subsp. australis (hereafter, "introduced P. australis") has widely invaded oligohaline to polyhaline salt marshes throughout the mid-Atlantic and New England regions of North America [26-28]. This invasive macrophyte takes advantage of reduced salinity and increased disturbance and nitrogen availability behind tidal restrictions and forms near monocultures that decrease native plant diversity, temperature, and light $[29,30]$. The dense belowground network of introduced $P$. australis roots and rhizomes and high aboveground biomass mat of living and slowly decomposing organic matter [29] that traps mineral and organic sediment can counteract the effects of marsh subsidence by raising marsh surface elevation. However, high rates of marsh accretion (3-4mm per year) [31] can elevate the marsh platform to the extent that daily high tides may no longer flood the marsh surface [22]. In addition, during the later stages of $P$. australis invasion small waterfilled marsh pools and depressions are often reduced [32,33]. Restoration of tidal flow into restricted marshes has successfully decreased the cover of this invader $[24,30,34,35]$ and restored ecological function for multiple taxa $[25,36,37]$.

Previous studies in New England have used measures of faunal presence/absence, quantity, richness, and diversity to assess habitat quality in tidally restricted marshes invaded by $P$. australis and tidally restored marshes relative to reference (Spartina alterniflora) marshes. Decreases in bird species richness, density, and abundance were 
documented in restricted marshes [36,38], but nekton response was variable across studies, with density, abundance, and species richness varying by site and species $[25,36,37,39,40]$. Tidally restored sites exhibit wide variation in support of nekton for several years post-restoration while hydrologic, environmental, and physical variables respond over time to increased tidal flooding and duration [25,36,41,42]. Raposa and Talley [43] suggest the variability in restoration response may be related to whether the marsh was previously diked/drained or diked/impounded, with the former showing increased nekton density and the latter showing decreased nekton density postrestoration.

Several studies have acknowledged the need to move beyond the collection of community data (e.g., density, richness) to assess the functional response of nekton to tidal restrictions and restoration $[16,43,44]$. Fish condition and growth are affected by habitat characteristics (e.g., prey availability, predation, competition, water quality, parasite presence, etc.) and by the physiology of the fish species (e.g., reproductive status, life history stage, sex, etc.) [45-48]. Fish exhibit life-long tradeoffs in resource allocation to metabolism, somatic growth, reproduction, and energy (lipid) storage $[49,50]$, with the latter essential to their ability to cope with environmental stress and successfully overwinter in northern climates [49,51]. Resident salt marsh fish such as the mummichog, Fundulus heteroclitus, gain a significant portion of their energy by foraging on the marsh surface at high tide but show significant decreases in growth rate and weight gain when they only have access to unvegetated creek beds and pools $[4,12,14]$. Therefore, a decrease in marsh surface access or habitat quality resulting 
from tidal restrictions and $P$. australis invasion may result in detectable tradeoffs to fish condition, growth, and ultimately, survival.

Morphological and physiological indicators have been used to examine habitat quality for fish residing in different environments $[5,16,46,48,52-54]$. At the morphological level, the relationship between fish length and wet weight using regression and indices such as the Fulton's Condition Factor (K) can be used to infer the well being of fish and are based on the premise that heavier fish of a given length are in better condition [55]. At the biochemical level the analysis of proximate body composition (\% lipid, \% lean dry mass, \% water) is used to estimate resource allocation to energy storage vs. body structure $[49,52]$. Habitat quality influences fish growth rate; therefore, if a linear relationship exists between fish size and otolith size [56], the mean daily width of the marginal otolith increments can be used as an index of recent daily growth $[48,57,58]$. Further, age class distributions using the annuli of otoliths and scales provide information on habitat suitability for different life history stages $[54,59]$. Parasite prevalence and infection intensity have been used as indicators of environmental quality; however, the responses of parasite communities and their hosts vary depending on exposure time, parasite life cycle (direct or indirect), and environmental perturbations present (e.g., sewage, eutrophication, pollution, thermal stress, etc.) [53,60-62]. Nonetheless, parasites are energetically costly and infection may result in tradeoffs to lipid storage, reproduction, and growth $[16,61]$. 
Our study builds on earlier work by directly linking habitat quality to measurable attributes of fish health and productivity. We examined the influence of habitat quality on fish condition and growth using the above morphological and physiological indicators in order to address the following research questions: 1) Does the condition and growth of fish residing in tidally restricted marshes invaded by $P$. australis differ from fish in unrestricted, uninvaded (reference) marshes? 2) Can we detect a difference in the condition and growth of fish residing in reference marshes vs. those that have been tidally restored to remove $P$. australis? 3) Are differences in fish condition and growth between the restricted, restored, and reference marshes consistent across regions, seasons, and for both males and females?

\section{Methods}

\section{Ethics Statements}

Our study was carried out in strict accordance with the American Veterinary Medical Association Guidelines on Euthanasia and was approved by the University of Rhode

Island Institutional Animal Care and Use Committee (protocol \#AN09-05-020).

Permission for collections were given by the Connecticut Department of

Environmental Protection (\#SC-10021), Rhode Island Department of Environmental Management (\#2010-39), Massachusetts Division of Marine Fisheries (\#159948), National Park Service Cape Cod National Seashore (\#CACO-2010-SCI-0016), Rachel

Carson National Wildlife Refuge (\#53553-2009-05, 2010-05, 2011-10), and Maine

Department of Marine Resources (\#2009-53-00, 2010-60-01, 2011-45-02). 


\section{Study Sites and Sampling Locations}

We selected four tidally restricted (hereafter, "restricted") and four tidally restored ("restored") salt marshes invaded by introduced $P$. australis in New England spanning Connecticut to Maine (Fig. 3-1, Table 3-1). Each restricted or restored site was paired with an adjacent downstream, unrestricted ("reference") site that was sampled on the same day ( $\mathrm{n}=16$ marshes; 4 restricted, 4 restored, 8 reference). Three sampling stations were randomly selected a priori along the main tidal creek within each of the 16 marshes ( $\mathrm{n}=48$ experimental units). Because we employed a matched pairs experimental design, data from restricted marshes were only compared to data from the adjacent reference marshes, and data from restored marshes were only compared to data from adjacent reference marshes (i.e., four "marsh types" were analyzed; restricted/reference; restored/reference; Table 3-1). At the Stony Brook, MA site two undersized, failing culverts were replaced between the first and second year of our study due to funds appropriated for 'shovel-ready' habitat restoration projects (American Recovery and Reinvestment Act of 2009). However, because the other sites had tidal restrictions removed 11-22 years earlier we still classified year 2 data as restricted in the analysis.

Site characteristics are reviewed in Table 3-1 [63-76]. Introduced $P$. australis was more prevalent in restricted marshes than in the restored marshes (K.L. Dibble, personal observation). At restored marshes the increase in tidal flow and associated salinity over time has decreased the cover of introduced $P$. australis and/or forced distributional shifts of the invasive plant toward the upland edge of the marsh 
$[35,71,75]$. The restored marshes are all marsh meadow systems with restrictions dating back to 1848 that were put in place to enhance hunting, agriculture, commerce, and flood control. They have been undergoing restoration for 1-2 decades as evidenced by similarity in mean tidal range and salinity relative to adjacent reference marshes. The restricted marshes are all tidal riverine systems diked dating back to the 1700's for agriculture (salt hay farming), salt works, flood control, and/or to facilitate commerce/travel [63-76]. Mean tidal range and salinity in the restricted marshes is lower relative to adjacent reference marsh meadows and fringing marshes (Table 3-1), facilitating the observed invasion by $P$. australis. Although our study design does not allow us to separate the effects of tidal restrictions from the effects of $P$. australis invasion, these two factors are often successive in New England salt marshes and both work to reduce tidal range and marsh surface access and hence, nekton support functions $[24,40,71,77]$.

\section{Field Data}

We collected data on water column salinity (ppt), temperature $\left({ }^{\circ} \mathrm{C}\right)$, and dissolved oxygen (mg/L) at each station using a YSI-85 (2010) and a YSI Pro-2030 (2011). Water quality data were spot measurements ( $\mathrm{n}=1$ per station per time period) taken from approximately mid-way through flood tide to peak high tide (prior to ebbing) when fish were removed from the water column. We collected water quality data from all sites in fall 2010, summer 2011, and fall 2011, but only from the four southern sites in Connecticut and Rhode Island in summer 2010 (due to equipment malfunction). Sampling dates were as follows: summer 2010 (7/12-7/25, 7/29), fall $2010(9 / 22-$ 
10/3), summer $2011(7 / 11-7 / 23)$, and fall 2011 (9/25-10/7). Study sites were sampled along a south-to-north transect in summer, and then along a north-to-south transect in fall to account for seasonality changes in the marshes. For gravidity data, sites were sampled during one lunar cycle in summer 2010 (new moon on 7/11/10, full moon on 7/26/10), while sites were sampled during the days leading up to and just past full moon $(7 / 15 / 11)$ in summer 2011.

On flood tide at each station on every sample date we deployed two minnow traps containing bait in enclosed mesh packets (to prevent consumption). All traps were placed within one meter of the salt marsh bank parallel to the shore in the main tidal creek of each system [78]. After 30 minutes we combined the fish contents from both traps and randomly selected eight male and eight female adult $F$. heteroclitus $(>40 \mathrm{~mm}$ in fork length) representing the longest (2 male, 2 female), shortest (2 M, $2 \mathrm{~F}$ ), and intermediate (4 M, 4 F) size ranges of fish available. Sixteen fish were selected per station (15 fish analyzed, 1 stored in $-80^{\circ} \mathrm{C}$ freezer) because previous power analyses and other analyses using nekton species composition, abundance, length, and biochemical data indicated that replicate samples of 5-15 F. heteroclitus was sufficient to detect trends between marsh types $[16,79,80]$. We measured fork length (nearest millimeter) and wet weight (nearest centigram), quantified external parasites (ectoparasites) on the body surface, and then humanely euthanized fish in the field using a sharp knife and the guillotine method. In the laboratory, we quantified internal parasites (endoparasites) infecting the liver, heart, and abdominal cavity, recorded female gravidity status (eggs present/absent), and removed and discarded fish 
digestive tracts and regurgitated food. We calculated parasite infection intensity, prevalence, and weighted prevalence [81,82] using all data from 2010-2011. We rinsed fish in DI water then froze and freeze-dried the 16 fish from each station. Of the 16 fish, five female and five male fish were randomly selected, ground, and stored in a $-80^{\circ} \mathrm{C}$ freezer for lipid extraction. Five fish $(2$ male $/ 3$ females, or 3 female $/ 2$ males $)$ from each station were stored in a $-80^{\circ} \mathrm{C}$ freezer, with the field-decapitated head used for otolith measurements.

\section{Laboratory Data}

\section{Physiological Condition and Growth}

\section{Proximate Body Composition (Lipid/Lean Dry/Water)}

In 2010 and 2011 we extracted whole-body lipid reserves from 1,920 adult fish ( $\mathrm{n}=960$ fish/year). Powdered fish samples were packed into pre-weighed Whatman cellulose extraction thimbles, dried to a constant weight in a $50^{\circ} \mathrm{C}$ oven overnight, reweighed pre-extraction, extracted for six hours using petroleum ether and a Soxhlet apparatus, dried in a $50^{\circ} \mathrm{C}$ oven overnight, and then re-weighed post-extraction [83]. We selected petroleum ether as the non-polar lipid solvent because it is highly effective at removing neutral lipids (energy reserves) while minimizing loss of nonlipid, structural material [83]. We determined the percent lipid (\% dry), lean dry mass ( $\%$ dry), and water (\% wet) of individual fish using the following equations:

(i) $\%$ lipid $=[($ pre-extraction dry wt. (g) - post-extraction dry wt. (g))/(preextraction dry wt. (g) - dry thimble weight (g)] x 100 
(ii) $\%$ lean dry mass $=[($ post-extraction dry wt. $(\mathrm{g})-$ dry thimble wt. $(\mathrm{g})) /($ preextraction dry wt. (g) - dry thimble wt. (g)] x 100

(iii) $\%$ water $=[$ fish wet wt. in field $(\mathrm{g})-($ pre-extraction dry wt. $(\mathrm{g})-$ dry thimble wt. (g))/fish wet wt. in field (g)] x 100

\section{Fish Age and Recent Daily Growth Rate}

Radtke and Dean [84] verified daily increment formation using $F$. heteroclitus sagittae, finding that daily increments form regardless of growth rate, which is faster at higher water temperatures $\left(30^{\circ} \mathrm{C}\right.$ vs. $\left.24^{\circ} \mathrm{C}\right)$. Therefore, we can use sagittal otolith increments to determine the age and growth rates of $F$. heteroclitus living in different environmental conditions. We removed pairs of sagittal otoliths from 960 adult fish ( $\mathrm{n}=480$ fish/year) using a dissecting microscope and the 'crunch and crumble' extraction method [85]. Otoliths were cleaned in distilled water and $10 \%$ bleach, treated with $95 \%$ ethanol, and then dried in an oven $\left(1 \mathrm{~h}\right.$ at $\left.50^{\circ} \mathrm{C}\right)$. We mounted the pair of otoliths on standard microscope slides (sulcus side down), covered in Cargille immersion oil (Type FF, nonfluorescing). All measurements were done using the right otolith for consistency, unless the right was broken or could not be located during extraction. In that case, measurements were done on the left otolith. Using a Zeiss Stereo Microscope (Discovery, v12), high-powered objective (Plan Apo S 3,5x), and image analysis software (AxioVisionRel.4.8), we recorded fish age under transmitted light (\# dark annular rings, magnification 100x) [59]. 
To verify the relationship between otolith growth and somatic growth [56], we took three measurements of total otolith radius $(\mu \mathrm{m})$ and calculated the mean. We also took three measurements of total otolith height and length and calculated the mean for each otolith. Under reflected light and high magnification (560x), we measured the distance between the margin of the otolith in the postero-dorsal region $[86,87]$ back to the $10^{\text {th }}$ daily growth ring three times, took the mean of the three separate measurements, and divided the measurement by 10 days to compute the Recent Growth Index (in $\mu \mathrm{m}$ ) [48,57]. Recent daily growth measurements from readable otoliths were re-measured by a second reader 2-3 months later. We discarded any otoliths for which the first and second growth measurements were not within $10 \%$ of each other and report the mean of the first and second measurements [88-91].

\section{Morphological Condition}

During field collections, we recorded the fork length and wet weight of 1,487 fish in 2010 and 1,529 fish in 2011. We use a common morphometric index of fish condition, Fulton's Condition Factor (K), to compare the condition of adult fish. It is calculated using the following equation:

$$
\text { (iv) } \mathrm{K}=100 *\left(\mathrm{~W} / \mathrm{L}^{3}\right)
$$

Where $\mathrm{W}=$ weight of fish $(\mathrm{mg})$ and $\mathrm{L}=$ fork length of fish (mm) [55]. Fulton's $\mathrm{K}$ assumes that heavier fish of a given length are in better condition; therefore this index can be used as an indicator of energy storage. We compared the results of $\mathrm{K}$ to the results of Multiple Linear Regression using categorical variables for each marsh type (restricted, restored, reference). 


\section{Statistical Analyses}

In total, our main experiment included two paired marsh comparisons (restricted vs. reference; restored vs. reference). Each of the 48 Experimental Units (EU) were visited twice in $2010(\mathrm{n}=96)$ and twice in $2011(\mathrm{n}=96)$. Because we collected samples from each EU over time, we analyzed data using repeated measures mixed model ANOVA (Statistical Package SAS, v 9.2). To avoid pseudoreplication we took the mean of each response variable collected on each $\mathrm{EU}$ on each sampling date (i.e., the mean of 10 fish for proximate body composition, 5 for recent daily growth, 16 for morphology). The exception to this was water quality data, for which we had one data point per EU on each sample date (except the four sites in summer 2010, as discussed above). We used SLICES in the model to examine interaction effects to determine whether there were significant differences in the response after explanatory variables were incorporated into the model (i.e., marsh type, time, region, parasitism status, gravidity, sex). We used Heterogeneous Autoregressive (1) as our covariance structure because it assumes that data that are farther apart in time will be less similar and that each time period has its own unique variance. Assumptions of normality and equality of variances within datasets were verified prior to all statistical analyses. We arcsinesquare-root transformed our percent lipid, lean dry, and water data prior to analysis. For proximate body composition and growth rate data we incorporated mean fish length into the model as a covariate to ensure significant differences were attributable to marsh type and not differences in fish size [49]. Significance was determined at the $\alpha=0.05$ level. We developed figures and graphics using SigmaPlot (v. 9.0) and the $R$ statistical software environment (v. 2.14.1). 
Proportions of gravid and/or parasitized fish were compared between habitats using Two Sample Tests for Proportions; data is reported as the mean \pm proportional standard deviation. A continuity correction was conducted for the restricted vs. reference gravidity data to increase the quality of the normal approximation to the binomial distribution. To determine whether it was necessary to remove afflicted individuals from the analysis, we quantified the effects of parasitism and gravidity on fish lipid mass and morphology using repeated measures ANOVA. Due to unequal sample size $(>2 x)$, we analyzed the effects of parasitism/gravidity on recent daily growth using Welch's t-tests. We used Simple Linear Regression to model the relationship between fish length and otolith radius in healthy fish (i.e., those without ecto/endoparasites or eggs present) and examined homogeneity of fish age class distributions using Chi Square Tests of Homogeneity. Lipid and lean dry mass results are presented as a percentage of fish dry weight, water mass as a percent of wet weight, growth as the mean recent daily growth increment of the otolith (in micrometers), and morphology as a unitless index value $(\mathrm{K})$. Means are reported for each statistic \pm standard deviation.

\section{Results}

Field Data

Water Quality

We collected 164 sets of water quality data from the 48 stations from 2010-2011

(Table 3-2). In the restored vs. reference sites in Long Island Sound (LIS), we found no significant difference in salinity $\left(\mathrm{p}=0.9717 ; \mathrm{t}_{40}=0.04\right)$, temperature $\left(\mathrm{p}=0.4287 ; \mathrm{t}_{40}=-\right.$ 
$0.80)$, or dissolved oxygen $\left(\mathrm{p}=0.3747 ; \mathrm{t}_{40}=-0.90\right)$, which mirrored results in the Gulf of Maine (GOM; salinity: $\mathrm{p}=0.9542, \mathrm{t}_{40}=-0.06$; temperature: $\mathrm{p}=0.8690, \mathrm{t}_{40}=-0.17$;

dissolved oxygen: $\mathrm{p}=0.5496, \mathrm{t}_{40}=0.60$ ). In LIS, we found a highly significant difference in salinity between restricted vs. reference sites $\left(\mathrm{p}=0.0019 ; \mathrm{t}_{40}=3.33\right)$, but not for temperature $\left(\mathrm{p}=0.1588 ; \mathrm{t}_{40}=-1.44\right)$ or dissolved oxygen $\left(\mathrm{p}=0.3821 ; \mathrm{t}_{40}=-0.88\right)$, which also mirrored results in the GOM (salinity: $\mathrm{p}<0.0001, \mathrm{t}_{40}=11.89$; temperature: $\mathrm{p}=0.1409, \mathrm{t}_{40}=-1.50$; dissolved oxygen: $\mathrm{p}=0.2253, \mathrm{t}_{40}=-1.23$; Table $3-2$ ).

\section{Parasitism and Gravidity}

Fundulus heteroclitus were infected by a range of parasites including sea lice (Branchiura), anchor worms (Copepoda), flat worms (Monogenea, Digenea), internal cavity worms infecting the liver, intestines, and mesenteries (Cestoda, Acanthocephala), and the internal nematode parasite, Eustrongylides spp. We grouped data by parasite location (ecto/endo) and found that fish in the restricted marshes had the highest overall prevalence and weighted prevalence of parasite infection among the marsh types (Table 3-3). Overall infection intensity was also highest for the restricted marsh fish. We analyzed the proportion (prevalence) of parasitized fish by marsh type and found no significant difference between the reference $(n=62 / 755 ; 8.21$ $\pm 1.00 \%$ ) vs. restored marsh fish $(n=72 / 751 ; 9.59 \pm 1.07 \%$; $p=0.3486, \mathrm{Z}=-0.94$; Fig. 32; Table 3-3). However, we found significantly more parasitized fish in restricted marshes $(\mathrm{n}=185 / 756 ; 24.47 \pm 1.56 \%)$ in comparison to adjacent reference marshes $(\mathrm{n}=125 / 754 ; 16.58 \pm 1.35 \% ; \mathrm{p}=0.0001 ; \mathrm{Z}=-3.80 ;$ Fig. $3-2$; Table 3-3). Within the female population collected over our entire study period (2010-2011), there was no 
difference in the proportion of gravid fish in the reference $(\mathrm{n}=27 / 397 ; 6.80 \pm 1.26 \%)$ vs. restored marshes $(n=29 / 378 ; 7.67 \pm 1.37 \% ; p=0.6397 ; Z=-0.47 ;$ Fig. $3-2)$. However, we did find significantly fewer gravid fish inhabiting the restricted $(n=10 / 392 ; 2.55 \pm 0.80 \%)$ vs. reference marshes $(n=32 / 385 ; 8.31 \pm 1.41 \% ; p=0.0007$; $Z=3.55$; Fig. 3-2) from 2010-2011.

\section{Laboratory Data}

\section{$\underline{\text { Physiological Condition and Growth }}$}

\section{Proximate Body Composition (Lipid/Lean Dry/Water)}

We successfully extracted whole body lipids from 1,915 of 1,920 fish captured from 2010-2011. Approximately $14.67 \%(\mathrm{n}=281)$ of the fish analyzed for proximate body composition were parasitized. Incorporation of parasitism status into a repeated measures ANOVA revealed a significant negative effect on lipid stores when fish length was added as a covariate $\left(\mathrm{p}=0.0181 ; \mathrm{F}_{1,37}=6.12\right)$, with lower lipid reserves in parasitized fish $(\bar{x}=7.90 \pm 2.89 \%)$ than in unparasitized fish $(\bar{x}=8.44 \pm 2.55 \%)$. Approximately $6.84 \%(\mathrm{n}=68)$ of the fish analyzed for proximate body composition were gravid. The unparasitized gravid female fish had significantly less lipid than the non-gravid females $\left(p<0.0001 ; F_{1,20}=88.44\right)$. These effects were highly significant and consistent across marsh types, with gravid females averaging $4.89 \pm 1.92 \%$ lipid and non-gravid females averaging $8.33 \pm 2.04 \%$ lipid, indicating a significant allocation of energy reserves to reproduction. Since we found significant negative effects of parasitism and gravidity on lipid mass, we removed all gravid and afflicted fish from further analyses to eliminate confounding effects and clarify the interpretation of our 
results ( $\mathrm{n}=338 / 1,915$ removed; $17.65 \%)$. The fish in all subsequent lipid analyses represent unparasitized, non-gravid (termed "healthy") individuals in the population $(\mathrm{n}=1,577)$. A consequence, however, is that the mean from each EU became unbalanced (i.e., $\mathrm{n}<10$ ).

Using pooled data by sex across habitat/time periods, we found that fish in the Gulf of Maine had significantly more lipid than those in Long Island Sound $(\mathrm{p}<0.0001$; $F_{1,40}=125.70$ ), which was consistent by season and suggests influences of countergradient variation $[92,93]$. Overall, females contained more lipid than males $\left(\mathrm{p}=0.0001 ; \mathrm{F}_{1,40}=18.64 ;\right.$ Table $\left.3-4\right)$. We found significant differences overall by season $\left(\mathrm{p}<0.0001 ; \mathrm{F}_{3,120}=30.67\right)$, with fall fish (pre-hibernation) having significantly more lipid than summer fish (post-reproduction) in both $2010\left(\mathrm{p}=0.0008 ; \mathrm{t}_{120}=3.43\right)$ and $2011\left(p<0.0001 ; t_{120}=7.66\right.$; Table 3-4). By marsh type, we found no difference in the lipid mass of healthy fish inhabiting the restored vs. reference marshes $(\mathrm{p}=0.2445$; $\mathrm{t}_{40}=1.18$; Table 3-4; Fig. 3-3a). When we analyzed the interactions between marsh type, region, time, and sex we found a significant difference between the restored and reference habitats in LIS ( $\mathrm{p}=0.0278 ; \mathrm{t}_{40}=2.28$ ), which was likely driven by differences in males in fall $2010\left(\mathrm{p}=0.0129 ; \mathrm{t}_{40}=2.60\right)$. We found a highly significant difference in lipid mass between fish inhabiting the restricted vs. reference marshes $(\mathrm{p}=0.0013$; $\mathrm{t}_{40}=3.45$; Tables 3-4; 3-5; Fig. 3-3a). Significant differences between restricted and reference marsh fish held with comparisons of fish from the GOM, LIS, in three of the four time periods sampled, and for both males and females (Table 3-5). 
We analyzed lipid-free dry mass (composed primarily of protein and bone/ash) in healthy fish to examine investment in body structure vs. lipid storage. Because we analyzed data on a dry weight basis, $\%$ lipid and $\%$ lean dry mass are the only two proportions in dry fish weight. Therefore, the statistics reported (Table 3-5) are nearly identical, but in the opposite direction. Overall, lean dry mass constituted a lower proportion of fish body weight in the GOM than in LIS $\left(p<0.0001 ; \mathrm{F}_{1,40}=125.70\right)$ and lean dry mass in females was lower than that of males $\left(p=0.0001 ; F_{1,40}=18.64\right.$; Table 3-4). By marsh type, we found no difference between the restored and reference sites in the proportion of mass allocated to structure $\left(\mathrm{p}=0.2445 ; \mathrm{t}_{40}=-1.18\right)$ or water $\left(p=0.6547 ; t_{40}=-0.45 ;\right.$ Table $3-4$; Figs. $\left.3-3 b, c\right)$. We found a highly significant difference between the restricted and reference sites in the proportion allocated to structural mass $\left(\mathrm{p}=0.0013 ; \mathrm{t}_{40}=-3.45\right)$ but not for water mass $\left(\mathrm{p}=0.5213 ; \mathrm{t}_{40}=-0.65\right.$; Tables 3-4; 3-5; Figs. 3-3b,c). We also found no difference in water mass by region $\left(p=0.0826 ; F_{1,40}=3.17\right)$ and for most of the interactions (Table 3-5).

\section{Fish Age and Recent Daily Growth Rate}

Our capture and fish selection methodology was designed to gather information from a range of fish sizes present at each site, so we analyzed whether the proportion of age classes differed between marsh systems. We report age data from 465 fish in 2010 and 479 fish in 2011. From 2010-2011, we captured five age classes of fish (ages 0, 1, 2 , $3,4)$. Although it was not our intent to capture fish in the age 0 class (i.e., those in their first year of life), we captured 31 fish in fall 2011 that had grown to at least $40 \mathrm{~mm}$ and were therefore included in our field collections. Chi Square Tests of 
Homogeneity revealed a significant difference in age class distributions between restored vs. reference marsh systems $\left(p=0.0280 ; \chi_{4}^{2}=10.8785 ; n=473\right.$; Fig. $\left.3-4\right)$ but not between the restricted vs. reference marshes $\left(\mathrm{p}=0.3643 ; \chi^{2}{ }_{4}=4.3211 ; \mathrm{n}=471\right.$; Fig. 3-4). Within the four marsh types, the frequency of the smallest age classes (ages 0 and 1) was highest in reference marshes adjacent to restored sites $(\mathrm{n}=78 ; 32.77 \%)$ and lowest in the tidally restricted marshes $(n=33 ; 14.04 \%)$. Fish in the other two marsh groups were intermediate (restored: 56 fish, 23.83\%; reference adjacent to restricted: 47 fish 19.92\%; Fig. 3-4). Therefore, reference marshes adjacent to restored marshes harbored the largest proportion of young fish.

Due to unclear daily growth rings or other structural abnormalities in the otoliths (e.g., irregular accretion of calcium carbonate along the edge, resulting in a scalloped morphology) we initially discarded 263 fish from our study, with an additional 155 discards due to a $>10 \%$ difference between the first and second growth readings. In total we analyzed growth rate data from 542 fish from 2010-2011 (56.5\%). Our approach is consistent with other studies that have selected only the clearest otoliths for microstructure analysis (top 15.7\%) [91] or discarded data from up to $44.9 \%$ of samples due to imprecise increment patterns, accessory primordia, or errors during sample preparation [89,92-94].

An analysis of the effects of parasitism and gravidity did not reveal significant negative effects on fish growth rate $\left(\mathrm{p}=0.7739 ; \mathrm{t}_{94.26}=-0.288\right)$; however, we removed an additional 81 parasitized and/or gravid individuals from the growth rate analysis to 
be consistent in our interpretation of results across physiological and morphological analyses, resulting in growth rate data for 461 healthy fish. Using simple linear regression we found a highly significant relationship between fish length and otolith radius for healthy fish $\left(\mathrm{p}<0.0001 ; \mathrm{r}^{2}=0.6628\right.$; Otolith radius $=-2.77341+0.09572 *$ fish length; Fig. 3-5). Therefore, the marginal ten increments of $F$. heteroclitus otoliths can be used as a reliable indicator of recent daily growth at our sites.

Using the healthy individuals in the population and fish length as a covariate, we found that females grow significantly faster than males $\left(\mathrm{p}=0.0461 ; \mathrm{F}_{1,38}=4.25\right.$; Table 3-6), so we separated our model by sex. Unlike our lipid mass results, we found no difference in the growth rate of fish residing in the GOM vs. LIS $(p=0.2786$; $\left.\mathrm{F}_{1,40}=1.21\right)$. However, we did find a significant effect of season in the marshes, with fish growing at a higher rate in summer than in fall in both $2010\left(\mathrm{p}<0.0001 ; \mathrm{t}_{93}=-\right.$ 13.63) and $2011\left(\mathrm{p}<0.0001 ; \mathrm{t}_{93}=-8.58\right.$; Table 3-6). The higher growth rate in 2010 across habitats, regions, and seasons corresponds generally to a lower investment in energy storage (Tables 3-4, 3-6), whereas in 2011 the relationship is reversed (lower growth rate, higher energy investment), indicating potential trade-offs in somatic investments that may shift from year to year. Seasonally, fish in the summer are growing faster but have depleted lipid stores, whereas in fall the fish are growing slower but have significantly higher lipid reserves.

By marsh type, we did not detect differences in the growth rate between fish residing in restored vs. reference marshes $\left(\mathrm{p}=0.2506 ; \mathrm{t}_{40}=1.17\right)$, nor between fish in the 
restricted vs. reference marshes $\left(\mathrm{p}=0.5153 ; \mathrm{t}_{40}=0.66\right.$; Table $\left.3-6\right)$. However, we did detect a difference in growth rate between the restored and reference sites within the LIS region $\left(\mathrm{p}=0.0389 ; \mathrm{t}_{40}=2.14\right)$ that mirrors our proximate body composition data. The difference in LIS appears to be driven by the males $\left(\mathrm{p}=0.0201 ; \mathrm{t}_{38}=2.43\right)$ rather than the females, which were equivalent between marsh types $\left(\mathrm{p}=0.5327 ; \mathrm{t}_{38}=0.63\right)$. For the restricted vs. reference fish, none of the interactions for growth rate by season, region, and time were significant ( $\mathrm{p}>0.05$ ), indicating that fish of similar lengths are growing at the same rate in both the restricted and reference habitats despite the differences in allocation of resources to lipid storage (Tables 3-4, 3-6).

\section{$\underline{\text { Morphological Condition }}$}

\section{Fulton's Condition Factor (K)}

Analysis of morphology data using Fulton's K in our repeated measures ANOVA revealed no overall negative effect of parasitism/gravidity on fish condition $\left(\mathrm{p}=0.7453 ; \mathrm{F}_{1,40}=0.11\right)$. However, to be consistent in our interpretation of results across analyses we removed all afflicted individuals from the analysis $(\mathrm{n}=517)$. Using only healthy fish $(\mathrm{n}=2,499)$, we found no significant difference between the restored vs. reference $\left(\mathrm{p}=0.6273 ; \mathrm{t}_{40}=-0.49\right)$ or the restricted vs. reference marsh fish $\left(p=0.4962 ; t_{40}=0.69\right)$. Analysis of possible interactions between marsh type, region, time, and sex revealed only one significant difference between the reference and restricted marsh fish in fall $2010\left(\mathrm{p}=0.0458 ; \mathrm{t}_{120}=2.02\right)$, with Fulton's $\mathrm{K}$ indicating that reference marsh fish were in better condition than those in restricted marshes. We did find a difference between the summer and fall seasons in $2010\left(p=0.0016 ; t_{120}=-3.22\right)$ 
and a marginal difference in $2011\left(\mathrm{p}=0.0570 ; \mathrm{t}_{120}=-1.92\right)$, but the effect was in the opposite direction, with Fulton's K labeling summer fish (post-reproduction) healthier than those in fall (pre-hibernation) in both years. In addition, this morphological index did not detect trends in condition between sexes $\left(\mathrm{p}=0.3804 ; \mathrm{F}_{1,40}=0.89\right)$ or regions $\left(p=0.7849 ; F_{1,40}=-0.27\right)$ found using physiological indices.

\section{Length-Weight Relationships}

We analyzed length-weight relationships using Multiple Linear Regression (with categorical variables for the marsh types). Examination of fit statistics (AIC, AICC, BIC), output from the regression coefficient hypothesis tests, adjusted $\mathrm{R}^{2}$, and multicollinearity statistics (tolerance, variance inflation factor) revealed that quadratic models best explained the length-weight relationships for the restored, restricted, and reference marsh fish (Figs. 3-6a,b). The restored and reference fish populations were best explained by one line with the following equation $\left(\operatorname{adj} . \mathrm{R}^{2}=0.9691 ; \mathrm{p}<0.0001\right.$; Fig. 3-6a):

Fish mass $=1.52097-0.11079($ fish length $)+0.00218(\text { fish length })^{2}$ There was a strong positive linear relationship $(\mathrm{p}=0.0058)$ as well as evidence of a curvilinear relationship $(\mathrm{p}<0.0001)$ between fish length and weight, with the intercept not significantly different from zero $(\mathrm{p}=0.2329)$. For the restricted vs. reference marsh

fish, one regression line again best explained both populations (adj. $\mathrm{R}^{2}=0.9602$; p<0.0001; Fig. 3-6b):

Fish mass $=3.04263-0.16490($ fish length $)+0.00267(\text { fish length })^{2}$ 
There was a strong positive linear relationship $(\mathrm{p}=0.0009)$ and evidence of a curvilinear relationship $(\mathrm{p}<0.0001)$ between fish length and weight, with no difference in intercept ( $\mathrm{p}=0.0668)$. Combined with results using the Fulton's K condition factor, results indicate that fish at our study sites are morphologically indistinguishable.

\section{Discussion}

Tradeoffs between Fish Growth, Energy Storage, and Reproduction

Our study demonstrates that fish residing in tidally restricted marshes invaded by $P$. australis allocate a greater proportion of resources to maintaining growth and body size than to building lipid stores relative to reference marsh fish. Results were consistent by gender, region, and for three of the four seasons sampled. Our findings suggest potential tradeoffs between growth, energy storage, and reproduction potentially due to reduced habitat quality, a decrease in access to invertebrate prey on the marsh surface, and lack of habitat refugia to avoid larger predators.

Access to the marsh surface is ultimately influenced by the frequency, depth, and duration of tidal flooding, with nekton exhibiting a positive relationship between marsh selection and flooding duration [6]. Although we did not collect data on marsh surface flooding at our sites, we collected samples on flood tide and only observed flooding of $P$. australis at the fringe of one of the four restricted sites (Herring River), whereas all reference and restored marshes flooded daily on high tides. Flooding of the marsh surface in invaded tidally restricted salt marshes is influenced by both the reduction in tidal range due to the restriction (Table 3-1) and by the increase in marsh 
surface elevation due to introduced $P$. australis [95]. At one of our study sites

(Hatches Harbor), Smith et al. [35] measured tidal range pre-restoration and found that tidal range in the restricted marsh was only $39 \%$ of that measured in the adjacent unrestricted marsh. At another one of our sites (Galilee), the depth and duration of flooding in the restricted marsh increased post-restoration, whereas the reference marsh remained the same [25]. Osgood et al. [96] found that a $P$. australis-invaded (unrestricted) marsh in Connecticut was $29.0 \mathrm{~cm}$ higher in elevation than an adjacent S. alterniflora marsh, resulting in a reduction in flooding frequency by $52 \%$. Similarly, Hunter et al. [97] documented declines in marsh surface flooding depth from the initial $(6.0 \pm 0.5 \mathrm{~cm})$, early $(3.9 \pm 1.3 \mathrm{~cm})$, to late $(2.4 \pm 0.8 \mathrm{~cm})$ invasion stages that corresponded with reductions in flooding frequency by $7 \%, 16 \%$, and $37 \%$, respectively, in three $P$. australis-invaded (unrestricted) salt marshes in the midAtlantic region.

Our data suggest that with reduced or limited access to the marsh surface, $F$. heteroclitus in tidally restricted marshes invaded by $P$. australis are not obtaining dietary prey items needed to supplement their energy intake. Invertebrate prey on the marsh surface can differ than those typically found in subtidal creeks, with the former composed of isopods, gastropods, insects, spiders, beetles, amphipods, and ostracods and the latter composed of copepods, amphipods, and polychaetes [98]. The guts of fish allowed access to marsh surface can be up to six times fuller than those restricted to unvegetated subtidal creeks [98], providing resources necessary for significantly higher growth rates and weight gain $[4,12,14,15]$. In unrestricted $P$. australis marshes 
in the Hudson River estuary, Weinstein et al. $[16,99]$ reported reductions in the energy reserves (triacylglycerols, free fatty acids, total lipids) of fish, which they attributed to reduced flooding frequency and access to the marsh surface for feeding. Therefore, decreased lipid reserves detected in our study could be due to lack of fish access to invertebrate prey on the marsh surface.

A second potential reason for reduced lipid reserves relates to increased movement of fish due to predation risk and reduced habitat refugia at high tide. For F. heteroclitus, predation risk is of primary importance in determining habitat use [45,46,100]. At low tide, F. heteroclitus will occupy depositional areas of water channels where prey is less abundant but predation pressure is low [45]. When the tide rises, fish flood onto the marsh surface to feed and escape predators [45], with adult F. heteroclitus moving farther onto the marsh surface than juveniles, which stay near the marsh fringe [19]. Increased risk of predation could confine movement of $F$. heteroclitus to areas with poor prey availability [45], decrease growth rates [46], or increase movements to avoid capture from predatory fish and wading birds [101,102], thereby decreasing resources available for energy storage.

We found gravidity in F. heteroclitus strongly influenced their lipid reserves. Not only did we detect a significant cost of reproduction in $F$. heteroclitus (as evidenced by reduced lipid stores in unparasitized gravid females), the decreased proportion of gravid fish in restricted marshes suggests that investment tradeoffs between growth, lipid storage, and reproduction are occurring in the restricted marsh fish. Competing 
demands for energy acquisition, avoidance of predators while foraging, parasitism, and coping with seasonal fluctuations in north temperate estuaries influence energy allocation strategies in fish [49-51,61]. Notably, we did not find any differences in growth rate or morphology between the restricted, restored, or reference marsh fish, indicating investment into growth is a high priority across all populations.

Reproduction is costly [50,92], so fish may choose to skip spawning and invest resources into growth and survival to enhance the chance of future success rather than deplete current lipid stores by spawning [50]. Whether decreased lipid reserves in unparasitized fish inhabiting restricted marshes were due to decreased foraging ability, increased movement due to predation, or some other factor, it appears that investment into lipid stores has been forgone in lieu of growth.

\section{Effectiveness of Tidal Restoration}

Restoring hydrologic flow to salt marshes to decrease the cover and height of introduced $P$. australis has been a standard restoration practice in New England for decades and is used to re-establish habitat quality for salt marsh nekton and birds [24,30]. Previous authors in New England have examined hydrologic restoration effectiveness using gut content analyses, nekton density, length frequency distributions, fish biomass, and species richness/diversity, with varying outcomes based on restoration longevity, tidal range, site location, species, and metric assessed $[25,34,36,37,40,43,44,67,71,77,103]$. Our results support the effectiveness of tidal restoration for nekton, as all environmental, physiological, and morphological indices 
revealed that hydrologically restored marshes were equivalent in habitat quality for fish relative to adjacent reference systems.

Notably, fish using the reference S. alterniflora marshes were smaller in length than those within the restored marshes, likely because we captured a significantly larger abundance of younger individuals (ages 0-1) in the reference marshes. Intertidal salt marshes serve as nurseries for young $F$. heteroclitus $[3,4,19,100]$, which use small surface marsh pools and depressions for feeding and refuge during their first summer until they have obtained sufficient length to enter the tidal creek system [32,33,96,97]. Many of our restored sites are still changing and have yet to develop an extensive network of pools typical of salt marshes, so exposure of juveniles to predators may be higher than in reference marshes. Adult $F$. heteroclitus are known to consume their younger conspecifics so it possible that young-of-the-year fish are fewer in number in restored marshes simply due to predation $[33,102]$.

Over time, nekton patterns in the restored marshes can mimic those in reference areas as the hydrologic connection between habitats allows greater faunal and prey exchange $[34,37,40,44]$. Our results demonstrate that restored and reference salt marshes are equivalent in their provision of habitat to resident salt marsh fish as indicated by non-significant differences in energy reserves, growth rate, morphology, gravidity, parasite prevalence, and water quality 11-22 years post-restoration. The outcomes of our study agree with the findings of two recent meta-analyses (one global, one regional) that concluded that in degraded wetlands ecological restoration 
of faunal communities can rapidly occur within the timeframe of 5-10 years, especially where there is a hydrologic connection to an intact marsh system [104, K.L. Dibble, unpublished data]. Although other wetland functions such carbon storage and nutrient cycling may take many more years to achieve [104], habitat quality for fauna can be restored relatively quickly in these systems.

\section{Conclusions}

Tidally restricted salt marshes invaded by introduced $P$. australis have been the focus of restoration efforts due to measurable differences in biodiversity and ecosystem function. We demonstrated that fish in restricted, restored and reference marshes are morphologically similar so that an assessment of condition based on fish length or biomass might not capture the physiological effects of poor habitat quality on resident

fish populations. Instead, we used biochemical condition indices and examined parasites and gravidity and were able to detect trends in the health of a common marsh resident. Numerically dominant along the Atlantic coast, $F$. heteroclitus consume salt marsh herbivores/detritivores and are prey to transient predators, thereby providing an important trophic link between intertidal marsh production and near- and offshore food webs [102]. Management efforts to restore tidal exchange and control the $P$. australis invasion in salt marshes should be a priority to ensure that forage fish populations are healthy and can support coastal fisheries.

\section{Acknowledgements}

We thank Penelope Pooler for her assistance with and review of our analysis and 
results. We also thank Peter August and the University of Rhode Island Environmental

Data Center for drafting the map of our study sites.

\section{References}

1. Boesch DF, Turner RE (1984) Dependence of fishery species on salt marshes: the role of food and refuge. Estuaries 7(4A): 460-468.

2. Taylor MH (1986) Environmental and endocrine influences on reproduction of Fundulus heteroclitus. Am Zool 26(1): 159-171.

3. Beck MW, Heck KL Jr., Able KW, Childers DL, Eggleston DB, et al. (2001) The identification, conservation, and management of estuarine and marine nurseries for fish and invertebrates. Bioscience 52(8): 633-641.

4. Minello TJ, Able KW, Weinstein MP, Hays CG (2003) Salt marshes as nurseries for nekton: testing hypotheses on density, growth, and survival through metaanalysis. Mar Ecol Prog Ser 246: 39-59.

5. Balouskus RG, Targett TE (2012) Egg deposition by Atlantic silverside, Menidia menidia: substrate utilization and comparison of natural and altered shoreline type. Estuaries Coast 35: 1100-1109.

6. Minello TJ, Rozas LP, Baker R (2012) Geographic variability in salt marsh flooding patterns may affect nursery value for fishery species. Estuaries Coast 35: 501-514.

7. Teal JM (1962) Energy flow in the salt marsh ecosystem of Georgia. Ecology 43:614-24.

8. Darnell RM (1967) Organic detritus in relation to the estuarine ecosystem. In: Lauff GH (ed) Estuaries. AAAS Publ 83, Washington, DC, p 376-382.

9. Odum EP (1968) A research challenge: evaluating the productivity of coastal and estuarine water. Proceedings, 2nd Sea Grant Conference, Graduate School of Oceanography. University of Rhode Island, Kingston, Rhode Island.

10. Nixon SW (1980) Between coastal marshes and coastal waters: a review of twenty years of speculation and research on the role of salt marshes in estuarine productivity and water chemistry. Pages 437-525 in Hamilton P, MacDonald KB, eds. Estuarine and Wetland Processes. New York: Plenum Press. 
11. Childers DL, Day JW Jr., McKellar HN Jr. (2000) Twenty more years of marsh and estuarine flux studies: revisiting Nixon (1980). In Concepts and controversies in tidal marsh ecology. Edited by M.P. Weinstein and D.A. Kreeger. Kluwer Academic Publishers, Dordrecht, the Netherlands. pp. 391-424.

12. Weisberg SB, Lotrich VA (1982) The importance of an infrequently flooded intertidal marsh surface as an energy source for the mummichog Fundulus heteroclitus: an experimental approach. Mar Biol 66: 307-10.

13. Irlandi EA, Crawford MK (1997) The effect of intertidal saltmarshes and adjacent subtidal habitats on abundance, movement, and growth of an estuarine fish. Oecologia 110(2): 222-230.

14. Javonillo R, Deegan L, Chiaravalle K, and Hughes J (1997) The importance of access to salt marsh surface to short-term growth of Fundulus heteroclitus in a New England salt marsh. Biol Bull 193: 288-289.

15. Madon SP, Williams GD, West JM, and Zedler JB (2001) The importance of marsh access to growth of the California killifish, Fundulus parvipinnis, evaluated through bioenergetics modeling. Ecol Modell 135: 149-165.

16. Weinstein MP, Litvin SY, and Guida VG (2009) Essential Fish Habitat and wetland restoration success: A tier III approach to the biochemical condition of common mummichog Fundulus heteroclitus in common reed Phragmites australis- and smooth cordgrass Spartina alterniflora- dominated salt marshes. Estuaries Coast 32: 1011-1022.

17. Valiela I, Wright JE, Teal JM, Volkmann SB (1977) Growth, production, and energy transformations in the salt marsh killifish Fundulus heteroclitus. Mar Biol 40: $135-144$.

18. Weisberg SB, Whalen R, Lotrich VA (1981) Tidal and diurnal influence on food consumption of a salt marsh killifish Fundulus heteroclitus. Mar Biol 61: 243246.

19. Kneib RT, Wagner SL (1994) Nekton use of vegetated marsh habitats at different stages of tidal inundation. Mar Ecol Prog Ser 106: 227-238.

20. Watzin MC, Gosselink JG (1992) The fragile fringe: coastal wetlands of the continental United States. Louisiana Sea Grant College Program, Louisiana State University, Baton Rouge, LA; U.S. Fish and Wildlife Service, Washington, DC; and National Oceanic and Atmospheric Administration, Rockville, MD. 19pp.

21. Gedan KB, Silliman BR, Bertness MD (2009) Centuries of human-driven change in salt marsh ecosystems. Annu Rev Mar Sci 1: 117-141. 
22. Weis JS, Butler CA (2009) Salt marshes: A natural and unnatural history. New Jersey: Rutgers University Press. 254 p.

23. Portnoy JW, Giblin AE (1997) Effects of historic tidal restrictions on salt marsh sediment chemistry. Biogeochemistry 36: 275-303.

24. Roman CT, Niering WA, Warren RS (1984) Salt marsh vegetation change in response to tidal restriction. Environ Manage 8: 141-150.

25. Raposa K (2002) Early responses of fishes and crustaceans to restoration of a tidally restricted New England salt marsh. Restor Ecol 10(4): 665-676.

26. Chambers RM, Meyerson LM, Saltonstall K (1999) Expansion of Phragmites australis into tidal wetlands of North America. Aquat Bot 64: 261-73.

27. Chambers RM, Osgood DT, Bart DJ, Montalto F (2003) Phragmites australis invasion and expansion in tidal wetlands: interactions among salinity, sulfide, and hydrology. Estuaries 26(2B): 398-406.

28. Silliman BR, Bertness MD (2004) Shoreline development drives invasion of Phragmites australis and the loss of plant diversity on New England salt marshes. Conserv Biol 18(5): 1424-34.

29. Meyerson LA, Saltonstall K, Windham L, Kiviat E, Findlay S (2000) A comparison of Phragmites australis in freshwater and brackish marsh environments in North America. Wetl Ecol Manag 8: 89-103.

30. Chambers RM, Meyerson LA, Dibble KL (2012) Ecology of Phragmites australis and responses to tidal restoration. In: Roman C, Burdick D, editors. Tidal marsh restoration: A synthesis of science and management. Washington, DC: Island Press.

31. Rooth JE, Court Stevenson J, Cornwell JC (2003) Increased sediment accretion rates following invasion by Phragmites australis: the role of litter. Estuaries 26(2B): 475-83.

32. Able KW, Hagan SM, Brown SA (2003) Mechanisms of Marsh Habitat Alteration Due to Phragmites: response of Young-of-the-year Mummichog (Fundulus heteroclitus) to Treatment for Phragmites Removal. Estuaries 26(2B): 484-494.

33. Able KW, Hagan SM, Brown SA (2006) Habitat use, movement, and growth of young-of-the-year Fundulus spp. in southern New Jersey salt marshes: comparisons based on tag/recapture. J Exp Mar Biol Ecol 335: 177-187. 
34. Buchsbaum RN, Catena J, Hutchins E, James-Pirri MJ (2006) Changes in salt marsh vegetation, Phragmites australis, and nekton in response to increased tidal flushing in a New England salt marsh. Wetlands 26(2): 544-557.

35. Smith SM, Roman CT, James-Pirri MJ, Chapman K, Portnoy J, et al. (2009) Responses of plant communities to incremental hydrologic restoration of a restricted salt marsh in southern New England (Massachusetts, U.S.A.). Restor Ecol 17(5): 606-618.

36. Raposa KB (2008) Early ecological responses to hydrologic restoration of a tidal pond and salt marsh complex in Narragansett Bay, Rhode Island. J Coast Res 55: $180-92$.

37. Roman CT, Raposa KB, Adamowicz SC, James-Pirri MJ, Catena JG (2002) Quantifying vegetation and nekton response to tidal restoration of a New England salt marsh. Restor Ecol 10(3): 450-60.

38. Benoit LK, Askins RA (1999) Impact of the spread of Phragmites on the distribution of birds in Connecticut tidal marshes. Wetlands 19(1): 194-208.

39. Raposa KB, Roman CT (2001) Seasonal habitat-use patterns of nekton in a tiderestricted and unrestricted New England salt marsh. Wetlands 21(4): 451-461.

40. Eberhardt AL, Burdick DM, and Dionne M (2011) The effects of road culverts on nekton in New England salt marshes: implications for tidal restoration. Restor Ecol 19(6): 776-785.

41. Anisfeld SC (2012) Biogeochemical responses to tidal restoration. In: Roman C, Burdick D, editors. Tidal marsh restoration: A synthesis of science and management. Washington, DC: Island Press.

42. Smith SM and Warren RS (2012) Vegetation responses to tidal restoration. In: Roman C, Burdick D, editors. Tidal marsh restoration: A synthesis of science and management. Washington, DC: Island Press.

43. Raposa KB, Talley DM (2012) A meta-analysis of nekton responses to restoration of tide-restricted New England salt marshes. In: Roman C, Burdick D, editors. Tidal marsh restoration: A synthesis of science and management. Washington, DC: Island Press.

44. James-Pirri MJ, Raposa KB, Catena JG (2001) Diet composition of mummichogs, Fundulus heteroclitus, from restoring and unrestricted regions of a New England (U.S.A.) salt marsh. Estuar Coast Shelf S 53: 205-213.

45. McIvor CC, Odum WE (1988) Food, predation risk, and microhabitat selection in a marsh fish assemblage. Ecology 69(5): 1341-1351. 
46. Halpin PM (2000) Habitat use by an intertidal salt marsh fish: trade-offs between predation and growth. Mar Ecol Prog Ser 198: 203-214.

47. Lloret J, Planes S (2003) Condition, feeding and reproductive potential of white seabream Diplodus sargus as indicators of habitat quality and the effect of reserve protection in the northwestern Mediterranean. Mar Ecol Prog Ser 248: 197-208.

48. Amara R, Selleslagh J, Billon G, Minier C (2009) Growth and condition of 0group European founder, Platichthys flesus as indicator of estuarine habitat quality. Hydrobiologia 627: 87-98.

49. Post JR, Parkinson EA (2001) Energy allocation strategy in young fish: allometry and survival. Ecology 82(4): 1040-1051.

50. Jorgensen C, Ernande B, Fiksen O, Dieckmann U (2006) The logic of skipped spawning in fish. Can J Fish Aquat Sci 63: 200-11.

51. Adams SM (1999) Ecological role of lipids in the health and success of fish populations. In: Arts MT, Wainman BC, editors. The ecological role of lipids in freshwater ecosystems. New York: Springer-Verlag.

52. Simpkins DG, Hubert WA, Martinez del Rio C, Rule DC (2003) Interacting effects of water temperature and swimming activity on body composition and mortality of fasted juvenile rainbow trout. Can J Zool 81: 1641-1649.

53. Lafferty KD (2008) Ecosystem consequences of fish parasites. J Fish Biol 73: 2083-2093.

54. Goto D, Wallace WG (2010) Bioenergetic responses of a benthic forage fish (Fundulus heteroclitus) to habitat degradation and altered prey community in polluted salt marshes. Can J Fish Aquat Sci 67: 1566-1584.

55. Fulton TW (1904) The rate of growth of fishes. $22^{\text {nd }}$ Annual Report of the Fishery Board of Scotland 1904(3): 141-241.

56. Campana SE, Neilson JD (1985) Microstructure of fish otoliths. Can J Fish Aquat Sci 42: 1014-1032.

57. Gilliers CR, Amara R, Bergeron JP, LePape O (2004) Comparison of growth and condition indices of juvenile flatfish in different coastal nursery grounds. Environ Biol Fishes 71: 189-198.

58. Kerambrun E, Le Floch S, Sanchez W, Guyon HT, Meziane T, Henry F, Amara R (2012) Responses of juvenile sea bass, Dicentrarchus labrax, exposed to acute concentrations of crude oil, as assessed by molecular and physiological 
biomarkers. Chemosphere 87: 692-702.

59. Fritz ES, Garside ET (1975) Comparison of age composition, growth, and fecundity between two populations each of Fundulus heteroclitus and $F$. diaphanous (Pisces: Cyprinodontidae). Can J Zool 53(4): 361-369.

60. Spalding MG, Bancroft GT, Forrester DJ (2003) The epizootiology of Eustrongylidosis in wading birds (Ciconiiformes) in Florida. J Wildlife Dis 29(2): 237-249.

61. Marcogliese DJ (2004) Parasites: small players with crucial roles in the ecological theater. EcoHealth 1: 151-164.

62. Marcogliese DJ (2005) Parasites of the superorganism: are they indicators of ecosystem health? Int J Parasitol 35: 705-716.

63. Sinicrope TL, Hine PG, Warren RS, Niering WA (1990) Restoration of an impounded salt marsh in New England. Estuaries 13(1): 25-30.

64. Milone and MacBroom, Inc. (1996) Sybil Creek salt marsh restoration Branford, CT, preliminary design report. Report to the Connecticut Department of Environmental Protection, Office of Long Island Sound Programs, September 1996, 85pp.

65. Anisfeld SC, Benoit G (1997) Impacts of flow restrictions on salt marshes: an instance of acidification. Environ Sci Technol 31: 1650-1657.

66. Anisfeld SC, Tobin MJ, Benoit G (1999) Sedimentation rates in flow-restricted and restored salt marshes in Long Island Sound. Estuaries 22(2A): 231-244.

67. Burdick DM, Dionne M, Boumans RM, Short FT (1997) Ecological responses to tidal restorations of two northern New England salt marshes. Wetl Ecol Manag 4(2): 129-144.

68. Brawley AH, Warren RS, Askins RA (1998) Bird use of restoration and reference marshes within the Barn Island Wildlife Management Area, Stonington, Connecticut, USA. Environ Manage 22(4): 625-633.

69. Slason E, Coron CR (2000) Impact of landfill leachate on a restricted tidal marsh of the Sybil Creek watershed, Branford, CT. Proceedings of the $5^{\text {th }}$ Biennial Long Island Sound Research Conference. Connecticut Sea Grant Publication CTSG-0102.

70. Boumans RMJ, Burdick DM, Dionne M (2002) Modeling habitat change in salt marshes after tidal restoration. Restor Ecol 10(3): 543-555. 
71. Warren RS, Fell PE, Rozsa R, Brawley AH, Orsted AC, Olson ET, Swamy V, Niering WA (2002) Salt marsh restoration in Connecticut: 20 years of science and management. Restor Ecol 10(3): 497-513.

72. Portnoy J, Roman C, Smith S, Gwilliam E (2003) Estuarine habitat restoration at Cape Cod National Seashore: the Hatches Harbor prototype. ParkScience 22(1): $51-58$.

73. Wozniak AS, Roman CT, Wainright SC, McKinney RA, and James-Pirri MJ (2006) Monitoring food web changes in tide-restored salt marshes: a carbon stable isotope approach. Estuaries Coast 29(4): 568-578.

74. Adamowicz SC, O’Brien K (2012) Drakes Island tidal restoration: science, community, and compromise. In: Roman C, Burdick D, editors. Tidal marsh restoration: A synthesis of science and management. Washington, DC: Island Press.

75. Golet FC, Myshrall DHA, Oliver LR, Paton PWC, Tefft BC (2012) Role of science and partnerships in salt marsh restoration at the Galilee Bird Sanctuary, Narragansett, Rhode Island. In: Roman C, Burdick D, editors. Tidal marsh restoration: A synthesis of science and management. Washington, DC: Island Press.

76. National Oceanic and Atmospheric Administration (2003) Drakes Island Marsh Restoration, Wells, Maine. Community-Based Restoration Program Fact Sheet.

77. Raposa KB, Roman CT (2003) Using gradients in tidal restriction to evaluate nekton community responses to salt marsh restoration. Estuaries 28(1): 98-105.

78. McMahon KW, Johnson BJ, Ambrose WG Jr (2005) Diet and movement of the killifish, Fundulus heteroclitus, in a Maine salt marsh assessed using gut contents and stable isotope analyses. Estuaries 28(6): 966-973.

79. Raposa KB, Roman CT, and Heltshe JF (2003) Monitoring nekton as a bioindicator in shallow estuarine habitats. Environ Monit Assess 81: 239-255.

80. Fry B, Cieri M, Hughes J, Tobias C, Deegan LA, Peterson B (2008) Stable isotope monitoring of benthic-planktonic coupling using salt marsh fish. Mar Ecol Prog Ser 369: 193-204.

81. Bush AO, Lafferty KD, Lotz JM, Shostak AW (1997) Parasitology meets ecology on its own terms: Margolis et al. revisited. J Parasitol 83(4): 575-583.

82. Harris CE, Vogelbein WK (2006) Parasites of mummichogs, Fundulus heteroclitus, from the York River, Virginia, U.S.A., with a checklist of parasites of Atlantic Coast Fundulus spp. Comp Parasitol 73(1): 72-110. 
83. Dobush GR, Ankney CD, Krementz DG (1985) The effect of apparatus, extraction time, and solvent type on lipid extractions of snow geese. Can J Zool 63: 1917-1920.

84. Radtke RL, Dean JM (1982) Increment formation in the otoliths of embryos, larvae, and juveniles of the mummichog, Fundulus heteroclitus. Fish Bull 80(2): 201-215.

85. Secor DH, Dean JM, Laban EH (1991) Manual for otolith removal and preparation for microstructural examination. Belle W. Baruch Institute for Marine Biology and Coastal Research, Technical Publication number 1991-01. 84pp.

86. Mugiya Y (1987) Effects of photoperiods on the formation of otolith increments in the embryonic and larval rainbow trout Salmo gairdneri. Nippon Suisan Gakk 53(11): 1979-1984.

87. Tomas J, Panfili J (2000) Otolith microstructure examination and growth patterns of Vinciguerria nimbaria (Photichthyidae) in the tropical Atlantic Ocean. Fish Res 46: 131-145.

88. Neilson JD (1992) Sources of error in otolith microstructure examination. In: Stevenson DK, Campana SE, editors. Otolith microstructure examination and analysis. Can Spec Publ Fish Aquat Sci 117: 115-125.

89. Parrish DL, Vondracek B, Eckmayer WJ (1994) Accuracy and precision of daily age estimates for walleyes from Ohio hatcheries and Lake Erie. Trans Am Fish Soc 123: 601-605.

90. Arneri E, Morales-Nin B (2000) Aspects of the early life history of European hake from the central Adriatic. J Fish Biol 56: 1368-1380.

91. Otxotorena U, Diez G, Lopez de Abechuco E, Santurtun M, Lucio P (2010) Estimation of age and growth of juvenile hakes (Merluccius merluccius Linneaus, 1758) of the Bay of Biscay and Great Sole by means of the analysis of macro and microstructure of the otoliths. Fish Res 106: 337-343.

92. Hassager TK (1991) Comparison of three different otolith-based methods for age determination of turbot (Scophthalmus maximus). Dana 9: 39-43.

93. Kelly CJ, Connolly PL, Bracken JJ (1999) Age estimation, growth, maturity, and distribution of the bluemouth rockfish Helicolenus $d$. dactylopterus (Delaroche 1809) from the Rockall Trough. ICES J Mar Sci 56: 61-74. 
94. Pineiro C, Rey J, de Pontual H, Garcia A (2008) Growth of Northwest Iberian juvenile hake estimated by combining sagittal and transversal otolith microstructure analysis. Fish Res 93: 173-178.

95. Burdick DM and Roman CT (2012) Salt marsh responses to tidal restriction and restoration: A summary of experiences. In: Roman C, Burdick D, editors. Tidal marsh restoration: A synthesis of science and management. Washington, DC: Island Press.

96. Osgood DT, Yozzo DJ, Chambers RM, Jacobson D, Hoffman T, et al. (2003) Tidal hydrology and habitat utilization by resident nekton in Phragmites and nonPhragmites marshes. Estuaries 26(2B): 522-33.

97. Hunter KL, Fox DA, Brown LM, Able KW (2006) Responses of resident marsh fishes to stages of Phragmites australis invasion in three mid Atlantic estuaries. Estuaries Coast 29(3): 487-498.

98. West JM, Zedler JB (2000) Marsh-creek connectivity: fish use of a tidal salt marsh in southern California. Estuaries 23(5): 699-710.

99. Weinstein MP, Litvin SY, Guida VG (2010) Stable isotope and biochemical composition of white perch in a Phragmites dominated salt marsh and adjacent waters. Wetlands 30: 1181-1191.

100. Kneib RT (1987) Predation risk and use of intertidal habitats by young fishes and shrimp. Ecology 68: 379-86.

101. Rountree RA, Able KW (1992) Foraging habits, growth, and temporal patterns of salt-marsh creek habitat use by young-of-year summer flounder in New Jersey. Trans Am Fish Soc 121: 765-776.

102. Kneib RT (1997) The role of tidal marshes in the ecology of estuarine nekton. Oceanogr Mar Biol Annu Rev 35: 163-220.

103. Allen EA, Fell PE, Peck MA, Gieg JA, Guthke CR, Newkirk MD (1994) Gut contents of common mummichogs, Fundulus heteroclitus L., in a restored impounded marsh and in natural reference marshes. Estuaries 17(2): 462-471.

104. Moreno-Mateos D, Power ME, Comin FA, Yockteng R (2012) Structural and functional loss in restored wetland ecosystems. PLoS Biol 10(1), doi:10.1371/journal.pbio.1001247. 
TABLES: CHAPTER 3

Table 3-1. Characteristics of our New England study sites. ${ }^{a}$

\begin{tabular}{|c|c|c|c|c|c|c|c|c|c|c|c|c|c|c|c|c|c|}
\hline 言 & 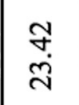 & $\begin{array}{l}\text { tr } \\
\text { à }\end{array}$ & 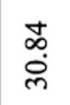 & 兽 & 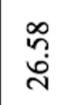 & $\frac{\hat{f}}{\dot{m}}$ & ஸિ. & $\underset{i}{\stackrel{p}{m}}$ & $\stackrel{\infty}{\stackrel{\infty}{\leftrightarrows}}$ & 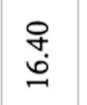 & $\stackrel{M}{\exists}$ & $\stackrel{\text { }}{\stackrel{\sim}{0}}$ & $\begin{array}{l}\stackrel{\infty}{\infty} \\
\stackrel{\sim}{\infty}\end{array}$ & 品 & 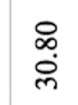 & 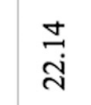 & 管鄫 \\
\hline 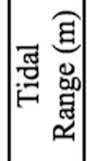 & 文 & $\begin{array}{l}\stackrel{\infty}{\infty} \\
0 \\
0\end{array}$ & fo & in & \begin{tabular}{|l}
$\infty$ \\
0 \\
0
\end{tabular} & 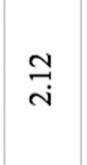 & के & $\begin{array}{l}2 \\
0 \\
0\end{array}$ & h̆ & ? & : & ల్రి & 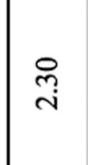 & $\stackrel{?}{i}$ & g & $\stackrel{\odot}{\underset{\sim}{\infty}}$ & 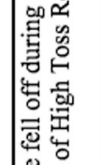 \\
\hline & $\exists$ & $\approx$ & $q$ & q & $\stackrel{\infty}{\sim}$ & $\stackrel{\infty}{\sim}$ & $\Rightarrow$ & 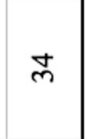 & $\stackrel{\dddot{\sigma}}{\sigma}$ & $\vec{m}$ & $\infty$ & లి & q & ते & ల్ల & \pm & 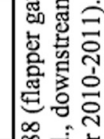 \\
\hline & 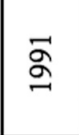 & \begin{tabular}{l}
$\infty$ \\
$\infty$ \\
$\vdots$ \\
\hdashline
\end{tabular} & Бे & बे & $\stackrel{\pi}{z}$ & घี & घ็ & घี & ส๊ & สึ & $\overrightarrow{\bar{N}}$ & ี๊ & घ็ & コ็ & $\Xi$ & $\stackrel{\pi}{\exists}$ & \\
\hline & 总 & 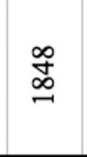 & 足 & 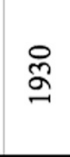 & $\Xi ّ$ & $\exists$ & घ็ & $\Xi$ & 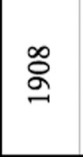 & 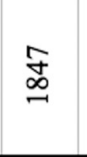 & 总 & 窟总 & घ็ & ส๊ & घี & ヨี & 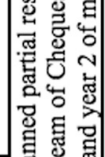 \\
\hline 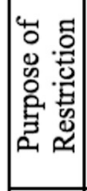 & 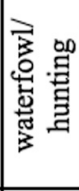 & $\begin{array}{l}\text { 总 } \\
\text { 莺 } \\
\text { 䙷 }\end{array}$ & 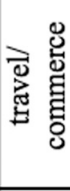 & 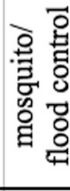 & త্ & घี & コ็ & छึ & 总 & 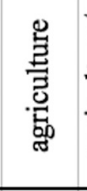 & 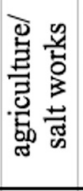 & $\begin{array}{l}\overrightarrow{\mathrm{o}} \\
\mathrm{i} \\
\mathrm{o} \\
\overrightarrow{\mathrm{g}} \\
\mathrm{g} \\
\end{array}$ & छ็ & घ็ & $\Xi$ & $\exists$ & \\
\hline & 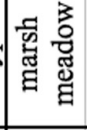 & 日 & ฐ & 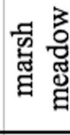 & 总悉 & 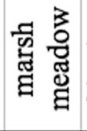 & 4 & 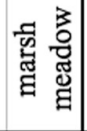 & 畓 & & ${ }^{2}$ & 总 & 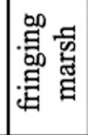 & 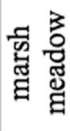 & & & \\
\hline . & $\stackrel{n}{a}$ & $\begin{array}{l}\tilde{y} \\
0\end{array}$ & $\stackrel{\varrho}{\rightrightarrows}$ & $\sum_{0}^{\tilde{L}}$ & $\stackrel{气}{G}$ & $\begin{array}{l}\tilde{y} \\
0 \\
0\end{array}$ & $\mathscr{G}$ & $\begin{array}{l}\Sigma \\
0 \\
0\end{array}$ & $\sum_{0}^{5}$ & $\stackrel{\mathscr{G}}{3}$ & 吾 & 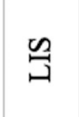 & 苛 & $\stackrel{\varrho}{\rightrightarrows}$ & 竞 & $\mathscr{a}$ & \\
\hline कै & 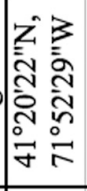 & & & & 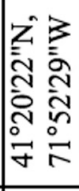 & & & 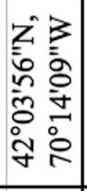 & 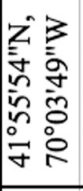 & 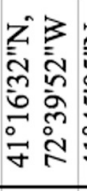 & & & 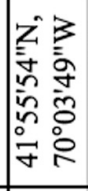 & $r$ & & 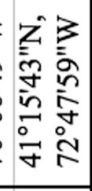 & 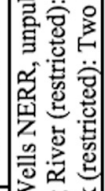 \\
\hline 荡 & 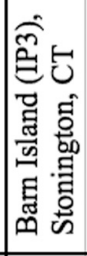 & 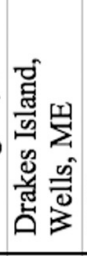 & & 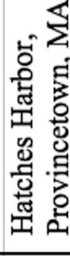 & 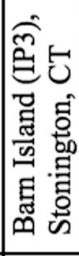 & 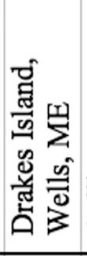 & 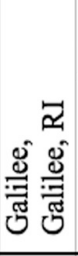 & 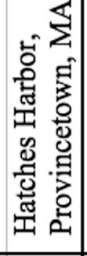 & 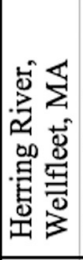 & 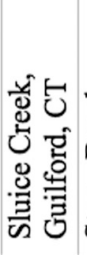 & 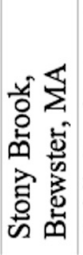 & 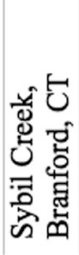 & 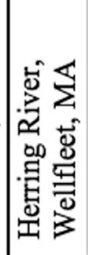 & & & 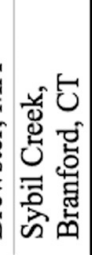 & 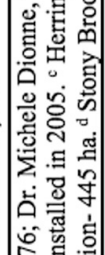 \\
\hline 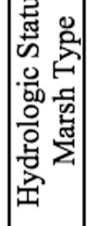 & 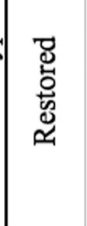 & $\begin{array}{l}\text { 芯 } \\
\text { 岁 } \\
\text { 品 }\end{array}$ & 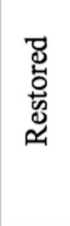 & 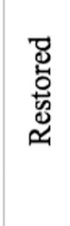 & 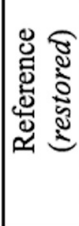 & 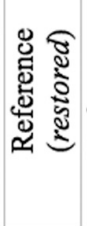 & 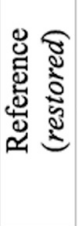 & 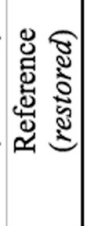 & 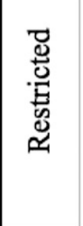 & 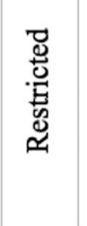 & 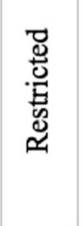 & 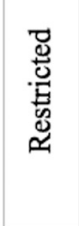 & 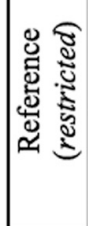 & 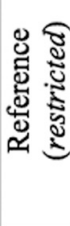 & 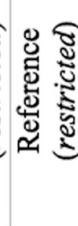 & 跣 & 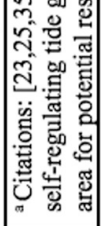 \\
\hline
\end{tabular}


Table 3-2. Mean water quality 2010-2011, by marsh type (standard deviations in parentheses; data pooled across regions and seasons).

\begin{tabular}{|l|c|c|c|c|}
\hline Response & Salinity (ppt) & Temperature $\left({ }^{\circ} \mathrm{C}\right)$ & Dissolved Oxygen $(\mathrm{mg} / \mathrm{L})$ & $\mathrm{n}$ \\
\hline Restored & $28.62(6.79)$ & $21.44(3.62)$ & $6.98(2.78)$ & 42 \\
\hline $\begin{array}{l}\text { Reference } \\
\text { (restored })\end{array}$ & $29.89(3.65)$ & $20.41(3.25)$ & $7.15(2.34)$ & 41 \\
\hline Restricted & $14.19(9.65)$ & $21.98(3.92)$ & $7.50(2.44)$ & 39 \\
\hline $\begin{array}{l}\text { Reference } \\
\text { restricted })\end{array}$ & $25.50(4.78)$ & $21.17(3.86)$ & $6.44(2.55)$ & 42 \\
\hline
\end{tabular}


Table 3-3. Parasites infecting Fundulus heteroclitus by marsh type, 2010-2011.

\begin{tabular}{|r|c|c|c|}
\hline & Ectoparasites & Endoparasites & Total \\
\hline Restored Abundance & 68 & 29 & 97 \\
\hline Total Infected & 56 & 18 & 72 \\
\hline Infection Intensity & 1.21 & 1.61 & 1.35 \\
\hline Prevalence & $7.46 \%$ & $2.40 \%$ & $9.59 \%$ \\
\hline Weighted Prevalence & $9.05 \%$ & $3.86 \%$ & $12.92 \%$ \\
\hline Reference (restored) & & & \\
\hline Abundance & 62 & 42 & 104 \\
\hline Total Infected & 53 & 13 & 62 \\
\hline Infection Intensity & 1.17 & 3.23 & 1.68 \\
\hline Prevalence & $7.02 \%$ & $1.72 \%$ & $8.21 \%$ \\
\hline Weighted Prevalence & $8.21 \%$ & $5.56 \%$ & $13.77 \%$ \\
\hline Abundance & & & \\
\hline Total Infected & 77 & 396 & 487 \\
\hline Infection Intensity & 1.18 & 3.00 & 185 \\
\hline Prevalence & $10.19 \%$ & $17.46 \%$ & 24.63 \\
\hline Weighted Prevalence & $12.04 \%$ & $52.38 \%$ & $64.42 \%$ \\
\hline Reference (restricted) & & & \\
\hline Abundance & 83 & 195 & 278 \\
\hline Total Infected & 69 & 70 & 125 \\
\hline Infection Intensity & 1.20 & 2.79 & 2.22 \\
\hline Prevalence & $9.15 \%$ & $9.28 \%$ & $16.58 \%$ \\
\hline Weighted Prevalence & $11.01 \%$ & $25.86 \%$ & $36.87 \%$ \\
\hline
\end{tabular}


Table 3-4. Mean proximate body composition of fish in study, 2010-2011 (standard deviations in parentheses; data by marsh type are pooled across regions and seasons; data by region, time, and sex are pooled across marsh types; reference marshes adjacent to the restored and restricted marshes are noted in parentheses).

\begin{tabular}{|l|c|c|c|c|c|c|c|}
\hline Response & $\begin{array}{c}\text { Lipid } \\
(\% \\
\text { dry })\end{array}$ & $\begin{array}{c}\text { Total } \\
\text { lipid } \\
(\mathrm{g})\end{array}$ & $\begin{array}{c}\text { Lean } \\
\text { mass } \\
(\% \text { dry })\end{array}$ & $\begin{array}{c}\text { Total } \\
\text { lean mass } \\
(\mathrm{g})\end{array}$ & $\begin{array}{c}\text { Water } \\
(\% \\
\text { wet })\end{array}$ & $\begin{array}{c}\text { Total } \\
\text { water } \\
(\mathrm{g})\end{array}$ & $\begin{array}{c}\text { Fish } \\
\text { length } \\
(\mathrm{mm})\end{array}$ \\
\hline Restored & 8.78 & 0.08 & 91.22 & 0.84 & 80.14 & 3.62 & 69.7 \\
& $(2.69)$ & $(0.05)$ & $(2.69)$ & $(0.38)$ & $(1.66)$ & $(1.50)$ & $(9.5)$ \\
\hline Reference & 9.09 & 0.06 & 90.91 & 0.63 & 80.56 & 2.81 & 63.6 \\
(restored $)$ & $(2.63)$ & $(0.04)$ & $(2.63)$ & $(0.32)$ & $(1.62)$ & $(1.38)$ & $(9.4)$ \\
\hline Restricted & 7.48 & 0.06 & 92.52 & 0.75 & 80.54 & 3.26 & 67.0 \\
& $(2.61)$ & $(0.04)$ & $(2.61)$ & $(0.32)$ & $(1.42)$ & $(1.29)$ & $(8.7)$ \\
\hline Reference & 8.62 & 0.10 & 91.38 & 0.96 & 80.04 & 4.59 & 71.6 \\
(restricted $)$ & $(2.49)$ & $(0.07)$ & $(2.49)$ & $(0.46)$ & $(1.75)$ & $(5.21)$ & $(10.0)$ \\
\hline Gulf of & 9.90 & 0.10 & 90.10 & 0.92 & 80.20 & 4.23 & 71.2 \\
Maine & $(2.20)$ & $(0.06)$ & $(2.20)$ & $(0.44)$ & $(1.58)$ & $(3.89)$ & $(10.4)$ \\
\hline Long & 7.08 & 0.05 & 92.92 & 0.67 & 80.44 & 2.90 & 64.7 \\
Island & $(2.33)$ & $(0.02)$ & $(2.33)$ & $(0.29)$ & $(1.67)$ & $(1.13)$ & $(8.0)$ \\
Sound & & & & & & & \\
\hline Summer & 7.51 & 0.06 & 92.49 & 0.76 & 81.71 & 3.63 & 69.9 \\
2010 & $(2.22)$ & $(0.04)$ & $(2.22)$ & $(0.28)$ & $(1.54)$ & $(1.25)$ & $(7.1)$ \\
\hline Fall 2010 & 8.41 & 0.08 & 91.59 & 0.85 & 80.05 & 3.60 & 69.7 \\
& $(2.50)$ & $(0.06)$ & $(2.50)$ & $(0.44)$ & $(1.51)$ & $(1.69)$ & $(10.7)$ \\
\hline Summer & 7.72 & 0.07 & 92.28 & 0.86 & 80.30 & 3.72 & 69.0 \\
2011 & $(2.25)$ & $(0.05)$ & $(2.25)$ & $(0.41)$ & $(1.10)$ & $(1.67)$ & $(9.4)$ \\
\hline Fall 2011 & 10.31 & 0.08 & 89.69 & 0.71 & 79.24 & 3.33 & 63.3 \\
& $(2.74)$ & $(0.06)$ & $(2.74)$ & $(0.41)$ & $(1.28)$ & $(5.24)$ & $(10.3)$ \\
\hline Males & 8.23 & 0.07 & 91.77 & 0.75 & 80.18 & 3.24 & 66.9 \\
& $(2.87)$ & $(0.05)$ & $(2.87)$ & $(0.38)$ & $(1.76)$ & $(1.46)$ & $(9.5)$ \\
\hline Females & 8.75 & 0.08 & 91.25 & 0.83 & 80.46 & 3.89 & 69.0 \\
& $(2.42)$ & $(0.05)$ & $(2.42)$ & $(0.41)$ & $(1.48)$ & $(3.86)$ & $(10.1)$ \\
\hline
\end{tabular}


Table 3-5. Results of repeated measures ANOVA for the restricted vs. reference systems [Model terms: Marsh type (termed "Marsh": comparison of restricted vs. reference); Time (comparison of the two marsh types within summer 2010, fall 2010, summer 2011, fall 2011); Region (comparison of the two marsh types within the Gulf of Maine vs. Long Island Sound)].

\begin{tabular}{|r|c|c|c|c|c|c|c|}
\hline & \multicolumn{2}{|c|}{ \% Lipid } & \multicolumn{2}{c|}{ \% Lean Dry Mass } & \multicolumn{2}{c|}{ \% Water } & \\
\hline Model Terms & Sign. & $\mathrm{t}$ & Sign. & $\mathrm{t}$ & Sign. & $\mathrm{t}$ & $\mathrm{d} . \mathrm{f}$. \\
\hline Marsh & $\mathrm{p}=0.0013$ & 3.45 & $\mathrm{p}=0.0013$ & -3.45 & $\mathrm{p}=0.5213$ & -0.65 & 40 \\
\hline Marsh x Region & & & & & & & \\
\hline LIS & $\mathrm{p}=0.0116$ & 2.65 & $\mathrm{p}=0.0116$ & -2.65 & $\mathrm{p}=0.3746$ & -0.90 & 40 \\
\hline Marsh x Time & & & & & & & \\
\hline Summer 2010 & $\mathrm{p}=0.0519$ & 1.96 & $\mathrm{p}=0.0519$ & -1.96 & $\mathrm{p}=0.4474$ & 0.76 & 120 \\
\hline Fall 2010 & $\mathrm{p}=0.0112$ & 2.58 & $\mathrm{p}=0.0112$ & -2.58 & $\mathrm{p}=0.3111$ & -1.02 & 120 \\
\hline Summer 2011 & $\mathrm{p}=0.0141$ & 2.49 & $\mathrm{p}=0.0141$ & -2.49 & $\mathrm{p}=0.3092$ & -1.02 & 120 \\
\hline Fall 2011 & $\mathrm{p}=0.1970$ & 1.30 & $\mathrm{p}=0.1970$ & -1.30 & $\mathrm{p}=0.5632$ & -0.58 & 120 \\
\hline Marsh x Sex & & & & & & & \\
\hline Fales & $\mathrm{p}=0.0068$ & 2.85 & $\mathrm{p}=0.0068$ & -2.85 & $\mathrm{p}=0.1892$ & -1.34 & 40 \\
\hline $\begin{array}{r}\text { Marsh x Region x } \\
\text { Sex }\end{array}$ & $\mathrm{p}=0.0027$ & 3.20 & $\mathrm{p}=0.0027$ & -3.20 & $\mathrm{p}=0.7592$ & 0.31 & 40 \\
\hline GOM, Males & $\mathrm{p}=0.0096$ & 2.72 & $\mathrm{p}=0.0096$ & -2.72 & $\mathrm{p}=0.0400$ & -2.12 & 40 \\
\hline GOM, Females & $\mathrm{p}=0.0801$ & 1.80 & $\mathrm{p}=0.0801$ & -1.80 & $\mathrm{p}=0.4887$ & 0.70 & 40 \\
\hline LIS, Males & $\mathrm{p}=0.1964$ & 1.31 & $\mathrm{p}=0.1964$ & -1.31 & $\mathrm{p}=0.8144$ & 0.24 & 40 \\
\hline LIS, Females & $\mathrm{p}=0.0088$ & 2.75 & $\mathrm{p}=0.0088$ & -2.75 & $\mathrm{p}=0.7863$ & -0.27 & 40 \\
\hline
\end{tabular}


Table 3-6. Mean otolith measurements for fish in study, 2010-2011 (standard deviations in parentheses; data by marsh type are pooled across regions and seasons; data for region, time, and sex are pooled across marsh types; reference marshes adjacent to the restored and restricted marshes are noted in parentheses).

\begin{tabular}{|l|c|c|c|c|c|c|}
\hline Response & $\begin{array}{c}\text { Daily } \\
\text { Growth } \\
(\mu \mathrm{m})\end{array}$ & $\begin{array}{c}\text { Otolith } \\
\text { Radius } \\
(\mu \mathrm{m})\end{array}$ & $\begin{array}{c}\text { Otolith } \\
\text { Length } \\
(\mu \mathrm{m})\end{array}$ & $\begin{array}{c}\text { Otolith } \\
\text { Height } \\
(\mu \mathrm{m})\end{array}$ & $\begin{array}{c}\text { Fish } \\
\text { Length } \\
(\mathrm{mm})\end{array}$ & $\begin{array}{c}\text { Fish Wet } \\
\text { Weight } \\
(\mathrm{g})\end{array}$ \\
\hline Restored & 2.16 & 719.16 & $\begin{array}{c}1496.63 \\
(0.66)\end{array}$ & $\begin{array}{c}1351.16 \\
(102.21)\end{array}$ & $\begin{array}{c}66.2 \\
(207.74)\end{array}$ & $\begin{array}{c}(157.28) \\
(11.9)\end{array}$ \\
\hline Reference & 2.26 & 669.96 & 1393.41 & 1271.54 & 61.7 & 3.09 \\
$($ restored $)$ & $(0.74)$ & $(99.81)$ & $(212.99)$ & $(167.68)$ & $(11.5)$ & $(1.84)$ \\
\hline Restricted & 2.21 & 692.70 & 1450.01 & 1324.26 & 61.2 & 2.98 \\
& $(0.79)$ & $(86.86)$ & $(193.28)$ & $(134.89)$ & $(11.1)$ & $(1.78)$ \\
\hline Reference & 2.26 & 726.75 & 1559.24 & 1391.76 & 67.8 & 4.29 \\
$($ restricted $)$ & $(0.75)$ & $(107.94)$ & $(227.14)$ & $(158.14)$ & $(12.7)$ & $(2.79)$ \\
\hline Gulf of Maine & 2.20 & 681.37 & 1451.10 & 1317.49 & 67.0 & 4.05 \\
& $(0.64)$ & $(99.97)$ & $(229.76)$ & $(172.15)$ & $(13.0)$ & $(2.59)$ \\
\hline Long Island & 2.24 & 721.39 & 1495.87 & 1348.28 & 62.0 & 3.18 \\
Sound & $(0.81)$ & $(102.13)$ & $(209.91)$ & $(153.15)$ & $(10.7)$ & $(1.81)$ \\
\hline Summer 2010 & 3.03 & 720.36 & 1501.03 & 1355.08 & 67.7 & 4.06 \\
& $(0.64)$ & $(92.25)$ & $(188.93)$ & $(134.94)$ & $(10.0)$ & $(1.96)$ \\
\hline Fall 2010 & 2.09 & 719.18 & 1520.13 & 1362.19 & 66.0 & 3.91 \\
& $(0.37)$ & $(107.44)$ & $(250.34)$ & $(179.38)$ & $(13.5)$ & $(2.77)$ \\
\hline Summer 2011 & 2.39 & 728.15 & 1521.30 & 1380.89 & 67.6 & 3.77 \\
& $(0.54)$ & $(103.99)$ & $(209.17)$ & $(153.29)$ & $(10.6)$ & $(1.85)$ \\
\hline Fall 2011 & 1.53 & 655.91 & 1384.16 & 1261.84 & 58.4 & 2.84 \\
& $(0.19)$ & $(93.36)$ & $(202.22)$ & $(156.15)$ & $(11.3)$ & $(2.10)$ \\
\hline Males & 2.21 & 691.85 & 1461.51 & 1330.16 & 62.8 & 3.23 \\
& $(0.71)$ & $(96.71)$ & $(207.13)$ & $(157.45)$ & $(10.8)$ & $(1.72)$ \\
\hline Females & 2.23 & 712.53 & 1487.33 & 1336.65 & 66.1 & 3.99 \\
& $(0.75)$ & $(108.28)$ & $(233.71)$ & $(169.28)$ & $(13.1)$ & $(2.67)$ \\
\hline
\end{tabular}




\section{FIGURES: CHAPTER 3}

Figure 3-1. Map of study site locations in New England.

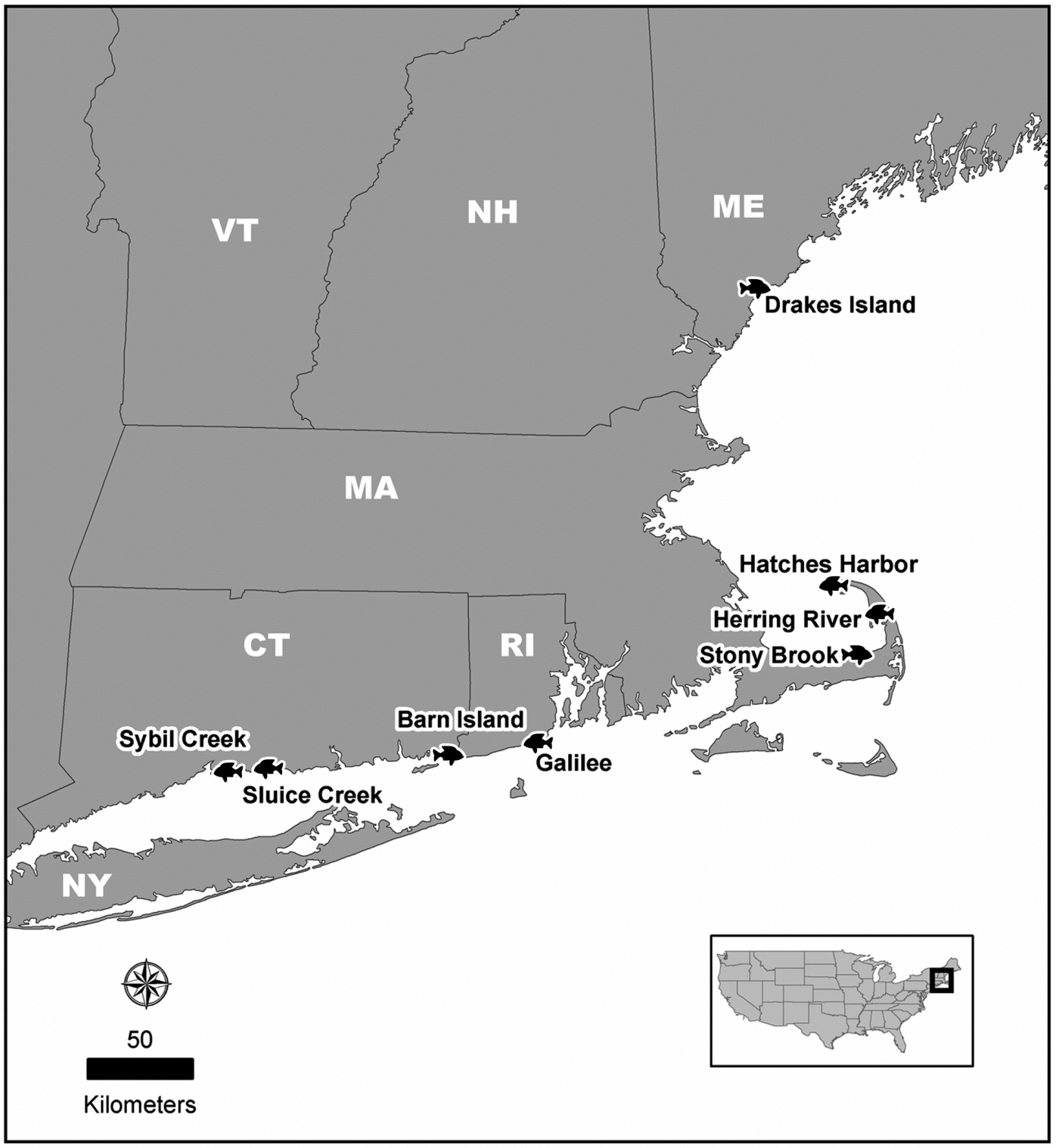


Figure 3-2. Proportion of fish parasitized (circles; females and males) or gravid (triangles; females only) by marsh type. Data is presented as the mean proportion \pm standard deviation.

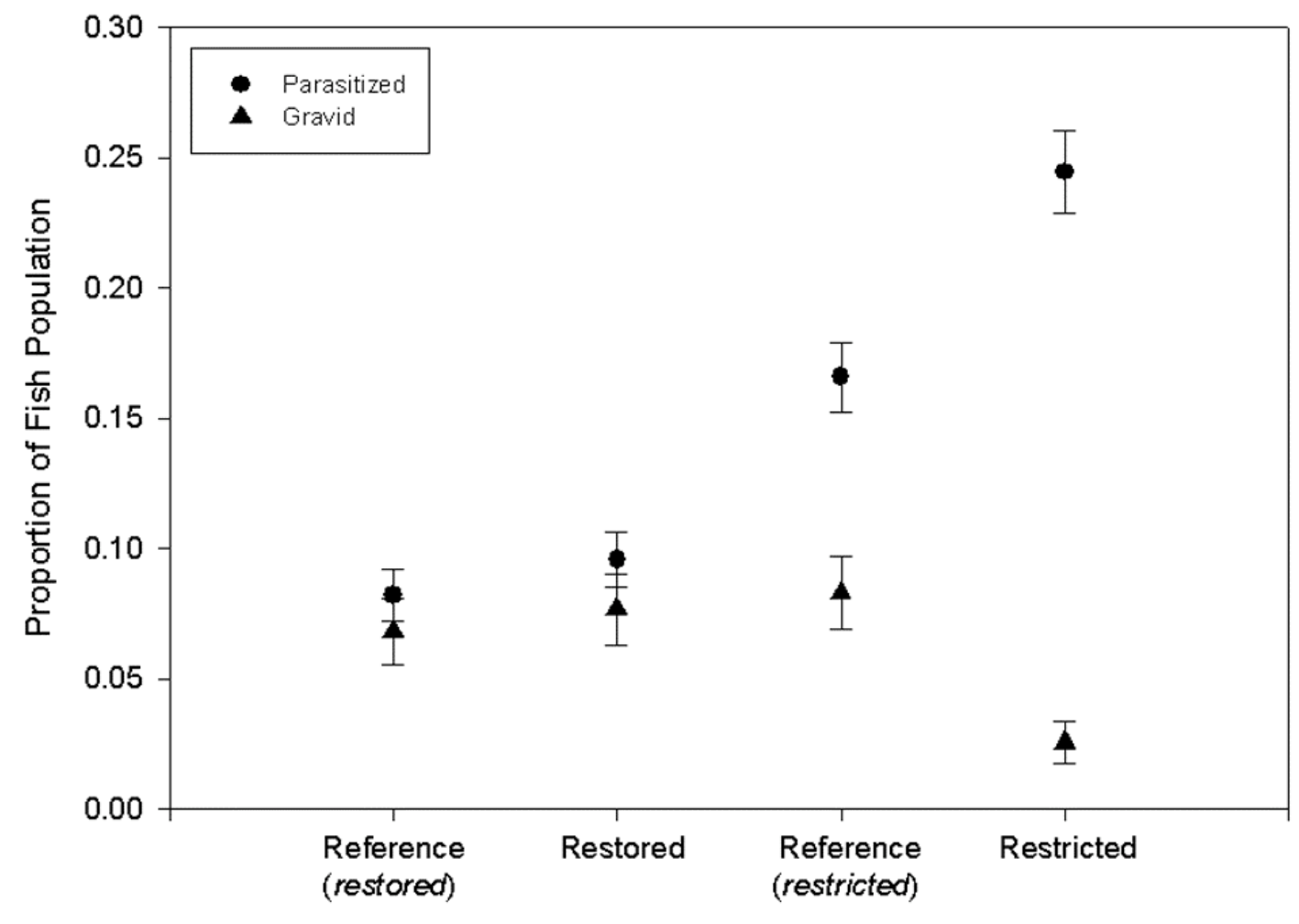


Figure 3-3. Proximate body composition of fish. Healthy fish only- data pooled across seasons, regions, and sex. Outlier circles represent the $5^{\text {th }}$ and $95^{\text {th }}$ percentiles and error bars the $10^{\text {th }}$ and $90^{\text {th }}$ percentiles for each population. (A) \% lipid mass (dry weight). (B) \% lean mass (dry weight). (C) \% water mass (wet weight).

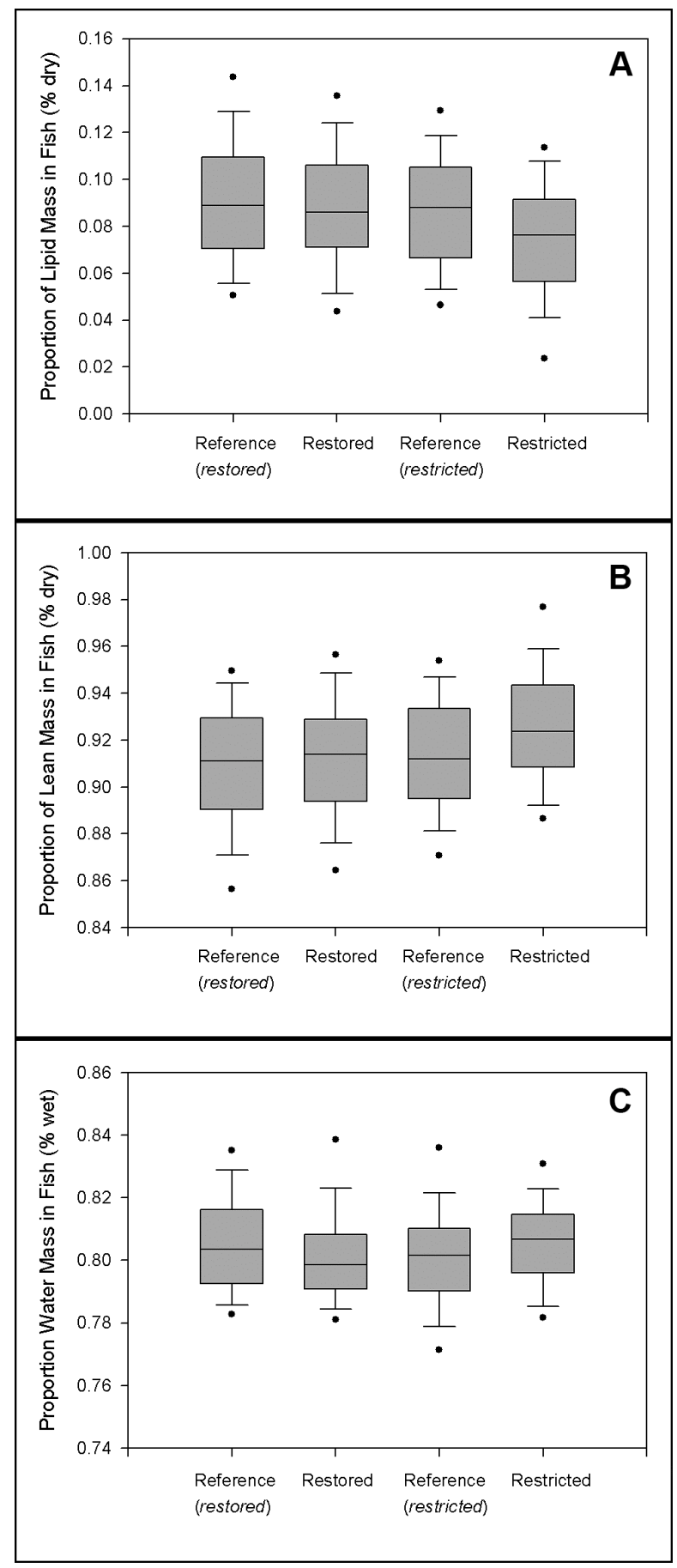


Figure 3-4. Number of fish captured by age group and marsh type.

\begin{tabular}{|r|r|r|r|r|r|}
\hline \multicolumn{1}{|c|}{} & & & & & \\
\hline
\end{tabular}


Figure 3-5. Fish length vs. otolith radius for healthy fish (Otolith radius $=-2.77341+$ $0.09572 *$ fish length; $\mathrm{p}<0.0001 ; \mathrm{r}^{2}=0.6628$ ).

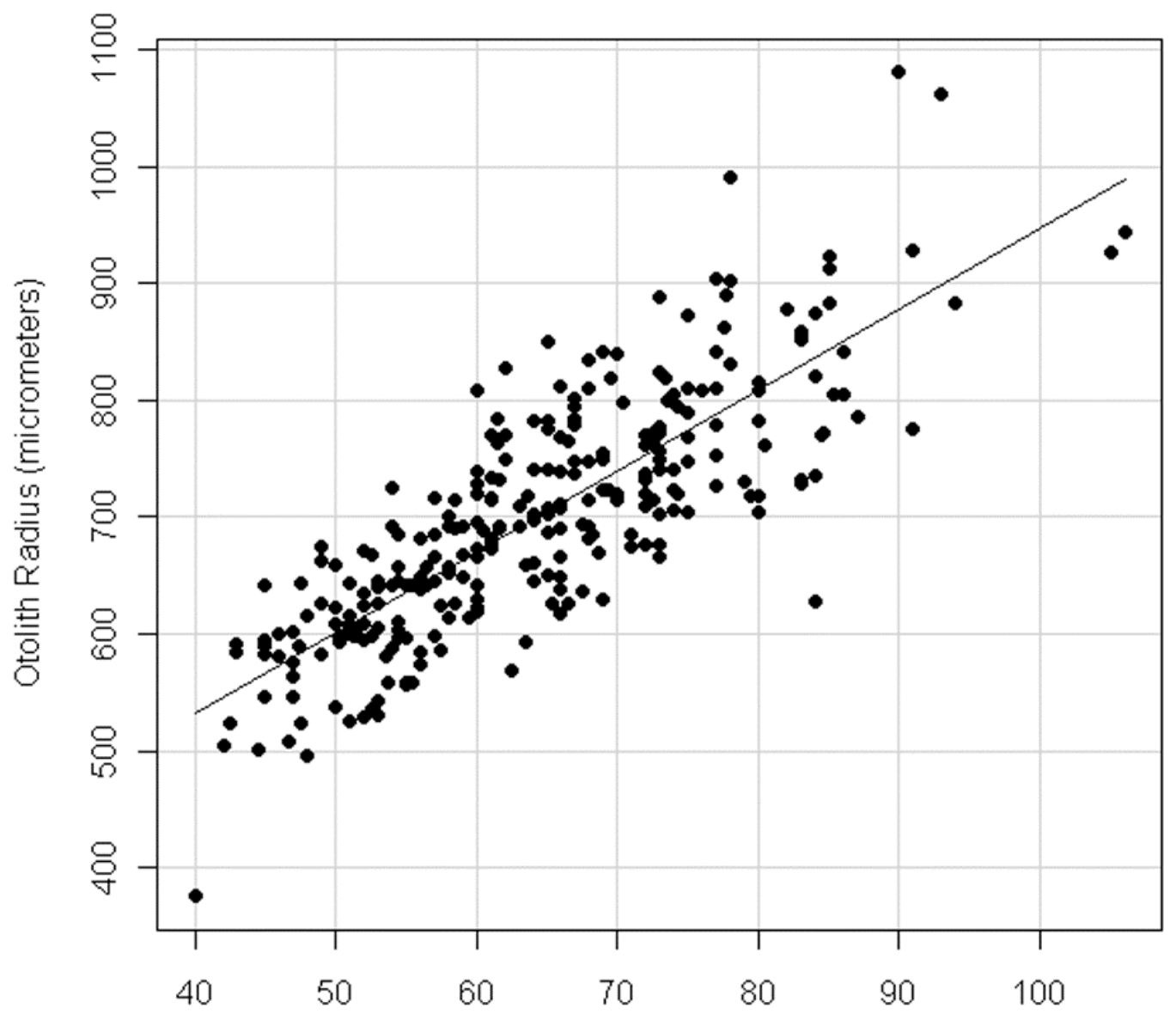

Fish Length (mm) 
Figure 3-6. Fish length vs. wet weight for healthy fish. Data pooled across seasons, regions, and by gender. (A) Restored vs. reference fish. (B) Restricted vs. reference fish.

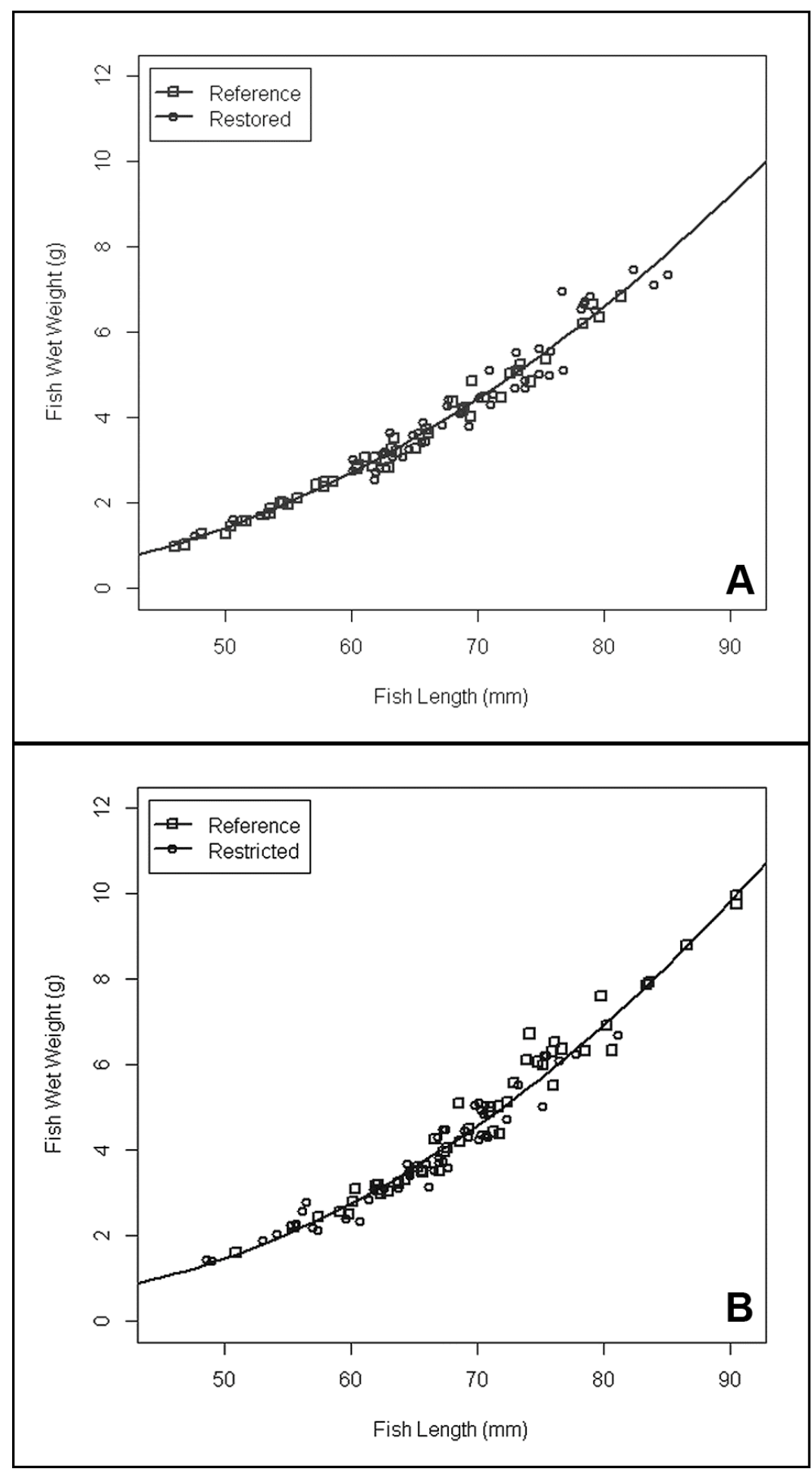




\section{CHAPTER 4}

\section{Tidal restriction may reduce female fish gravidity in salt marshes}

by

Kimberly L. Dibble ${ }^{1}$; Laura A. Meyerson ${ }^{1,2}$

is in revision at Marine Ecology Progress Series

${ }^{1}$ Department of Natural Resources Science, University of Rhode Island, 1 Greenhouse Road, Kingston, RI 02881.

${ }^{2}$ Institute of Botany, Department of Invasion Ecology, Academy of Sciences of the Czech Republic, Průhonice, CZ 252 43, Czech Republic. 


\begin{abstract}
Tidally restricted marshes often exhibit reductions in tidal range and marsh surface flooding that facilitate invasions by non-native plants such as Phragmites australis. Marsh accretion is common in P. australis- invaded systems due to high rhizome, root, and aboveground plant biomass, which can potentially affect spawning substrate available to fish at high tide. We examined populations of female Fundulus heteroclitus in New England salt marshes and found a significant reduction in the proportion of gravid fish in restricted marshes invaded by $P$. australis relative to adjacent unrestricted marshes, but no difference between restored and unrestricted marshes. Our results suggest that increased tidal flushing has restored the environmental conditions and habitat substrate necessary for F. heteroclitus reproduction. Our analyses also indicate that differences in gravidity are likely due to the habitat itself and not mean fish size (length, weight) or abiotic factors (temperature, dissolved oxygen). To expand on our research findings future studies should sample female $F$. heteroclitus over the progression of lunar periods from midMay to late July, include metrics of fecundity and spawning stock biomass, and determine whether fish are semilunar or continuous spawners at study sites.
\end{abstract}

Keywords: eggs; spawning; Phragmites australis; Fundulus heteroclitus; tidal restoration.

\title{
Introduction
}

Impoundments such as roads and bridges constructed across salt marshes reduce 
salinity, decrease tidal range, and cause marsh subsidence through the oxidation and decay of drained peat deposits (Portnoy and Giblin 1997). Combined with increased nitrogen inputs from coastal development, these factors have facilitated invasions by non-native plants including introduced Phragmites australis subsp. australis (common reed; hereafter introduced P. australis; Roman et al. 1984; Silliman and Bertness 2004). Over time, the reduction in tidal range associated with the restriction (Roman et al. 1984; Raposa and Roman 2003; Raposa 2008) combined with the dense belowground network of roots and rhizomes and high aboveground biomass mat of living and slowly decomposing $P$. australis organic matter can increase marsh surface elevation relative to mean high tide (Meyerson et al. 2000; Able et al. 2003; Rooth et al. 2003).

Current rates of marsh accretion due to $P$. australis colonization (3-4mm/year; Rooth et al. 2003) in combination with reduced marsh surface flooding frequency is reducing access to intertidal habitats used by fish and swimming crustaceans for feeding and refuge (Osgood et al. 2003; Hunter et al. 2006). Post-invasion changes in habitat structure may limit reproduction by hindering fish spawning and egg deposition on the marsh surface (Able and Hagan 2000; Balouskus and Targett 2012). In addition, the conversion of native marsh flora to introduced $P$. australis flattens marsh surface microtopography (Windham and Lathrop 1999) and decreases the availability of small water-filled depressions (Able et al. 2003) essential to larval and juvenile fish as nursery, feeding, and refuge habitats post-hatch (Able and Hagan 2000; Able et al. 2003; Hunter et al. 2006). Over the past three decades, re-establishing tidal flow into 
restricted New England salt marshes has proven to be an effective restoration strategy to eliminate or reduce $P$. australis cover, increase tidal range, and restore habitat function for nekton assemblages (Roman et al. 1984; James-Pirri et al. 2001; Warren et al. 2002; Raposa and Roman 2003; Chambers et al. 2012).

Reproduction of Fundulus heteroclitus (mummichogs), a small abundant marsh fish, occurs in intertidal and subtidal salt marsh habitats in April-June south of New Jersey (Able et al. 2007). In southern populations (Fundulus heteroclitus subsp. heteroclitus), spawning coincides with the high spring tides of the full and new moons (termed a "semilunar cycle") during which eggs are laid in high intertidal sites over a period of five or more days and then hatch two weeks later on the following spring tide (Taylor 1986; DiMichele and Westerman 1997; Nordlie 2006). For populations north of New Jersey (Fundulus heteroclitus subsp. macrolepidotus), semilunar spawning periodicity may be reduced or relaxed, during which eggs may be continuously present in the body cavity (Wallace and Selman 1980, 1981; Taylor 1986; Petersen et al. 2010) and spawning can occur daily on the highest tides during May-June. This could be a physiological adaptation to a shorter breeding season or reflect increased energy available for reproduction, which would relax the temporal selectivity of spawning observed in southern populations (Petersen et al. 2010).

Southern and northern populations of $F$. heteroclitus spawn on a variety of substrates near the high water mark in the intertidal zone, including decaying eelgrass/algae mats (Able and Hata 1984; Taylor 1986), bare gravel and mud associated with Spartina 
patens (Petersen et al. 2010), in sand (Taylor 1986), in Geukensia demissa mussel shells, or within the first axils of S. alterniflora stems (Taylor and DiMichele 1983; Taylor 1986). Aerial exposure of eggs is essential to survival, but eggs must be deposited in a moist, protected area immersed by lunar or semilunar tides to avoid desiccation and to trigger hatching. When suitable protective habitat (e.g., loose sand, Spartina stem axils, mussel shells) is not available, $F$. heteroclitus will deposit eggs in exposed sites in the intertidal zone (i.e., plant roots, debris, wrack; Taylor 1986), potentially exposing adults to predation by terrestrial predators during spawning. Invasion by $P$. australis may reduce marsh spawning habitat for $F$. heteroclitus in several ways. The greater stem height of introduced P. australis over Spartina spp. (Farnsworth and Meyerson 2003) potentially renders the leaves of the invader inaccessible for egg deposition. Introduced $P$. australis leaves are tightly held to the stem by persistent culm sheaths, a suboptimal habitat for egg deposition (Raichel et al. 2003). Fundulus heteroclitus will spawn on introduced $P$. australis stems but egg deposition only occurs in the hollows of broken dead culms that are submerged at high tide (Able and Hagan 2003) or if substrate is available adjacent to tidal creeks within P. australis stands (Raichel et al. 2003). Tidal creeks and rivulets are important access points for spawning that are often colonized during later invasion stages (Chambers et al. 1999; Able et al. 2003; Hunter et al. 2006), potentially making these habitats less accessible for reproduction and larval development (Able and Hagan 2000).

To date, two studies have provided evidence that $F$. heteroclitus are reproducing in $P$. australis invaded marshes in the mid-Atlantic (via the presence of eggs; Able and 
Hagan 2003; Raichel et al. 2003). However, to our knowledge, no study has examined female gravidity between unrestricted marshes that lack the invasive plant and restricted marshes colonized by $P$. australis. This research addresses the following questions: a) Is there a significant difference in the proportion of gravid females residing in tidally restricted vs. unrestricted marshes? b) Does gravidity differ between tidally restored and unrestricted marshes? and c) Are differences in the proportion of gravid females due to fish size or the habitat in which they reside?

\section{Methods}

Study Sites

We selected four tidally restricted (hereafter, "restricted") and four tidally restored ("restored") salt marshes invaded by introduced P. australis in New England (Fig. 41). We employed a matched pairs experimental design whereby each restricted or restored site was paired with an adjacent downstream, unrestricted ("reference") site that was sampled on the same day ( $\mathrm{n}=16$ marshes; 4 restricted, 4 restored, 8 reference). Based on previous studies, $F$. heteroclitus exhibit strong site fidelity and have limited movement between paired marshes (Fritz et al. 1975; Lotrich 1975; Skinner et al. 2005; Able et al. 2006; Eberhardt et al. 2011). Therefore, data from restricted marshes were only compared to data from the adjacent reference marshes, and data from restored marshes were only compared to data from adjacent reference marshes (i.e., four "marsh types" were analyzed; restricted/reference; restored/reference). Although our study design does not allow us to separate the effects of tidal restrictions from the effects of $P$. australis invasion, these two factors 
are often successive in New England salt marshes and both work to reduce tidal range and marsh surface access and hence, nekton support functions (Roman et al. 1984; Warren et al. 2002; Raposa and Roman 2003; Eberhardt et al. 2011).

The data presented herein is part of a larger multi-year research effort to biochemically quantify the impact of tidally restricted habitats on resident fish in comparison to unrestricted and restored marshes across a wide geographic area (Dibble and Meyerson, in press). Our related study was designed to avoid the $F$. heteroclitus spawning cycle; however, in 2011 fish exhibited delayed spawning in the Northeast as evidenced by the presence of ovulated, ripe eggs in female abdominal cavities. Spawning delays could have resulted from below seasonally cold air temperatures in winter associated with a strong negative phase of the Arctic Oscillation (NCDC 2011), record-breaking levels of precipitation consistent with spring La Niña patterns (NCDC 2011) that increased freshwater input and runoff into coastal systems, and/or cold anomalies of spring sea surface temperature in May in Long Island Sound and off Cape Cod (NEFSC 2011). We subsequently captured large quantities of gravid fish, providing an opportunity to assess reproduction in our study system. Therefore, these data represent one sampling period during one reproductive season for this fish species. We sampled all sites from July 11-23, 2011 along a south to north transect (Fig. 4-1) corresponding to the days leading up to full moon (on 7/15/11) for fish at our southern sites (sampled 7/11,7/12,7/14,7/15). We sampled northern sites as the full moon waned (7/18,7/19,7/20,7/23; new moon on 7/30). 


\section{Field and Laboratory Data}

Our study sites are located within the northern population range of $F$. heteroclitus subsp. macrolepidotus (Morin and Able 1983; Able and Felley 1986; Powers et al. 1986; Marteinsdottir and Able 1988, 1992) but we refer to our samples as $F$. heteroclitus throughout this manuscript. In July 2011 we collected $F$. heteroclitus from three randomly selected stations along the main tidal creek of each site $(n=48$ experimental units). At each station we deployed two minnow traps, combined the contents from both, and then randomly selected eight female fish representing the largest, smallest, and middle size ranges of fish available. We measured (fork length, $\mathrm{mm}$ ) weighed (nearest centigram), and humanely euthanized fish in the field using IACUC sanctioned procedures (protocol \#AN09-05-020). In addition to collecting fish samples, we measured salinity (ppt), temperature $\left({ }^{\circ} \mathrm{C}\right)$, and dissolved oxygen $(\mathrm{mg} / \mathrm{L})$ with a YSI Pro-2030 at every station on each sampling date. These were spot measurements recorded from approximately mid-way through flood tide to peak high tide (prior to ebbing) when fish were removed from the water column.

In the field and in the laboratory we noted that many gravid females extruded eggs from the body cavity with slight pressure to the abdomen. In the laboratory we used a scalpel and scissors to open the abdominal cavities of female fish and examined eggs (if present) under high light conditions. For our analysis, we used egg presence/ absence rather than specific metrics of fecundity (e.g., quantity of eggs) to assess fish gravidity. We only counted a fish as "gravid" if the fish was in the spawning phase of egg development, with transparent, ovulated eggs filling the body cavity. 


\section{Statistical Analysis}

We conducted all analyses using the SAS (v. 9.2) and R (v. 2.14.1) statistical software packages and developed figures using SigmaPlot (v. 9.0). July 2011 water quality data met assumptions of normality and we therefore compared marsh types using Analysis of Variance followed by Fisher's Protected Least Significant Difference tests. We conducted separate ANOVA's for each of the salinity, temperature, and dissolved oxygen variables.

To determine whether the tidally restricted and restored marshes harbored fewer gravid $F$. heteroclitus in comparison to those in reference marshes, we examined each of our hypotheses with a test of two proportions using the Normal approximation to the Binomial distribution. Our data met the approximation and hypothesis test assumptions, so we proceeded to compare the proportion of gravid females between restricted vs. reference marshes and the restored vs. reference marshes. Since the probability of female gravidity was small in comparison to sample size in the restricted marshes $(5 \leq(n \times \pi) \leq 20)$, we conducted a continuity correction to increase the quality of the normal approximation for the restricted vs. reference comparison (Ott and Longnecker 2010).

Because we captured a greater number of smaller fish in tidally restricted marshes in July 2011, we sought to determine whether differences in gravidity between marsh systems were due to fish size rather than the habitat itself. To examine our hypotheses, we used a logistic regression model with egg presence/absence as the response and 
included regressors for marsh type, fish length (covariate), and the interaction between marsh type and fish length. We conducted a second logistic regression using fish wet mass instead of length as the covariate. We used fish in reference marshes (adjacent to restored marshes) as our baseline group in both logistic regressions because they represent the samples least affected by tidal restrictions. Logistic regression has been used to model the relationship between a binary response and a continuous variable in other ecological studies (Morgan 2004; Eisenbies et al. 2007) and is therefore appropriate for this analysis.

\section{Results}

\section{Water Quality}

Water quality variables such as temperature can influence spawning, so we tested whether we could detect differences in salinity, temperature, and dissolved oxygen between the restricted vs. reference and restored vs. reference marsh types using data from July 2011. As expected, restricted marshes exhibited significant reductions in salinity relative to reference marshes $\left(\mathrm{p}<0.0001 ; \mathrm{t}_{44}=7.86\right.$; Table $\left.4-1\right)$. Salinity in restored vs. reference marshes was equivalent $\left(\mathrm{p}=1.000 ; \mathrm{t}_{44}=0.00\right)$. There was no significant difference in temperature between the reference vs. restricted marshes $\left(\mathrm{p}=0.0592 ; \mathrm{t}_{44}=-1.94\right)$, but there was a significant difference in temperature between the reference and restored marsh sites $\left(\mathrm{p}=0.0101 ; \mathrm{t}_{44}=-2.69\right)$. Dissolved oxygen was equivalent between both marsh comparisons (restored vs. reference: $\mathrm{p}=0.2085$, $\mathrm{t}_{44}=1.28$; restricted vs. reference: $\mathrm{p}=0.9238, \mathrm{t}_{44}=0.10$; Table $\left.4-1\right)$. 


\section{Proportion of Gravid Fish}

In total, we captured 389 female $F$. heteroclitus in July 2011, 85 of which were gravid (21.9\%). We found a significantly lower proportion of gravid F. heteroclitus inhabiting restricted marshes vs. reference marshes $\left(\mathrm{p}=0.0047 ; \chi^{2}{ }_{1}=8.01 ;\right.$ Fig. $\left.4-2\right)$. In restricted marshes, only 9 of 98 female fish were gravid (9.2\%), whereas in the reference marshes, 25 of 98 (25.5\%) fish were gravid. We found no significant difference in the proportion of gravid females residing in restored vs. reference marshes $\left(p=0.5358 ; \chi^{2}=0.39 ;\right.$ Fig. 4-2). In restored marshes, 27 of 95 females were gravid (28.4\%), while in the reference marshes 24 of 98 fish were gravid (24.5\%).

\section{Relationship Between Gravidity and Fish Size}

Larger, more mature female fish are more likely to be gravid than smaller and/or immature fish, so we examined the influence of fish size on reproductive capacity. Using logistic regression, we found a significant relationship between fish length and egg presence in our baseline group, reference (restored) $\left(p=0.0115 ; \chi^{2}=6.38\right)$, but not for the restored $\left(p=0.1603 ; \chi^{2}{ }_{1}=1.97\right)$, reference $($ restricted $)\left(p=0.8794 ; \chi^{2}=0.02\right)$, and restricted marsh types $\left(p=0.5340 ; \chi^{2}=0.39\right.$; Tables $\left.4-2,4-3\right)$. We also found a significant relationship between fish wet mass and egg presence in our baseline group, reference (restored) $\left(\mathrm{p}=0.0047 ; \chi^{2}{ }_{1}=8.00\right)$, but not for the restored $(\mathrm{p}=0.5309$; $\left.\chi_{1}^{2}=0.39\right)$, reference (restricted) $\left(p=0.4617 ; \chi^{2}{ }_{1}=0.54\right)$, and restricted marsh types $\left(p=0.9010 ; \chi^{2}=0.02 ;\right.$ Tables $\left.4-4,4-5\right)$. 


\section{Discussion}

Our results provide evidence that the proportion of gravid $F$. heteroclitus in restricted marshes may be reduced relative to fish in unrestricted marshes. Fish reproduction is influenced by multiple environmental, biological, and physiological cues including (but not limited to) lunar phase, water temperature, habitat availability, fish size and maturity, and environmental stressors that necessitate physiological trade-offs between growth, reproduction, and survival.

In this study we collected data on female fish gravidity due to anomalous seasonal conditions during the days leading up to and just past full moon. Most fish at our study sites are likely to be continuously gravid since they are from the northern population of $F$. heteroclitus macrolepidotus that can spawn daily regardless of the moon phase (Wallace and Selman 1980, 1981; Petersen et al. 2010). Fish from our southern sites in Connecticut are near the intergradation zone between subspecies (Able and Felley 1986) and may exhibit semilunar spawning; however, even if this is the case, we collected fish from those sites during the peak spawning period in the days leading up to full moon. Therefore, differences in the proportion of gravid females between marsh types sampled on the same day lends credence to our results and it is more likely that fish labeled as non-gravid may not have been reproducing in 2011.

Reproduction of $F$. heteroclitus is affected by water temperature and spawning habitat availability. Warmer water temperature behind tidal restrictions may increase the timing of spawning, which could have resulted in fewer gravid females at our 
restricted study sites. However, analysis of temperature data from July 2011 indicates a slightly warmer but non-significant difference in water temperature between the restricted and unrestricted sites. The difference between restored and unrestricted water temperature was significant, with higher temperatures measured behind the opened culvert/restriction. Therefore, concerns that increased water temperature has stimulated spawning is not valid for our study system; otherwise we would have seen reduced gravidity in the restored marsh fish as well.

While $F$. heteroclitus will deposit eggs in introduced $P$. australis stands (Able and Hagan 2003; Raichel et al. 2003), they do so only in areas adjacent to tidal creeks (Raichel et al. 2003), suggesting that marshes can maintain reproductive function if creeks remain open to tidal exchange. Changes in the depth, frequency, and extent of tidal flooding due to restrictions (Roman et al. 1984; Raposa and Roman 2003; Raposa 2008) coupled with microhabitat changes in spawning substrate due to the plant invader (Raichel et al. 2003) could reduce the suitability of spawning habitat for $F$. heteroclitus or the survival of larval and juvenile $F$. heteroclitus post-hatch (Able and Hagan 2000). Over the long term, fish isolated in restricted marshes due to impoundments (Eberhardt et al. 2011), combined with reduced reproduction and decreased offspring survival, could alter gene flow and potentially affect future population structure and genetic diversity of restricted $F$. heteroclitus.

Fish reproduction is also influenced by biological cues and by physiological tradeoffs. Longer, more massive, and hence older fish are more likely to invest resources into 
reproduction rather than growth or survival. The length and weight ranges of nongravid and gravid $F$. heteroclitus overlapped across all marsh types but restricted fish were slightly smaller than those in the other three marshes studied. Even so, our finding that fish length and weight influences gravidity was only true for the fish in the unrestricted marshes adjacent to restored sites and not in the other three marsh types, indicating that fish captured in restored, restricted, and reference marshes adjacent to restricted sites can produce eggs at a range of lengths and weights (60$118 \mathrm{~mm} ; 2.7-24.9 \mathrm{~g}$; Tables 4-2, 4-4).

Fish exhibit life-long tradeoffs between growth, reproduction, and survival (Jorgensen et al. 2006). Reductions in gravidity and fecundity occur when food consumption declines (Black et al. 1998) or when body condition is poor (Jorgensen et al. 2006) with fish forgoing ovulation and spawning while channeling energy to growth and survival. Reduced access to prey inhabiting the marsh surface can decrease weight gain and the growth rate of $F$. heteroclitus in comparison to those only having access to the creek bed (Weisberg and Lotrich 1982). Since fish differentially allocate finite resources to egg production, growth, and energy storage (Jorgensen et al. 2006), any reduction in access to the marsh surface may result in trade-offs detectable at the population level. While morphological indicators do not show a difference in length and weight of adult $F$. heteroclitus residing in invaded vs. uninvaded marshes, physiological indicators have shown reductions in energy reserves (Weinstein et al. 2009; Dibble and Meyerson, in press), which could result in trade-offs to reproduction in these resident marsh fish. 
Our results suggest that the proportion of gravid female fish is reduced in tidally restricted salt marshes relative to unrestricted habitats. Although our results are from samples taken during a single lunar period in a possible anomalous sampling year, we observed a similar pattern in the July 2010 field season, but did not include these data in this analysis due to small sample size. A broader study specifically designed to capture spawning in all lunar phases is needed to quantify reproductive output and spawning capacity in these salt marsh systems. Future research could be designed to assess the reproductive condition of sexually mature adults during peak spawning season (i.e., circulating sex steroids, gonatal size, hepatic size, yolk protein synthesis, etc.) in relation to total energy reserves, dietary consumption, spawning substrate, and environmental conditions in restricted and unrestricted marshes. Additional research could also investigate the end points of reproduction and development, including viability of eggs and sperm, embryo mortality, developmental defects, time to hatching, larval prey capture ability, startle response, neurotransmitter levels, genetic diversity, and many others (Burnett et al. 2007). Such studies would supplement existing knowledge of impacts to the larval, juvenile, and adult life history stages and provide a clearer picture of potential impacts to fish in tidally restricted marshes colonized by common reed.

\section{Acknowledgments}

We thank Penelope Pooler for her assistance with this analysis and for her review of this manuscript. Special thanks to Peter August and the University of Rhode Island (URI) Environmental Data Center for drafting the map of our study sites. The 
following agencies and organizations provided support: EPA STAR Graduate

Fellowship (FP-91710001-0), National Oceanic and Atmospheric Administration

NERR Graduate Fellowship (NA09NOS4200041), National Science Foundation

(NSF) IGERT Grant to the Coastal Institute at URI (0504103), US NSF DEB Award

(1049914), Philanthropic Educational Organization (Lellis-Dib3158688), Northeast

Aquatic Plant Management Society, Rhode Island Natural History Survey and The

Nature Conservancy of Rhode Island (Lellis-Dibble 05-30-09), URI Agricultural

Experiment Station (RI00H-332, 311000-6044), URI Coastal Fellows Program, and

the U.S. and Czech Fulbright Commissions.

\section{Literature Cited}

Able KW, Balletto JH, Hagan SM, Jivoff PR, Strait K (2007) Linkages between salt marshes and other nekton habitats in Delaware Bay, USA. Rev Fish Sci 15:161.

Able KW, Felley JD (1986) Geographical variation in Fundulus heteroclitus: tests for concordance between egg and adult morphologies. Am Zool 26(1):145-157.

Able KW, Hagan SM (2000) Effects of common reed (Phragmites australis) invasion on marsh surface macrofauna: Response of fishes and decapod crustaceans. Estuaries 23(5):633-646.

Able KW, Hagan SM (2003) Impact of Common Reed, Phragmites australis, on Essential Fish Habitat: Influence on Reproduction, Embryological Development, and Larval Abundance of Mummichog (Fundulus heteroclitus). Estuaries 26(1):40-50.

Able KW, Hagan SM, Brown SA (2006) Habitat use, movement, and growth of young-of-the-year Fundulus spp. in southern New Jersey salt marshes: comparisons based on tag/recapture. J Exp Mar Biol Ecol 335:177-187.

Able KW, Hagan SM, Brown SA (2003) Mechanisms of Marsh Habitat Alteration Due to Phragmites: Response of Young-of-the-year Mummichog (Fundulus heteroclitus) to Treatment for Phragmites Removal. Estuaries 26(2B):484-494. 
Able KW, Hata D (1984) Reproductive behavior in the Fundulus heteroclitus-F. grandis complex. Copeia 1984(4):820-25.

Balouskus RG, Targett TE (2012) Egg deposition by Atlantic silverside, Menidia menidia: substrate utilization and comparison of natural and altered shoreline type. Estuaries Coast 35:1100-1109.

Black DE, Gutjahr-Gobell R, Pruell RJ, Bergen B, McElroy AE (1998) Effects of a mixture of non-ortho and mono-ortho-polychlorinated biphenyls on reproduction in Fundulus heteroclitus (Linnaeus). Environ Toxicol Chem 17(7):1396-1404.

Burnett KG, Bain LJ, Baldwin WS, Callard GV, Cohen S, Di Giulio RT, Evans DH, Gomez-Chiarri M, Hahn ME, Hoover CA, Karchner SI, Katah F, MacLatchy DL, Marshall WS, Meyer JN, Nacci DE, Oleksiak MF, Rees BB, Singer TD, Stegeman JJ, Towle DW, Van Veld PA, Vogelbein WK, Whitehead A, Winn RN, Crawford DL (2007) Fundulus as the premier teleost model in environmental biology: opportunities for new insights using genomics. Comp Biochem Physiol D 2:257-286.

Chambers RM, Meyerson LA, Dibble KL (2012) Ecology of Phragmites australis and responses to tidal restoration. In: Roman C, Burdick D (ed) Tidal marsh restoration: A synthesis of science and management. Island Press, Washington, DC.

Chambers RM, Meyerson LM, Saltonstall K (1999) Expansion of Phragmites australis into tidal wetlands of North America. Aquat Bot 64:261-73.

Dibble KL, Meyerson LA (in press) Tidal flushing restores the physiological condition of fish residing in degraded salt marshes. PLoS ONE.

DiMichele L, Westerman ME (1997) Geographic variation in development rate between populations of the teleost Fundulus heteroclitus. Mar Biol 128:1-7.

Eberhardt AL, Burdick DM, and Dionne M (2011) The effects of road culverts on nekton in New England salt marshes: Implications for tidal restoration. Rest Ecol 19(6):776-785.

Eisenbies MH, Davidson C, Johnson J, Amateis R, Gottschalk K (2007) Tree mortality in mixed pine-hardwood stands defoliated by the European gypsy moth (Lymantria dispar L.). Forest Sci 53(6):683-91.

Farnsworth EJ, Meyerson LA (2003) Comparative ecophysiology of four wetland plant species along a continuum of invasiveness. Wetlands 23(4):750-62. 
Fritz ES, Meredith WH, Lotrich VA (1975) Fall and winter movements and activity level of the mummichog, Fundulus heteroclitus, in a tidal creek. Chesap Sci 16(3):211-215.

Hunter KL, Fox DA, Brown LM, Able KW (2006) Responses of resident marsh fishes to stages of Phragmites australis invasion in three mid Atlantic estuaries. Estuaries Coast 29(3):487-498.

James-Pirri MJ, Raposa KB, Catena JG (2001) Diet composition of mummichogs, Fundulus heteroclitus, from restoring and unrestricted regions of a New England (U.S.A.) salt marsh. Estuar Coast Shelf S 53:205-213.

Jorgensen C, Ernande B, Fiksen O, Dieckmann U (2006) The logic of skipped spawning in fish. Can J Fish Aquat Sci 63:200-11.

Lotrich VA (1975) Summer home range and movements of Fundulus heteroclitus (Pisces: Cyprinodontidae) in a tidal creek. Ecology 56(1):191-198.

Marteinsdottir G, Able KW (1988) Geographic variation in egg size among populations of the mummichog, Fundulus heteroclitus (Pisces: Fundulidae). Copeia 1988(2):471-478.

Marteinsdottir G, Able KW (1992) Influence of egg size on embryos and larvae of Fundulus heteroclitus (L.). J Fish Biol 41:883-896.

Meyerson LA, Saltonstall K, Windham L, Kiviat E, Findlay S (2000) A comparison of Phragmites australis in freshwater and brackish marsh environments in North America. Wetl Ecol Mgmt 8:89-103.

Morgan MJ (2004) The relationship between fish condition and the probability of being mature in American plaice (Hippoglossoides platessoides). ICES J Mar Sci 61:64-70.

Morin RP, Able KW (1983) Patterns of geographic variation in the egg morphology of the Fundulid fish, Fundulus heteroclitus. Copeia 1983(3):726-740.

NOAA National Climatic Data Center (NCDC) (2011) State of the Climate: National Overview for Annual 2011. Accessed 21 August 2012. http://www.ncdc.noaa.gov/sotc/national/2011/13

NOAA Northeast Fishery Science Center (NEFSC) (2011) Ecosystem advisory for the Northeast Shelf Large Marine Ecosystem: Spring sea surface temperature distribution. Advisory 2011-No. 2. Accessed 21 August 2012. http://www.nefsc.noaa.gov/ecosys/advisory/fall2011/adv3.html 
Nordlie FG (2006) Physiochemical environments and tolerances of cyprinodontoid fishes found in estuaries and salt marshes of eastern North America. Rev Fish Biol Fisheries 16:51-106.

Osgood DT, Yozzo DJ, Chambers RM, Jacobson D, Hoffman T, et al. (2003) Tidal hydrology and habitat utilization by resident nekton in Phragmites and nonPhragmites marshes. Estuaries 26(2B):522-33.

Ott RL, Longnecker M (2010) An introduction to statistical methods and data analysis, $6^{\text {th }}$ edition. Brooks/Cole, Belmont, CA. pp. 191-194.

Petersen CW, Salinas S, Preston RL, and Kidder GW III (2010) Spawning periodicity and reproductive behavior of Fundulus heteroclitus in a New England salt marsh. Copeia 2010(2):203-10.

Portnoy JW, Giblin AE (1997) Effects of historic tidal restrictions on salt marsh sediment chemistry. Biogeochem 36:275-303.

Powers DA, Ropson I, Brown DC, Van Beneden R, Cashon R, Gonzalez-Villaseñor LI, DiMichele JA (1986) Genetic variation in Fundulus heteroclitus: geographic distribution. Am Zool 26(1):131-144.

Raichel DL, Able KW, Hartman JM (2003) The influence of Phragmites (common reed) on the distribution, abundance, and potential prey of a resident marsh fish in the Hackensack Meadowlands, New Jersey. Estuaries 26(2B):511-21.

Raposa KB (2008) Early ecological responses to hydrologic restoration of a tidal pond and salt marsh complex in Narragansett Bay, Rhode Island. J Coast Res 55:180-92.

Raposa KB, Roman CT (2003) Using gradients in tidal restriction to evaluate nekton community responses to salt marsh restoration. Estuaries 28(1):98-105.

Roman CT, Niering WA, Warren RS (1984) Salt marsh vegetation change in response to tidal restriction. Env Mgmt 8:141-150.

Rooth JE, Court Stevenson J, Cornwell JC (2003) Increased sediment accretion rates following invasion by Phragmites australis: The role of litter. Estuaries 26(2B):475-83.

Silliman BR, Bertness MD (2004) Shoreline development drives invasion of Phragmites australis and the loss of plant diversity on New England salt marshes. Conserv Biol 18(5):1424-34. 
Skinner MA, Courtenay SC, Parker WR, Curry RA (2005) Site fidelity of mummichogs (Fundulus heteroclitus) in an Atlantic Canadian estuary. Water Qual Res J Canada 40(3):288-298.

Taylor MH (1986) Environmental and endocrine influences on reproduction of Fundulus heteroclitus. Am Zool 26(1):159-171.

Taylor MH, DiMichele L (1983) Spawning site utilization in a Delaware population of Fundulus heteroclitus (Pisces: Cyprinodontidae). Copeia 1983(3):719-25.

Wallace RA, Selman K (1980) Oogenesis in Fundulus heteroclitus. II. The transition from vitellogenesis into maturation. Gen Comp Endocrinol 42(3):345-354.

Wallace RA, Selman K (1981) The reproductive activity of Fundulus heteroclitus females from Woods Hole, Massachusetts, as compared with more southern locations. Copeia 1981(1):212-215.

Warren RS, Fell PE, Rozsa R, Brawley AH, Orsted AC, et al. (2002) Salt marsh restoration in Connecticut: 20 years of science and management. Rest Ecol 10(3):497-513.

Weinstein MP, Litvin SY, and Guida VG (2009) Essential Fish Habitat and wetland restoration success: A tier III approach to the biochemical condition of common mummichog Fundulus heteroclitus in common reed Phragmites australis- and smooth cordgrass Spartina alterniflora- dominated salt marshes. Estuaries Coast 32:1011-1022.

Weisberg SB, Lotrich VA (1982) The importance of an infrequently flooded intertidal marsh surface as an energy source for the mummichog Fundulus heteroclitus: an experimental approach. Mar Biol 66:307-10.

Windham L, Lathrop RG Jr. (1999) Effects of Phragmites australis (Common Reed) invasion on aboveground biomass and soil properties in brackish tidal marsh of the Mullica River, New Jersey. Estuaries 22(4):927-935. 


\section{TABLES: CHAPTER 4}

Table 4-1. Mean water quality measurements (standard deviations in parentheses).

\begin{tabular}{|l|l|l|l|l|}
\hline Marsh Type & Salinity & Temperature & Dissolved Oxygen & $\mathrm{n}$ \\
\hline Reference (restricted) & $25.9(3.8)$ & $24.7(1.0)$ & $7.4(2.4)$ & 12 \\
\hline Restricted & $10.8(8.4)$ & $26.1(1.8)$ & $7.3(2.5)$ & 12 \\
\hline Reference (restored) & $30.9(1.3)$ & $20.3(2.9)$ & $7.4(2.0)$ & 12 \\
\hline Restored & $30.9(1.4)$ & $22.4(1.1)$ & $6.2(2.5)$ & 12 \\
\hline
\end{tabular}


Table 4-2. Mean fish length, range (in parentheses), and sample size of female $F$. heteroclitus.

\begin{tabular}{|l|l|l|}
\hline Marsh Type & Non-gravid & Gravid \\
\hline Reference (restricted) & $73.3 \mathrm{~mm}(43-103), \mathrm{n}=73$ & $82.7 \mathrm{~mm}(67-118), \mathrm{n}=25$ \\
\hline Restricted & $64.4 \mathrm{~mm}(50-95), \mathrm{n}=89$ & $73.9 \mathrm{~mm}(60-104), \mathrm{n}=9$ \\
\hline Reference (restored) & $69.1 \mathrm{~mm}(41-102), \mathrm{n}=74$ & $77.3 \mathrm{~mm}(63-103), \mathrm{n}=24$ \\
\hline Restored & $71.8 \mathrm{~mm}(42-101), \mathrm{n}=68$ & $84.4 \mathrm{~mm}(63-104), \mathrm{n}=27$ \\
\hline
\end{tabular}


Table 4-3. Logistic regression using fish length as a covariate [baseline group=reference $($ restored); $1=$ reference $($ restricted); $x 2=$ restored; $33=$ restricted; $\mathrm{x} 4=$ fish length].

\begin{tabular}{|c|c|c|c|c|c|c|c|}
\hline Parameter & d.f. & Estimate & $\begin{array}{c}\text { Standard } \\
\text { Error }\end{array}$ & \multicolumn{2}{|c|}{$\begin{array}{c}\text { Wald 95\% } \\
\text { Confidence Limits }\end{array}$} & $\begin{array}{c}\text { Wald Chi- } \\
\text { Square }\end{array}$ & p-value \\
\hline Intercept & 1 & -4.9699 & 1.5804 & -8.0675 & -1.8723 & 9.89 & 0.0017 \\
\hline $\mathrm{x} 1$ & 1 & -0.5083 & 2.2220 & -4.8633 & 3.8468 & 0.05 & 0.8191 \\
\hline $\mathrm{x} 2$ & 1 & -3.5463 & 2.5142 & -8.4740 & 1.3814 & 1.99 & 0.1584 \\
\hline $\mathrm{x} 3$ & 1 & -2.4488 & 2.6673 & -7.6767 & 2.7791 & 0.84 & 0.3586 \\
\hline $\mathrm{x} 4$ & 1 & 0.0525 & 0.0208 & 0.0118 & 0.0932 & 6.38 & 0.0115 \\
\hline $\mathrm{x} 1 \mathrm{x} 4$ & 1 & 0.0043 & 0.0285 & -0.0516 & 0.0602 & 0.02 & 0.8794 \\
\hline $\mathrm{x} 2 \mathrm{x} 4$ & 1 & 0.0448 & 0.0319 & -0.0177 & 0.1074 & 1.97 & 0.1603 \\
\hline $\mathrm{x} 3 \mathrm{x} 4$ & 1 & 0.0225 & 0.0362 & -0.0485 & 0.0935 & 0.39 & 0.5340 \\
\hline
\end{tabular}


Table 4-4. Mean fish wet mass, range (in parentheses), and sample size of female $F$.

heteroclitus.

\begin{tabular}{|l|l|l|}
\hline Marsh Type & Non-gravid & Gravid \\
\hline Reference (restricted) & $5.5 \mathrm{~g}(0.9-16.7), \mathrm{n}=73$ & $8.3 \mathrm{~g}(3.9-24.9), \mathrm{n}=25$ \\
\hline Restricted & $3.5 \mathrm{~g}(1.2-12.5), \mathrm{n}=89$ & $5.0 \mathrm{~g}(2.7-9.9), \mathrm{n}=9$ \\
\hline Reference (restored) & $4.6 \mathrm{~g}(0.6-15.3), \mathrm{n}=74$ & $6.7 \mathrm{~g}(3.3-14.9), \mathrm{n}=24$ \\
\hline Restored & $5.0 \mathrm{~g}(0.9-13.7), \mathrm{n}=68$ & $8.4 \mathrm{~g}(3.1-16.4), \mathrm{n}=27$ \\
\hline
\end{tabular}


Table 4-5. Logistic regression using fish wet mass as a covariate [baseline group=reference (restored); x1=reference (restricted); x2=restored; x3=restricted; $\mathrm{x} 4=$ fish wet mass)].

\begin{tabular}{|c|c|c|c|c|c|c|c|}
\hline Parameter & d.f. & Estimate & $\begin{array}{c}\text { Standard } \\
\text { Error }\end{array}$ & \multicolumn{2}{|c|}{$\begin{array}{c}\text { Wald 95\% } \\
\text { Confidence Limits }\end{array}$} & $\begin{array}{c}\text { Wald Chi- } \\
\text { Square }\end{array}$ & p-value \\
\hline Intercept & 1 & -2.5351 & 0.5798 & -3.6714 & -1.3988 & 19.12 & $<.0001$ \\
\hline $\mathrm{x} 1$ & 1 & 0.3269 & 0.7650 & -1.1725 & 1.8263 & 0.18 & 0.6692 \\
\hline $\mathrm{x} 2$ & 1 & -0.5297 & 0.8440 & -2.1840 & 1.1246 & 0.39 & 0.5303 \\
\hline $\mathrm{x} 3$ & 1 & -0.7195 & 0.8915 & -2.4668 & 1.0279 & 0.65 & 0.4197 \\
\hline $\mathrm{x} 4$ & 1 & 0.2544 & 0.0899 & 0.0781 & 0.4307 & 8.00 & 0.0047 \\
\hline $\mathrm{x} 1 \mathrm{x} 4$ & 1 & -0.0816 & 0.1109 & -0.2990 & 0.1357 & 0.54 & 0.4617 \\
\hline $\mathrm{x} 2 \mathrm{x} 4$ & 1 & 0.0765 & 0.1221 & -0.1628 & 0.3157 & 0.39 & 0.5309 \\
\hline $\mathrm{x} 3 \mathrm{x} 4$ & 1 & -0.0192 & 0.1542 & -0.3214 & 0.2831 & 0.02 & 0.9010 \\
\hline
\end{tabular}




\section{FIGURES: CHAPTER 4}

Figure 4-1. Study site locations in New England.

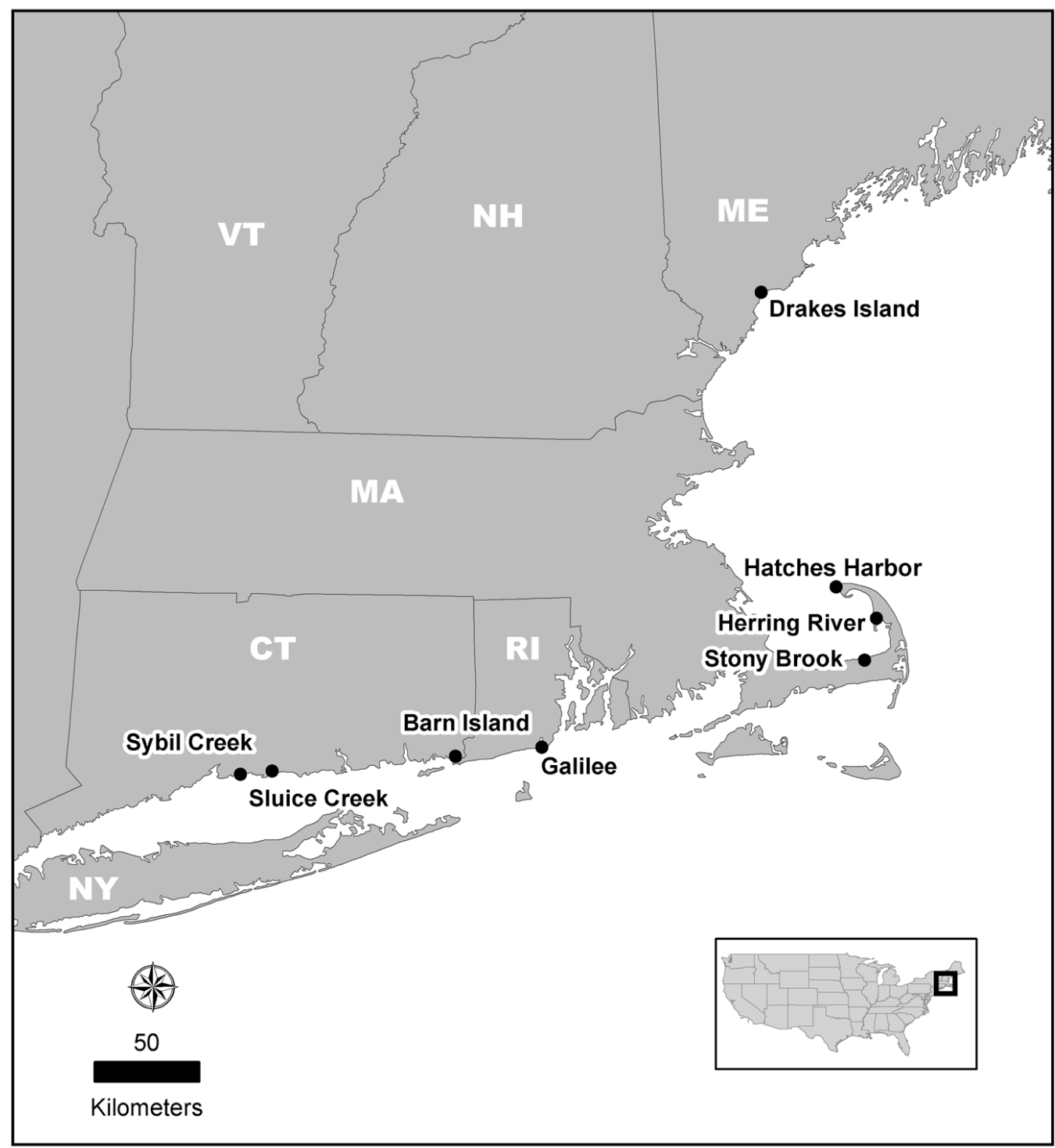


Figure 4-2. Proportion of gravid female fish in each marsh type, July 2011 (error bars: \pm standard deviation).

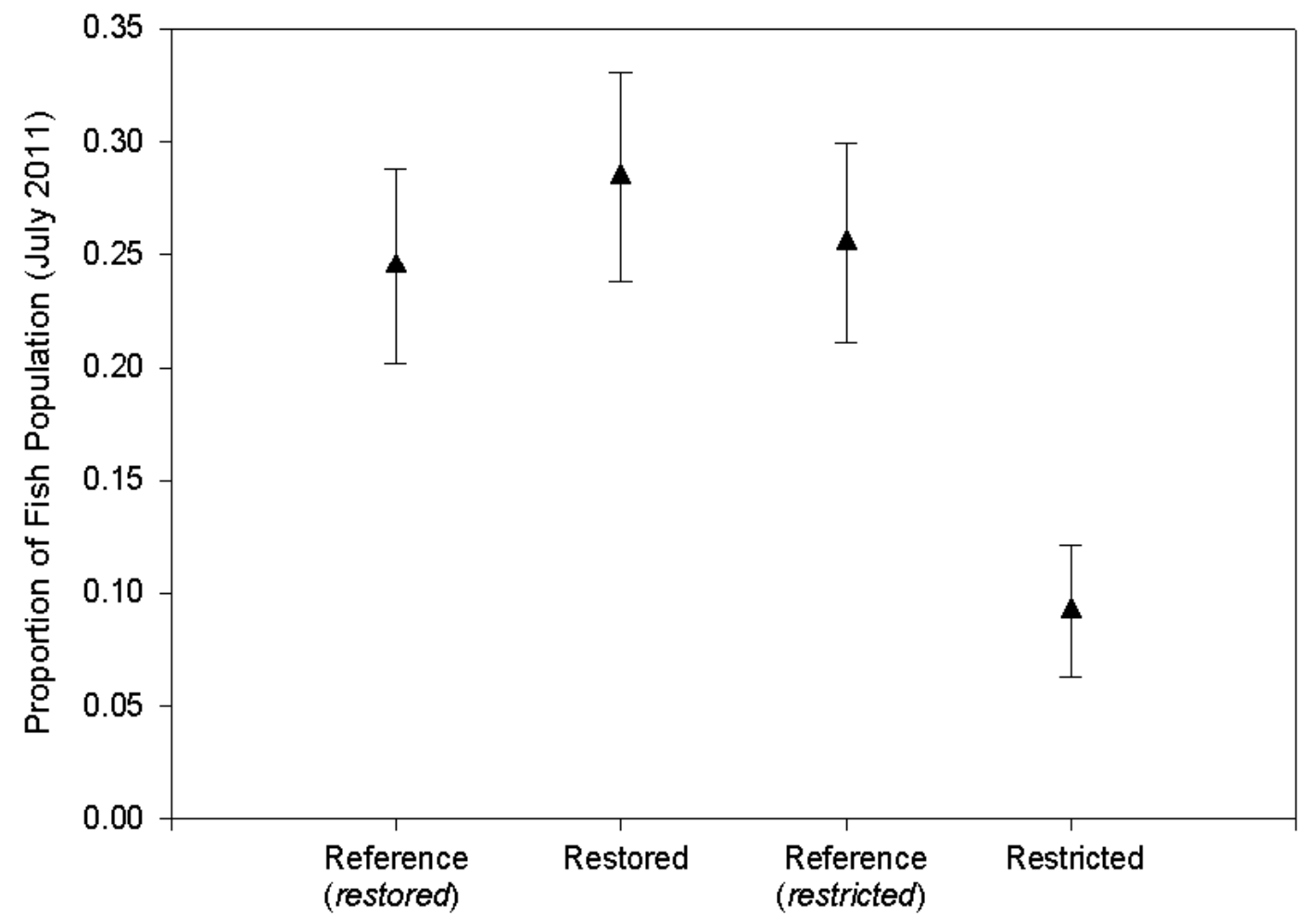

